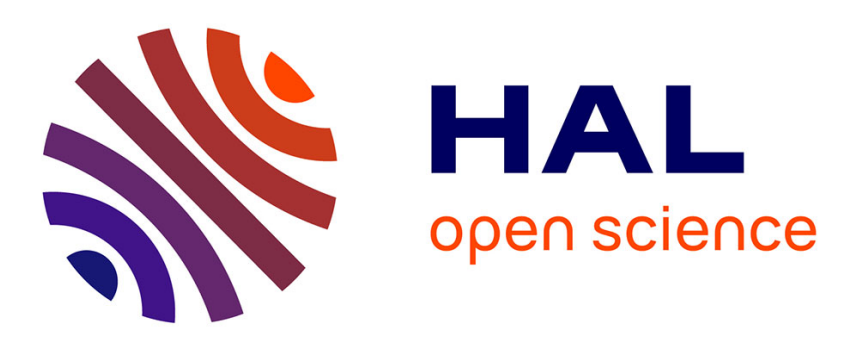

\title{
The Ginzburg-Landau functional with a discontinuous and rapidly oscillating pinning term. Part II: the non-zero degree case
}

\author{
Mickaël dos Santos
}

\section{- To cite this version:}

Mickaël dos Santos. The Ginzburg-Landau functional with a discontinuous and rapidly oscillating pinning term. Part II: the non-zero degree case. Indiana University Mathematics Journal, 2013, 62 (2), pp.551-641. hal-00587804v4

\section{HAL Id: hal-00587804 \\ https://hal.science/hal-00587804v4}

Submitted on 6 Nov 2011

HAL is a multi-disciplinary open access archive for the deposit and dissemination of scientific research documents, whether they are published or not. The documents may come from teaching and research institutions in France or abroad, or from public or private research centers.
L'archive ouverte pluridisciplinaire HAL, est destinée au dépôt et à la diffusion de documents scientifiques de niveau recherche, publiés ou non, émanant des établissements d'enseignement et de recherche français ou étrangers, des laboratoires publics ou privés. 


\title{
The Ginzburg-Landau functional with a discontinuous and rapidly oscillating pinning term. Part II: the non-zero degree case
}

\author{
Mickaël Dos SAnTos* \\ mickael.dos-santos@u-pec.fr
}

November 7, 2011

\begin{abstract}
We consider minimizers of a Ginzburg-Landau energy with a discontinuous and rapidly oscillating pinning term, subject to a Dirichlet boundary condition of degree $d>0$. The pinning term models an unbounded number of small impurities in the domain. We prove that for strongly type II superconductor with impurities, minimizers have exactly $d$ isolated zeros (vortices). These vortices are of degree 1 and pinned by the impurities. As in the standard case studied by Bethuel, Brezis and Hélein, the macroscopic location of vortices is governed by vortex/vortex and vortex/ boundary repelling effects. In some special cases we prove that their macroscopic location tends to minimize the renormalized energy of Bethuel-Brezis-Hélein. In addition, impurities affect the microscopic location of vortices. Our technics allows us to work with impurities having different size. In this situation we prove that vortices are pinned by the largest impurities.
\end{abstract}

\section{Contents}

1 Introduction 3

2 Main results 8

3 Shrinking holes for weighted Dirichlet functionals 11

3.1 Existence results . . . . . . . . . . . . . . . . . . . 11

3.1.1 Existence of minimal maps defined in a perforated domain . . . . . . 11

3.1.2 Existence of an optimal perforated domain . . . . . . . . . . . . . . 12

3.2 Dirichlet Vs Degree Conditions in a fixed perforated domain . . . . . . . . . 13

3.3 Optimal perforated domains for the degree conditions . . . . . . . . . 14

3.3.1 The case of the periodic pinning term . . . . . . . . . . 15

3.3.2 Sharper result in the periodic case with dilution . . . . . . . . 17

3.3.3 The case of a general pinning term with variable sizes of inclusions . 17

${ }^{*}$ Université Paris Est-Créteil, 61 avenue du Général de Gaulle, 94010 Créteil Cedex 
4 The pinned Ginzburg-Landau functional

4.1 Sharp Upper Bound, $\eta$-ellipticity and Uniform Convergence . . . . . . . . . 18

4.1.1 Sharp Upper Bound and an $\eta$-ellipticity result . . . . . . . . . . . . . 18

4.1.2 Uniform convergence of $\left|v_{\varepsilon}\right|$ outside $\omega_{\varepsilon} \ldots \ldots \ldots \ldots$

$4.2 \mathrm{Bad}$ discs $\ldots \ldots \ldots \ldots \ldots \ldots \ldots$

4.2.1 Construction and first properties of bad discs . . . . . . . . . 20

4.2 .2 Location and degree of bad discs . . . . . . . . . . . . . . . 24

$4.3 H_{\text {loc }}^{1}$-weak convergence . . . . . . . . . . . . . . . 24

4.3.1 The contribution of the modulus is bounded in the whole domain . . 25

4.3.2 We bound the energy in a fixed perforated domain . . . . . . . . 25

4.3.3 We establish the limiting equation . . . . . . . . . . 26

4.4 The small bad discs . . . . . . . . . . . . . . . . . . . . . . 27

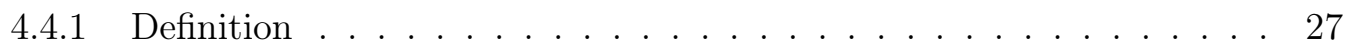

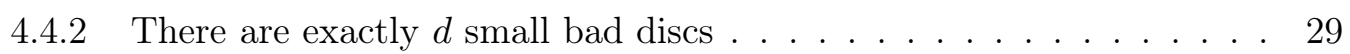

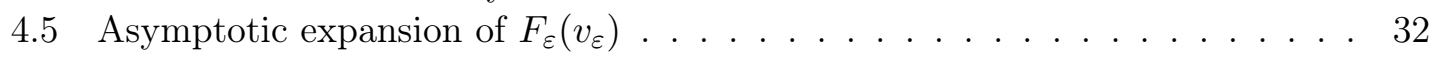

4.5.1 Statement of the main result and a corollary . . . . . . . . . . . . 32

4.5.2 Proof of Theorem 5. . . . . . . . . . . . . . 33

4.5.3 Proof of Proposition $40 \ldots \ldots \ldots \ldots \ldots$

4.6 Proof of Theorems 1, 2 , 3 and $4 \ldots \ldots \ldots \ldots \ldots$

A Proof of Proposition 8

B Proof of Proposition 9

C Proof of Proposition 10 39

C.1 The separation process . . . . . . . . . . . . . . . . . . . . . . . . . . . 39

C.2 The separation process gives a natural partition of $\Omega \ldots \ldots \ldots$. . . 41

C.3 Construction of test functions . . . . . . . . . . . . . . . . 41

C.4 Proof of Proposition 10 . . . . . . . . . . . . . . . . . . . . . . . . . . 46

D Proof of Proposition 12

D.1 Description of the special solution $U_{\varepsilon} \ldots \ldots \ldots \ldots \ldots$

D.2 Behavior of almost minimizers of $I_{\rho, \varepsilon} \ldots \ldots \ldots \ldots \ldots \ldots$

D.2.1 Useful results for the periodic situation . . . . . . . . . . . . . . 49

D.2.2 Lower bound on circles . . . . . . . . . . . . . . . . . . 52

D.3 Proof of the first part of Proposition $12 \ldots \ldots \ldots \ldots \ldots$

D.4 Proof of the second part of Proposition $12 \ldots \ldots \ldots \ldots \ldots$

D.5 Proof of the third part of Proposition $12 \ldots \ldots \ldots \ldots$

E Proof of Propositions 16 and 17

E.1 An important effect of the dilution of inclusions . . . . . . . . . . 60

E.2 Proof of Proposition 16 . . . . . . . . . . . . . . . . . . . . . . . 61

E.3 Proof of Proposition 17 . . . . . . . . . . . . . . . . . . . . . . . 66

F Proof of Proposition 32 


\section{Introduction}

In this article we let $\Omega \subset \mathbb{R}^{2}$ be a smooth simply connected domain and let $a_{\varepsilon}: \Omega \rightarrow$ $\{b, 1\}, b \in(0,1)$ be a measurable function. We associate to $a_{\varepsilon}$ the pinned Ginzburg-Landau energy

$$
E_{\varepsilon}(u)=\frac{1}{2} \int_{\Omega}\left\{|\nabla u(x)|^{2}+\frac{1}{2 \varepsilon^{2}}\left(a_{\varepsilon}(x)^{2}-|u(x)|^{2}\right)^{2}\right\} \mathrm{d} x .
$$

Here, $u: \Omega \rightarrow \mathbb{C}$ is in the Sobolev space $H^{1}(\Omega, \mathbb{C})$ and $\varepsilon>0$ is the inverse of the GinzburgLandau parameter.

Our goal is to consider a discontinuous and rapidly oscillating pinning term (the pinning term is $\left.a_{\varepsilon}: \Omega \rightarrow\{b, 1\}\right)$. Our pinning term is periodic with respect to a $\delta \times \delta$-grid with $\delta=\delta(\varepsilon) \rightarrow 0$ as $\varepsilon \rightarrow 0$ (in some cases we drop the periodic hypothesis).

We are interested in the minimization of (1.1) in $H^{1}(\Omega, \mathbb{C})$ subject to a Dirichlet boundary condition: we fix $g \in C^{\infty}\left(\partial \Omega, \mathbb{S}^{1}\right)$ and thus the set of the test functions is

$$
H_{g}^{1}:=\left\{u \in H^{1}(\Omega, \mathbb{C}) \mid \operatorname{tr}_{\partial \Omega} u=g\right\} .
$$

The situation where $d=\operatorname{deg}_{\partial \Omega}(g)=0$ was studied in detail in [12]. The non zero degree case $\left(d=\operatorname{deg}_{\partial \Omega}(g)>0\right)$ is the purpose of the present article. Recall that for $\Gamma \subset \mathbb{R}^{2}$ a Jordan curve and $g \in H^{1 / 2}\left(\Gamma, \mathbb{S}^{1}\right.$ ), the degree (winding number) of $g$ is defined as

$$
\operatorname{deg}_{\Gamma}(g):=\frac{1}{2 \pi} \int_{\Gamma} g \times \partial_{\tau} g \mathrm{~d} \tau
$$

Here " $\times$ " stands for the vectorial product in $\mathbb{C}$, i.e. $z_{1} \times z_{2}=\operatorname{Im}\left(\overline{z_{1}} z_{2}\right), z_{1}, z_{2} \in \mathbb{C}, \tau$ is the direct unit tangent vector of $\Gamma\left(\tau=\nu^{\perp}\right.$ where $\nu$ is the outward normal unit vector of $\operatorname{int}(\Gamma)$, the bounded open set whose boundary is $\Gamma)$ and $\partial_{\tau}$ is the tangential derivative on $\Gamma$.

This energy is a simplification of the full Ginzburg-Landau energy (see Eq. (1.2) below) whose minimizers model the state of a Type II superconductor (the parameter $\varepsilon$ corresponds to a material parameter, this parameter is small for Type II superconductor) [23], [20]. The pinning term allows to model a heterogenous superconductor (see [15] or Introduction of [11]).

Physical informations which can be obtained with the simplification of the full GinzburgLandau energy are quantization and location of zeros of minimizers. Their zeros represent the centers of small areas where the superconductivity is destroyed. These areas are called vorticity defects. Here the superconductor is a cylinder whose cross section is $\Omega$ and the vorticity defects (under some special conditions) takes the form of small wires parallel to the superconductor [23, 20].

Before going further, let us summarize two previous works in related directions [17, [1]. In these works, the role of the pinning term is identified: its points of minimum attract the vorticity defects.

In [17, Lassoued and Mironescu considered the case where $a_{\varepsilon} \equiv a$. Here, the pinning term $a=\left\{\begin{array}{ll}b & \text { in } \omega \\ 1 & \text { in } \Omega \backslash \omega\end{array}, 0<b<1\right.$, and $\omega$ is a smooth inner domain of $\Omega$. These authors proved that the vorticity defects are quantified by $\operatorname{deg}_{\partial \Omega}(g)$, localized in $\omega$ and that their position is governed by a renormalized energy (in the spirit of 44).

In 11, Aftalion, Sandier and Serfaty considered a smooth and $\varepsilon$-dependent pinning term $a_{\varepsilon}$. Their study allows to consider the case where the pinning term has fast oscillations: it is a perturbation of a fixed smooth function $\tilde{b}: \Omega \rightarrow[b, 1]$ s.t. $a_{\varepsilon} \geq \tilde{b}$. 
In contrast with [17], [1] is dedicated to the study of a full Ginzburg-Landau energy $G L_{\varepsilon}$ with the pinning term $a_{\varepsilon}$

$$
G L_{\varepsilon}(u, A)=\frac{1}{2} \int_{\Omega}\left\{\left|\operatorname{curl} A-h_{\mathrm{ex}}\right|^{2}+|(\nabla-i A) u|^{2}+\frac{1}{2 \varepsilon^{2}}\left(a_{\varepsilon}^{2}-|u|^{2}\right)^{2}\right\} .
$$

We denoted by $A \in \mathbb{R}^{2}$ the electromagnetic vector potential of the induced field and by $h_{\mathrm{ex}} \gg 1$ the intensity of the applied magnetic field (see [20] for more details).

They considered the following hypotheses on $a_{\varepsilon}, \tilde{b}$ :

- $\left|\nabla a_{\varepsilon}\right| \leq C h_{\mathrm{ex}}$

- there is $\sigma_{\varepsilon} \in \mathbb{R}$ s.t. $\sigma_{\varepsilon}=o\left((\ln |\ln \varepsilon|)^{-1 / 2}\right)$ and for all $x \in \Omega$, we have

$$
\min _{B\left(x, \sigma_{\varepsilon}\right)}\left\{a_{\varepsilon}-\tilde{b}\right\}=0
$$

In the study of the full Ginzburg-Landau functional without pinning term $G L_{\varepsilon}^{0}\left(G L_{\varepsilon}^{0}\right.$ is obtained from (1.2) by taking $a_{\varepsilon} \equiv 1$ ), the vorticity defects appear for large apply magnetic field. They are characterized by two facts: the presence of isolated zeros $x_{i}$ of a map $u$ with a non zero degree around small circles centered in $x_{i}$ and the existence of a magnetic field inside the domain $\left(\operatorname{curl}(A) \simeq h_{\mathrm{ex}}\right.$ inside small discs). The nature of the superconductivity makes that both facts appear together. Assume that the intensity of the applied field $h_{\mathrm{ex}}$ depends on $0<\varepsilon<1$ and that $h_{\mathrm{ex}} /|\ln \varepsilon| \rightarrow \Lambda \in \mathbb{R}_{+}^{*}$. For the full Ginzburg-Landau energy without pinning term $G L_{\varepsilon}^{0}$, it is well known (see e.g. [20]) that there is an inner domain $\omega_{\Lambda}$ (non decreasing w.r.t. $\Lambda$ ) s.t., when $\varepsilon \rightarrow 0$, the vorticity defects are "uniformly located" by $\omega_{\Lambda}$ (in this situation the number of vortices is unbounded).

In [1] (study of a full Ginzburg-Landau functional with a pinning term), the authors proved the existence of $\omega_{\Lambda}$, an inner set of $\Omega$, where the penetration of the magnetic field is located. In contrast with the situation without pinning term, the presence of $a_{\varepsilon}$ makes that, in general, the vortices are not uniformly located in $\omega_{\Lambda}$. Although in the proofs of the main results of [1, the minimal points of $\tilde{b}$ seem play the role of a pinning site, this fact is not proved. They expect that the most favorable pinning sites should be close to the minima of $\tilde{b}: \omega_{\Lambda}$ should be located close to the points of minimum of $\tilde{b}$.

One of our goals is to prove that the minimum points of a rapidly oscillating and discontinuous pinning term attract the vorticity defects.

Before going further, we construct our (periodic) pinning term $a_{\varepsilon}$.

Construction 1. The periodic pinning term

Consider

- $\delta=\delta(\varepsilon) \in(0,1), \lambda=\lambda(\varepsilon) \in(0,1]$

- $\omega \subset Y=(-1 / 2,1 / 2)^{2}$ be a smooth bounded and simply connected open set s.t. $(0,0) \in$ $\omega$ and $\bar{\omega} \subset Y$ (here $Y$ is the unit cell).

For $k, l \in \mathbb{Z}$ we denote

$$
\begin{gathered}
Y_{k, l}^{\delta}:=\delta \cdot Y+(\delta k, \delta l), \quad \Omega_{\delta}^{\text {incl }}=\bigcup_{Y_{k, l}^{\delta} \subset \Omega} \overline{Y_{k, l}^{\delta},} \quad \omega^{\lambda}=\lambda \cdot \omega, \\
\omega_{\text {per }}^{\lambda}=\bigcup_{(k, l) \in \mathbb{Z}^{2}}\left\{\omega^{\lambda}+(k, l)\right\} \text { and } \omega_{\varepsilon}=\bigcup_{\substack{(k, l) \in \mathbb{Z}^{2} \text { s.t. } \\
Y_{k, l}^{\delta} \subset \Omega}}\left\{\delta \cdot \omega^{\lambda}+(\delta k, \delta l)\right\} .
\end{gathered}
$$


For $b \in(0,1)$, we define

$$
\begin{aligned}
& a^{\lambda}: \mathbb{R}^{2} \rightarrow \quad\{b, 1\} \quad a_{\varepsilon}: \mathbb{R}^{2} \rightarrow \quad\{b, 1\} \\
& x \mapsto\left\{\begin{array} { l l } 
{ b } & { \text { if } x \in \omega _ { \text { per } } ^ { \lambda } } \\
{ 1 } & { \text { otherwise } }
\end{array} \text { and } \quad x \mapsto \left\{\begin{array}{ll}
b & \text { if } x \in \omega_{\varepsilon} \\
1 & \text { otherwise }
\end{array} .\right.\right.
\end{aligned}
$$

The values of the periodic pinning term are represented Figure 1. The connected components of $\left\{a_{\varepsilon}=b\right\}=\omega_{\varepsilon}$ are called inclusions or impurities.

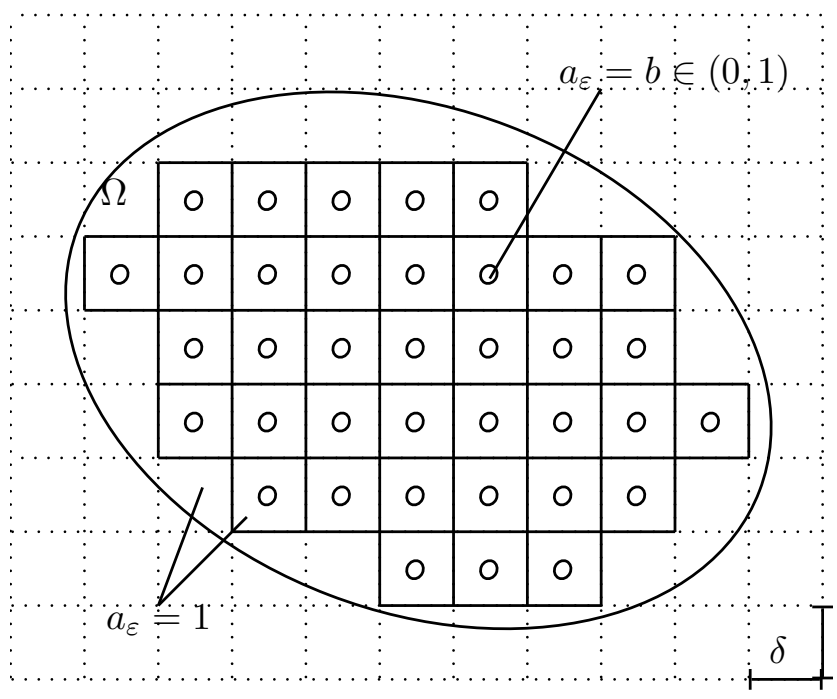

(a) The pining term is periodic on a $\delta \times \delta$-grid

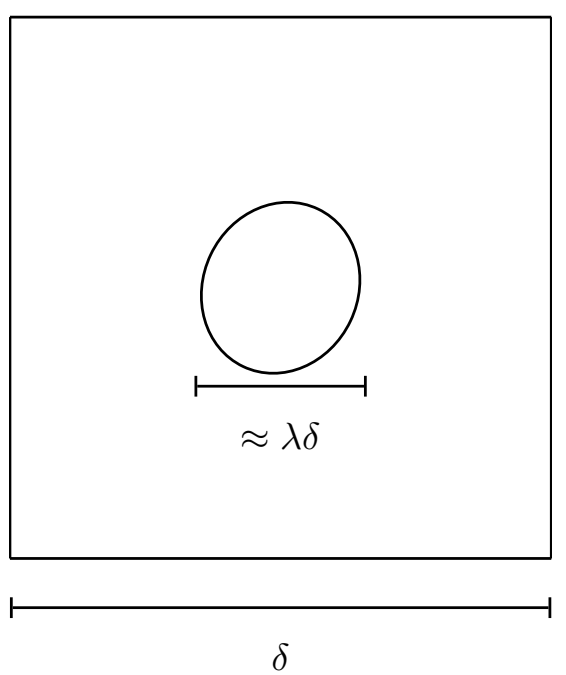

(b) The parameter $\lambda$ controls the size of an inclusion in the cell

Figure 1: The periodic pinning term

In the rest of this article $\lambda=\lambda(\varepsilon)$ and $\delta=\delta(\varepsilon)$ are functions of $\varepsilon$. We assume that $\delta \rightarrow 0$ as $\varepsilon \rightarrow 0$. In addition, we assume that either $\lambda \equiv 1$, or $\lambda \rightarrow 0$ as $\varepsilon \rightarrow 0$. The case $\lambda \rightarrow 0$ is the diluted case.

We make the (technical) assumption

$$
\lim _{\varepsilon} \frac{|\ln (\lambda \delta)|^{3}}{|\ln \varepsilon|}=0
$$

Remark 2. - This is slightly more restrictive than asking that $\lambda \delta \gg \varepsilon^{\alpha}$ for all $\alpha \in(0,1)$.

- Hypothesis (1.3) is technical, a more natural hypothesis should be $\lambda \delta \gg \varepsilon$ or $\lambda \delta \gg \varepsilon^{\alpha}$ for some $\alpha \in(0,1)$.

- In [1] and in the situation where we have a bounded number of zeros (the applied magnetic field is not too large), the smooth pinning term $a_{\varepsilon}^{0}$ satisfies the condition $\left|\nabla a_{\varepsilon}^{0}\right| \leq C|\ln \varepsilon|$. In order to compare this assumption with (1.3), we may consider a regularization of our pinning term by a mollifier $\rho_{t}(x)=t^{-2} \rho(x / t)$. A suitable scale $t$ to have a complete view of the variations of $a_{\varepsilon}$ is $t=\lambda \delta$. Thus, $\left|\nabla\left(\rho_{\lambda \delta} * a_{\varepsilon}\right)\right|$ is of order $\frac{1}{\lambda \delta}$. Consequently, the condition (1.3) allows to consider a more rapidly oscillating than 
the condition in [1]. Indeed, we have $\ln \left|\nabla a_{\varepsilon}^{0}\right| \lesssim \ln |\ln \varepsilon|$ and on the other hand (1.3) is equivalent to $\ln \left|\nabla\left(\rho_{\lambda \delta} * a_{\varepsilon}\right)\right| \lesssim|\ln (\lambda \delta)|=o\left(|\ln \varepsilon|^{1 / 3}\right)$.

The goal of this article is to study the minimizers of

$$
E_{\varepsilon}(u)=\frac{1}{2} \int_{\Omega}\left\{|\nabla u|^{2}+\frac{1}{2 \varepsilon^{2}}\left(a_{\varepsilon}^{2}-|u|^{2}\right)^{2}\right\}, u \in H_{g}^{1}
$$

in the asymptotic $\varepsilon \rightarrow 0$. A standard method (initiated in [17]) consists in decoupling $E_{\varepsilon}$ into a sum of two functionals. The key tool in this method is $U_{\varepsilon}$ the unique global minimizer of $E_{\varepsilon}$ in $H_{1}^{1}$ (see [17]). Clearly, $U_{\varepsilon}$ satisfies

$$
\left\{\begin{array}{ll}
-\Delta U_{\varepsilon}=\frac{1}{\varepsilon^{2}} U_{\varepsilon}\left(a_{\varepsilon}^{2}-U_{\varepsilon}^{2}\right) & \text { in } \Omega \\
U_{\varepsilon}=1 & \text { on } \partial \Omega
\end{array} .\right.
$$

From the uniqueness of $U_{\varepsilon}$, by construction of a test function, it is easy to get that $b \leq$ $U_{\varepsilon} \leq 1$.

This special solution may be seen as a regularization of $a_{\varepsilon}$. For example, one may easily prove that $U_{\varepsilon}$ is exponentially close to $a_{\varepsilon}$ far away from $\partial \omega_{\varepsilon}$ (a more complete description of $U_{\varepsilon}$ is done Appendix (D.1). Namely, we have

Proposition 3. There are $C, \alpha>0$ independent of $\varepsilon, R>0$ s.t.

$$
\begin{gathered}
\left|a_{\varepsilon}-U_{\varepsilon}\right| \leq C \mathrm{e}^{-\frac{\alpha R}{\varepsilon}} \text { in } V_{R}:=\left\{x \in \Omega \mid \operatorname{dist}\left(x, \partial \omega_{\varepsilon}\right) \geq R\right\}, \\
\left|\nabla U_{\varepsilon}\right| \leq \frac{C \mathrm{e}^{-\frac{\alpha R}{\varepsilon}}}{\varepsilon} \text { in } W_{R}:=\left\{x \in \Omega \mid \operatorname{dist}\left(x, \partial \omega_{\varepsilon}\right), \operatorname{dist}(x, \partial \Omega) \geq R\right\} .
\end{gathered}
$$

A similar result was proved in [13] (Proposition 2). The above proposition yields by the same arguments.

As in [17], we define

$$
F_{\varepsilon}(v)=\frac{1}{2} \int_{\Omega}\left\{U_{\varepsilon}^{2}|\nabla v|^{2}+\frac{1}{2 \varepsilon^{2}} U_{\varepsilon}^{4}\left(1-|v|^{2}\right)^{2}\right\} .
$$

Then we have for all $v \in H_{g}^{1}$, (see [17])

$$
E_{\varepsilon}\left(U_{\varepsilon} v\right)=E_{\varepsilon}\left(U_{\varepsilon}\right)+F_{\varepsilon}(v) .
$$

Therefore, $u_{\varepsilon}$ is a minimizer of $E_{\varepsilon}$ if and only if $u_{\varepsilon}=U_{\varepsilon} v_{\varepsilon}$ where $v_{\varepsilon}$ is a minimizer of $F_{\varepsilon}$ in $H_{g}^{1}$. Consequently, the study of a minimizer $u_{\varepsilon}=U_{\varepsilon} v_{\varepsilon}$ of $E_{\varepsilon}$ in $H_{g}^{1}$ (location of zeros and asymptotics) can be performed by combining the asymptotic of $U_{\varepsilon}$ with one of $v_{\varepsilon}$.

Our main result is the following

Theorem 1. Assume that $\lambda, \delta$ satisfy (1.3) and that $\lambda \rightarrow 0$.

Quantization. There are $\varepsilon_{0}>0, c>0$ and $\eta_{0}>0$ s.t. for $0<\varepsilon<\varepsilon_{0}$ :

1. $v_{\varepsilon}$ has exactly $d$ zeros $x_{1}^{\varepsilon}, \ldots, x_{d}^{\varepsilon}$,

2. $B\left(x_{i}^{\varepsilon}, c \lambda \delta\right) \subset \omega_{\varepsilon}$, 
3. for $\rho=\rho(\varepsilon) \downarrow 0$ s.t. $|\ln \rho| /|\ln \varepsilon| \rightarrow 0$, there is $C>0$ independent of $\varepsilon$ satisfying

$$
\left|v_{\varepsilon}\right| \geq 1-C \sqrt{\frac{|\ln \rho|}{|\ln \varepsilon|}} \text { in } \Omega \backslash \cup \overline{B\left(x_{i}^{\varepsilon}, \rho\right)} \text {, }
$$

4. for $\varepsilon<\varepsilon_{0}$

- There are two repulsive effects: $\left|x_{i}^{\varepsilon}-x_{j}^{\varepsilon}\right| \geq \eta_{0}$ for $i \neq j$ and $\operatorname{dist}\left(x_{i}^{\varepsilon}, \partial \Omega\right) \geq \eta_{0}$;

- $\operatorname{deg}_{\partial B\left(x_{i}^{\varepsilon}, \delta\right)}\left(v_{\varepsilon}\right)=1$.

Location.

- The macroscopic location of the zeros tends to minimize the renormalized energy of Bethuel-Brezis-Hélein $W_{g}:\left\{\left\{x_{1}, \ldots, x_{d}\right\} \subset \Omega \mid x_{i} \neq x_{j}\right.$ for $\left.i \neq j\right\} \rightarrow \mathbb{R}$ (defined in [4], Chapter I Eq. (47)):

$$
\limsup W_{g}\left(x_{1}^{\varepsilon}, \ldots, x_{d}^{\varepsilon}\right)=\min _{\substack{a_{1}, \ldots, a_{d} \in \Omega \\ a_{i} \neq a_{j}}} W_{g}\left(a_{1}, \ldots, a_{d}\right)
$$

- The microscopic location of the zeros inside $\omega_{\varepsilon}$ tends to depend only on $\omega$ and $b$ :

- since $x_{i}^{\varepsilon} \in \omega_{\varepsilon}$, we have $x_{i}^{\varepsilon}=\left(k_{\varepsilon} \delta, l_{\varepsilon} \delta\right)+\lambda \delta y_{i}^{\varepsilon}$ with $k_{\varepsilon}, l_{\varepsilon} \in \mathbb{Z}$ and $y_{i}^{\varepsilon} \in \omega$;

- for $\varepsilon_{n} \downarrow 0$ s.t. $y_{i}^{\varepsilon_{n}} \rightarrow \hat{\hat{a}}_{i}$, we have $\hat{\hat{a}}_{i} \in \omega$ which minimizes a renormalized energy $\tilde{W}_{1}: \omega \rightarrow \mathbb{R}$ (given in [13] Eq. (90)) which depends only on $\omega$ and $b \in(0,1)$.

Remark 4. 1. The renormalized energy defined in [4]

$$
W_{g}:\left\{\left\{x_{1}, \ldots, x_{d}\right\} \subset \Omega \mid x_{i} \neq x_{j} \text { for } i \neq j\right\} \rightarrow \mathbb{R}
$$

governs the location of the zeros in the situation where $a_{\varepsilon} \equiv 1$ (homogenous case): the zeros tend to minimize $W_{g}$. In [4] (Chapter 1), the authors defined a renormalized energy in a more general setting

$$
W_{g}^{\mathrm{BBH}}:\left\{\begin{array}{l|l}
\left\{\left(x_{1}, d_{1}\right), \ldots,\left(x_{N}, d_{N}\right)\right\} & \begin{array}{c}
x_{i} \in \Omega, x_{i} \neq x_{j} \text { for } i \neq j \\
d_{i} \in \mathbb{Z} \text { is s.t. } \sum_{i=1}^{N} d_{i}=d
\end{array}
\end{array}\right\} \rightarrow \mathbb{R} .
$$

Here $W_{g}\left(x_{1}, \ldots, x_{d}\right)=W_{g}^{\mathrm{BBH}}\left(\left\{\left(x_{1}, 1\right), \ldots,\left(x_{d}, 1\right)\right\}\right)$, i.e., in this article we will consider only the renormalized energy with the degrees equal 1 and thus we do not specify the degrees in its notation.

2. From smoothness of $W_{g}$ (see [4] and [10]), Location part of Theorem 1 implies that up to pass to a subsequence, the zeros converge to a minimizer of $W_{g}$.

3. This macroscopic location is strongly correlated with the Dirichlet boundary condition $g \in C^{\infty}\left(\partial \Omega, \mathbb{S}^{1}\right)$.

4. The result about the macroscopic position of the periodic and diluted pinning term may be sum up as: the macroscopic position of the zeros tends to be the same than in the homogenous case $\left(a_{\varepsilon} \equiv 1\right)$.

5. The microscopic location of the zeros (position inside an inclusion) is independent of the boundary condition. For example, in the situation $\omega=B\left(0, r_{0}\right)$, i.e., the inclusions are discs, this location should be the center of the inclusion. This fact is not proved yet.

6. In Assertion 4. of Quantization part, $\operatorname{deg}_{\partial B\left(x_{i}^{\varepsilon}, \delta\right)}\left(v_{\varepsilon}\right)=\operatorname{deg}_{\partial B\left(x_{i}^{\varepsilon}, \delta\right)}\left(v_{\varepsilon} /\left|v_{\varepsilon}\right|\right)$. 


\section{Main results}

We present in this section several extensions of the above result dropping either the dilution of the inclusion $(\lambda \equiv 1$ instead of $\lambda \rightarrow 0)$ or the periodic structure. The main results of this section are obtained under the condition: $\lambda \delta$ satisfies (1.3).

Our sharper results are shared into four theorems:

- The first theorem (Theorem 2) gives informations on the zeros of minimizers $u_{\varepsilon}, v_{\varepsilon}$ (quantization and location).

- The second theorem (Theorem 3) establishes the asymptotic behavior of $v_{\varepsilon}$.

- The third theorem (Theorem 4) establishes, under the additional hypothesis $\lambda \rightarrow 0$, that the microscopic position of the zeros is independent of the boundary condition $g$.

- The last theorem (Theorem 5) gives an expansion of $F_{\varepsilon}\left(v_{\varepsilon}\right)$.

The technics developed in this paper allows to consider either the case $\lambda \rightarrow 0$ or $\lambda \equiv 1$. The results in the diluted case are more precise. One may drop the periodic structure for the pinning term and consider impurities (the connected components of $\omega_{\varepsilon}=\left\{a_{\varepsilon}=b\right\}$ ) with different sizes (adding the hypothesis $\lambda \rightarrow 0$ ).

More precisely we may consider the pinning term defined as follow:

Construction 5. The general diluted pinning term

- Fix $P \in \mathbb{N}^{*}, j \in\{1, \ldots, P\}$ and $1>\varepsilon>0$. We consider $M_{j}^{\varepsilon} \in \mathbb{N}$ and

$$
\mathcal{M}_{j}^{\varepsilon}= \begin{cases}\emptyset & \text { if } M_{j}^{\varepsilon}=0 \\ \left\{1, \ldots, M_{j}^{\varepsilon}\right\} & \text { if } M_{j}^{\varepsilon} \in \mathbb{N}^{*}\end{cases}
$$

- The sets $\mathcal{M}_{j}^{\varepsilon}$ 's are s.t. (for sufficiently small $\varepsilon$ ) one may fix $y_{i, j}^{\varepsilon} \in \Omega$ s.t. for $(i, j) \neq\left(i^{\prime}, j^{\prime}\right)$, $i \in \mathcal{M}_{j}^{\varepsilon}, i^{\prime} \in \mathcal{M}_{j^{\prime}}^{\varepsilon}$ we have

$$
\left|y_{i, j}^{\varepsilon}-y_{i^{\prime}, j^{\prime}}^{\varepsilon}\right| \geq \delta^{j}+\delta^{j^{\prime}} \text { and } \operatorname{dist}\left(y_{i, j}^{\varepsilon}, \partial \Omega\right) \geq \delta^{j} .
$$

We denote $\widehat{\mathcal{M}}_{j}^{\varepsilon}:=\left\{y_{i, j}^{\varepsilon} \mid i \in \mathcal{M}_{j}^{\varepsilon}\right\}$.

For sake of simplicity, we assume that there is $\eta>0$ s.t. for small $\varepsilon$, we have $M_{1}^{\varepsilon} \geq d=$ $\operatorname{deg}_{\partial \Omega}(g)$ and

$$
\min \left\{\min _{i=1, \ldots, d} \operatorname{dist}\left(y_{i, 1}^{\varepsilon}, \partial \Omega\right), \min _{\substack{i, i^{\prime}=1, \ldots, d \\ i \neq i^{\prime}}}\left|y_{i, 1}^{\varepsilon}-y_{i^{\prime}, 1}^{\varepsilon}\right|\right\} \geq \eta
$$

- We now define the domain which models the impurities:

$$
\omega_{\varepsilon}=\bigcup_{j=1}^{P} \bigcup_{i \in \mathcal{M}_{j}^{\varepsilon}}\left\{y_{i, j}^{\varepsilon}+\delta^{j} \cdot \omega^{\lambda}\right\}, \omega^{\lambda}=\lambda \cdot \omega
$$




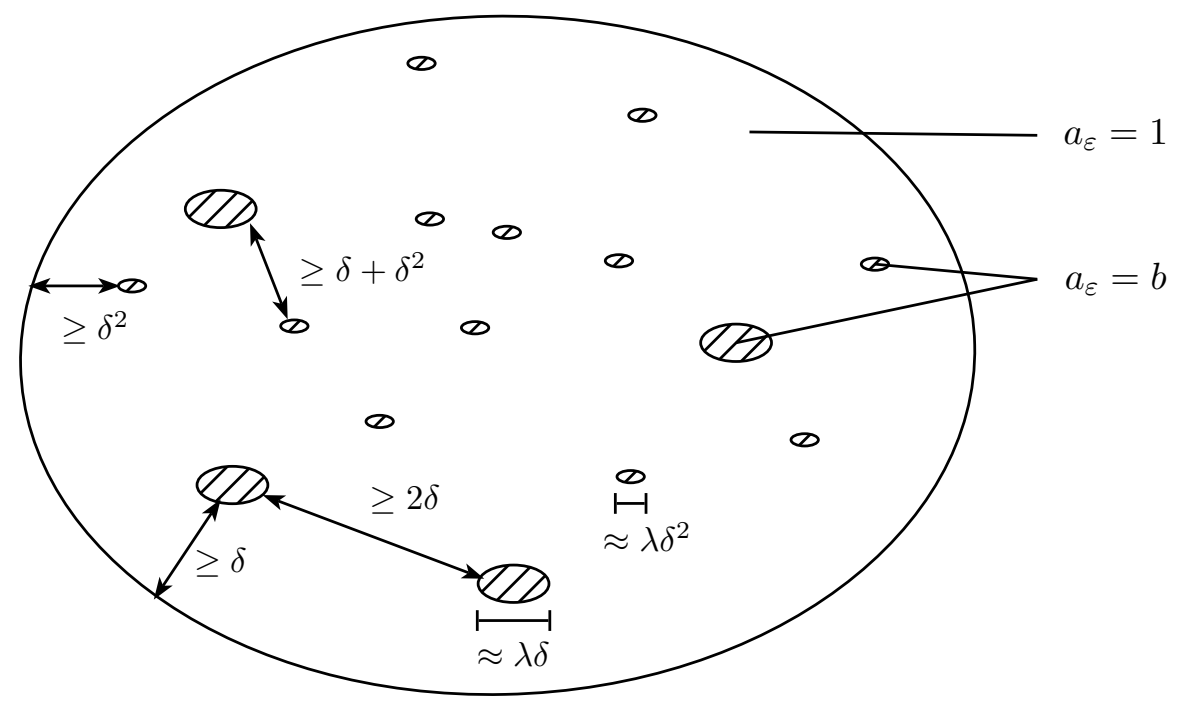

Figure 2: Representation of the general diluted pinning term with $P=2$

The pinning term is

$$
\begin{aligned}
a_{\varepsilon}: \mathbb{R}^{2} & \rightarrow \begin{cases}\{b, 1\} \\
1 & \text { if } x \notin \omega_{\varepsilon} \\
b & \text { if } x \in \omega_{\varepsilon}\end{cases}
\end{aligned}
$$

The values of the pinning term are represented Figure 2 .

Our main results are

Theorem 2. Assume that $\lambda, \delta$ satisfy (1.3) and if the pinning term is not periodic (represented Figure 2) then we assume also that $\lambda \rightarrow 0$.

There is $\varepsilon_{0}>0$ s.t.:

1. for $0<\varepsilon<\varepsilon_{0}$, $v_{\varepsilon}$ has exactly $d$ zeros $x_{1}^{\varepsilon}, \ldots, x_{d}^{\varepsilon}$,

2. there are $c>0$ and $\eta_{0}>0$ s.t. for $\varepsilon<\varepsilon_{0}, B\left(x_{i}^{\varepsilon}, c \lambda \delta\right) \subset \omega_{\varepsilon}$ and

$$
\min _{i}\left\{\min _{j \neq i}\left|x_{i}^{\varepsilon}-x_{j}^{\varepsilon}\right|, \operatorname{dist}\left(x_{i}^{\varepsilon}, \partial \Omega\right)\right\} \geq \eta_{0} .
$$

In particular, if the pinning term is not periodic, then the zeros are trapped by the largest inclusions (those of size $\lambda \delta$ ).

3. for $\rho=\rho(\varepsilon) \downarrow 0$ s.t. $|\ln \rho| /|\ln \varepsilon| \rightarrow 0$, we have for $\varepsilon<\varepsilon_{0}$,

$$
\left|v_{\varepsilon}\right| \geq 1-C \sqrt{\frac{|\ln \rho|}{|\ln \varepsilon|}} \text { in } \Omega \backslash \cup \overline{B\left(x_{i}^{\varepsilon}, \rho\right)} .
$$

Here $C$ is independent of $\varepsilon$.

4. for $\varepsilon<\varepsilon_{0}, \operatorname{deg}_{\partial B\left(x_{i}^{\varepsilon}, \delta\right)}\left(v_{\varepsilon}\right)=1$. 
Remark 6. Hypothesis (2.2) is used to simplify the statements. Without this hypothesis, some of the results are subject to technical considerations on $\delta, \lambda, b \ldots$ For example if we consider the pinning term $a_{\varepsilon}$ defined in $\Omega=B(0,2)$ by

$$
\begin{aligned}
a_{\varepsilon}: B(0,2) & \rightarrow\{b, 1\} \\
x & \mapsto \begin{cases}b & \text { if } x \in B(0, \lambda \delta) \cup B\left(1, \lambda \delta^{2}\right), \\
1 & \text { otherwise }\end{cases}
\end{aligned}
$$

and $g \in C^{\infty}\left(\partial \Omega, \mathbb{S}^{1}\right)$ s.t. $\operatorname{deg}_{\partial \Omega}(g)=2$, then Hypothesis (2.2) is not satisfied. In this situation, we may prove that, for sufficiently small $\varepsilon, v_{\varepsilon}$ has exactly two zeros and if $2\left(1-2 b^{2}\right)|\ln \lambda|+\left(1-3 b^{2}\right)|\ln \delta| \rightarrow+\infty($ resp. $-\infty)$, then the zeroes are in $B(0, \lambda \delta)$ (resp. there is one zero inside $B(0, \lambda \delta)$ and one zero inside $\left.B\left(1, \lambda \delta^{2}\right)\right)$.

Theorem 3. Assume that $\lambda, \delta$ satisfy (1.3) and if the pinning term is not periodic (represented Figure 2) then we assume also that $\lambda \rightarrow 0$.

Let $\varepsilon_{n} \downarrow 0$, up to a subsequence, we have the existence of $a_{1}, \ldots, a_{d} \in \Omega, d$ distinct points s.t. $x_{i}^{\varepsilon_{n}} \rightarrow a_{i}$ and

$$
\left|v_{\varepsilon_{n}}\right| \rightarrow 1 \text { and } v_{\varepsilon_{n}} \rightarrow v_{*} \text { in } H_{\mathrm{loc}}^{1}\left(\bar{\Omega} \backslash\left\{a_{1}, \ldots, a_{d}\right\}, \mathbb{S}^{1}\right)
$$

where $v_{*}$ solves

$$
\left\{\begin{array}{ll}
-\operatorname{div}\left(\mathcal{A} \nabla v_{*}\right)=\left(\mathcal{A} \nabla v_{*} \cdot \nabla v_{*}\right) v_{*} & \text { in } \Omega \backslash\left\{a_{1}, \ldots, a_{d}\right\} \\
v_{*}=g & \text { on } \partial \Omega
\end{array} .\right.
$$

Here $\mathcal{A}$ is the homogenized matrix of $a^{2}\left(\frac{\cdot}{\delta}\right) \operatorname{Id}_{\mathbb{R}^{2}}$ if $\lambda \equiv 1$ and $\mathcal{A}=\operatorname{Id}_{\mathbb{R}^{2}}$ if $\lambda \rightarrow 0$.

In addition, for each $M>0, v_{\varepsilon, i}^{\prime}(\cdot)=v_{\varepsilon}\left(x_{i}^{\varepsilon}+\frac{\varepsilon}{b}\right)$ converges, up to a subsequence, in $C^{1}(B(0, M))$ to $f(|x|) \frac{x}{|x|} \mathrm{e}^{\imath \theta_{i}}$ where $f: \mathbb{R}^{+} \rightarrow \mathbb{R}^{+}$is the universal function defined in [19] and $\theta_{i} \in \mathbb{R}$.

Theorem 4. Assume, in addition to the hypotheses of Theorem [3, that $\lambda \rightarrow 0$.

Let $[x]=\left[\left(x_{1}, x_{2}\right)\right]=\left(\left[x_{1}\right],\left[x_{2}\right]\right) \in \mathbb{Z}^{2}$ be the vectorial integer part of the point $x \in \mathbb{R}^{2}$.

For $x_{i}^{\varepsilon}$ a zero of $v_{\varepsilon}$, let

$$
y_{i}^{\varepsilon}=\frac{\frac{x_{i}^{\varepsilon}}{\delta}-\left[\frac{x_{i}^{\varepsilon}}{\delta}\right]}{\lambda} \in \omega .
$$

Then, as $\varepsilon \rightarrow 0$, up to pass to a subsequence, we have $y_{i}^{\varepsilon} \rightarrow \hat{\hat{a}}_{i} \in \omega$. Here, $\hat{\hat{a}}_{i}$ minimizes a renormalized energy $\tilde{W}_{1}: \omega \rightarrow \mathbb{R}$ (given in [13] Eq. (90)) which depends only on $\omega$ and $b$. In particular, $\hat{\hat{a}}_{i}$ is independent of the boundary condition $g$.

Theorem 5. Assume that $\lambda, \delta$ satisfy (1.3) and if the pinning term is not periodic (represented Figure 2) then we assume also that $\lambda \rightarrow 0$.

We have the following expansion

$$
F_{\varepsilon}\left(v_{\varepsilon}\right)=J_{\varepsilon, \varepsilon}+d b^{2}(\pi \ln b+\gamma)+o_{\varepsilon}(1)
$$

where $J_{\varepsilon, \varepsilon}$ is defined in (3.6) and $\gamma>0$ is the universal constant defined in [4] Lemma IX.1.

This article is divided in two parts: 
- In the first one (Section 3) we consider two auxiliary minimization problems for weighted Dirichlet functionals associated to $\mathbb{S}^{1}$-valued maps.

- The second part (Section 4) is devoted to the proofs of Theorems 1, 2, 3, 4, 5, The main tool is an $\eta$-ellipticity result (Lemma 19). This lemma reduces (under the assumption that $\lambda, \delta$ satisfy (1.3) ) the study of $F_{\varepsilon}$ to the one of the auxiliary problems considered Section 3 ,

\section{Shrinking holes for weighted Dirichlet functionals}

This section is devoted to the study of two minimization problems and it is divided in three subsections.

The first and the second subsections are related with minimizations of weighted Dirichlet functionals among $\mathbb{S}^{1}$-valued maps. In both subsections, the considered weights are the more general one: $\alpha \in L^{\infty}\left(\mathbb{R}^{2},\left[b^{2}, 1\right]\right)$. The third subsection deals the weight $\alpha=U_{\varepsilon}^{2}$ in the situation where $U_{\varepsilon}$ is the minimizer of $E_{\varepsilon}$ in $H_{1}^{1}$ with $a_{\varepsilon}$ represented Figure 1 (the periodic case with or without dilution) or Figure 2 (the general diluted case).

Notation 7. In Section 3 we fix :

- a smooth simply connected domain $\Omega \subset \mathbb{R}^{2}$;

- a boundary condition $g \in C^{\infty}\left(\partial \Omega, \mathbb{S}^{1}\right)$ s.t. $d:=\operatorname{deg}_{\partial \Omega}(g)>0$;

- a smooth and bounded open subset $\Omega^{\prime} \prec \mathbb{R}^{2}$ s.t. $\bar{\Omega} \subset \Omega^{\prime}$;

- an extension of $g$ which is in $C^{\infty}\left(\overline{\Omega^{\prime}} \backslash \Omega, \mathbb{S}^{1}\right)$ (this extension is also denoted by $g$ ).

We will also consider (uniformly bounded) families of points/degres $\left\{\left(x_{1}, d_{1}\right), \ldots,\left(x_{N}, d_{N}\right)\right\}=$ $\{\mathbf{x}, \mathbf{d}\}$ s.t.

- $x_{i} \in \Omega, x_{i} \neq x_{i^{\prime}}$ for $i \neq i^{\prime}$;

- $d_{i}$ are s.t. $d_{i} \in \mathbb{N}^{*}$ and $\sum_{i} d_{i}=d($ thus $N \leq d)$.

According to the considered problems, for $0<\rho \leq 8^{-1} \min _{i \neq i^{\prime}}\left|x_{i}-x_{i^{\prime}}\right|$ we will use the following perforated domains

- $\Omega_{\rho}:=\Omega_{\rho}(\mathbf{x})=\Omega \backslash \cup_{i} \overline{B\left(x_{i}, \rho\right)}$;

- $\Omega_{\rho}^{\prime}:=\Omega_{\rho}^{\prime}(\mathbf{x})=\Omega^{\prime} \backslash \cup_{i} \overline{B\left(x_{i}, \rho\right)}$.

\subsection{Existence results}

In this subsection we prove the existence of solutions of two minimization problems whose studies will be the purpose of the rest of Section 3 (Subsections 3.2 \& 3.3).

\subsubsection{Existence of minimal maps defined in a perforated domain}

Let $\mathbf{x}=\left(x_{1}, \ldots, x_{N}\right)$ be $1 \leq N \leq d$ distinct points of $\Omega$ and let $\mathbf{d}=\left(d_{1}, \ldots, d_{N}\right) \in\left(\mathbb{N}^{*}\right)^{N}$ s.t. $\sum_{i} d_{i}=d$.

For $0<\rho<8^{-1} \min _{i \neq j}\left|x_{i}-x_{j}\right|$, we denote $\Omega_{\rho}=\Omega \backslash \cup \overline{\left(x_{i}, \rho\right)}$. 
We define

$$
\mathcal{I}_{\rho}(\mathbf{x}, \mathbf{d})=\mathcal{I}_{\rho}:=\left\{w \in H^{1}\left(\Omega_{\rho}, \mathbb{S}^{1}\right) \mid w=g \text { on } \partial \Omega \text { and } \operatorname{deg}_{\partial B\left(x_{i}, \rho\right)}(w)=d_{i}\right\}
$$

and for $0<\rho<8^{-1} \min \left\{\min _{i \neq j}\left|x_{i}-x_{j}\right|, \min _{i} \operatorname{dist}\left(x_{i}, \partial \Omega\right)\right\}$

$$
\mathcal{J}_{\rho}(\mathbf{x}, \mathbf{d})=\mathcal{J}_{\rho}:=\left\{w \in H^{1}\left(\Omega_{\rho}, \mathbb{S}^{1}\right) \mid w=g \text { on } \partial \Omega \text { and } w\left(x_{i}+\rho \mathrm{e}^{\imath \theta}\right)=\mathrm{e}^{\imath\left(d_{i} \theta+\theta_{i}\right)}, \theta_{i} \in \mathbb{R}\right\} .
$$

From the compatibility condition $\operatorname{deg}_{\partial \Omega}(g)=d=\sum d_{i}$, we have $\mathcal{I}_{\rho}(\mathbf{x}, \mathbf{d}), \mathcal{J}_{\rho}(\mathbf{x}, \mathbf{d}) \neq \emptyset$ and it is clear that $\mathcal{J}_{\rho}(\mathbf{x}, \mathbf{d}) \subset \mathcal{I}_{\rho}(\mathbf{x}, \mathbf{d})$.

In Subsection 3.2, we compare the minimal energies corresponding to a weighted Dirichlet functional in the above sets. Here, we just state existence results.

Proposition 8. Let $\alpha \in L^{\infty}(\Omega)$ be s.t. $b^{2} \leq \alpha \leq 1$. Consider the minimization problems

$$
\widehat{\mathcal{I}}_{\rho, \alpha}(\mathbf{x}, \mathbf{d})=\inf _{w \in \mathcal{I}_{\rho}} \frac{1}{2} \int_{\Omega_{\rho}} \alpha|\nabla w|^{2}
$$

and

$$
\widehat{\mathcal{J}}_{\rho, \alpha}(\mathbf{x}, \mathbf{d})=\inf _{w \in \mathcal{J}_{\rho}} \frac{1}{2} \int_{\Omega_{\rho}} \alpha|\nabla w|^{2} .
$$

In both minimization problems the infima are attained.

Moreover, if $\alpha \in W^{1, \infty}(\Omega)$, then, denoting $w_{\rho, \alpha}^{\mathrm{deg}}\left(\right.$ resp. $w_{\rho, \alpha}^{\text {Dir }}$ ) a global minimizer of $\frac{1}{2} \int_{\Omega_{\rho}} \alpha|\nabla \cdot|^{2}$ in $\mathcal{I}_{\rho}(\mathbf{x}, \mathbf{d})$ (resp. in $\left.\mathcal{J}_{\rho}(\mathbf{x}, \mathbf{d})\right)$ we have $w_{\rho, \alpha}^{\mathrm{deg}} \in H^{2}\left(\Omega_{\rho}, \mathbb{S}^{1}\right)$ (resp. $w_{\rho, \alpha}^{\text {Dir }} \in$ $\left.H^{2}\left(\Omega_{\rho}, \mathbb{S}^{1}\right)\right)$ and

$$
\begin{gathered}
\left\{\begin{array}{l}
-\operatorname{div}\left(\alpha \nabla w_{\rho, \alpha}^{\mathrm{deg}}\right)=\alpha\left|\nabla w_{\rho, \alpha}^{\mathrm{deg}}\right|^{2} w_{\rho, \alpha}^{\mathrm{deg}} \text { in } \Omega_{\rho} \\
w_{\rho, \alpha}^{\mathrm{deg}} \in \mathcal{I}_{\rho} \text { and } w_{\rho, \alpha}^{\mathrm{deg}} \times \partial_{\nu} w_{\rho, \alpha}^{\mathrm{deg}}=0 \text { on } \partial B\left(x_{i}, \rho\right), i=1, \ldots, N
\end{array},\right. \\
\left\{\begin{array}{l}
-\operatorname{div}\left(\alpha \nabla w_{\rho, \alpha}^{\mathrm{Dir}}\right)=\alpha\left|\nabla w_{\rho, \alpha}^{\text {Dir }}\right|^{2} w_{\rho, \alpha}^{\text {Dir }} \text { in } \Omega_{\rho} \\
w_{\rho, \alpha}^{\text {Dir }} \in \mathcal{J}_{\rho} \text { and } \int_{\partial B\left(x_{i}, \rho\right)} \alpha w_{\rho, \alpha}^{\text {Dir }} \times \partial_{\nu} w_{\rho, \alpha}^{\text {Dir }}=0, i=1, \ldots, N
\end{array}\right.
\end{gathered}
$$

The proof of this standard result is postponed to Appendix A

In the special case $\alpha=U_{\varepsilon}^{2}$, we denote

$$
\widehat{\mathcal{I}}_{\rho, \varepsilon}(\mathbf{x}, \mathbf{d})=\inf _{w \in \mathcal{I}_{\rho}} \frac{1}{2} \int_{\Omega_{\rho}} U_{\varepsilon}^{2}|\nabla w|^{2} \text { and } \widehat{\mathcal{J}}_{\rho, \varepsilon}(\mathbf{x}, \mathbf{d})=\inf _{w \in \mathcal{J}_{\rho}} \frac{1}{2} \int_{\Omega_{\rho}} U_{\varepsilon}^{2}|\nabla w|^{2} .
$$

\subsubsection{Existence of an optimal perforated domain}

For $\alpha \in L^{\infty}\left(\mathbb{R}^{2},\left[b^{2}, 1\right]\right)$ we define

$$
I_{\rho, \alpha}:=\inf _{\substack{x_{1}, \ldots, x_{N} \in \Omega \\\left|x_{i}-x_{j}\right| \geq 8 \rho \\ d_{1}, \ldots, d_{N}>0, \sum d_{i}=d}} \inf _{\substack{w \in H_{g}^{1}\left(\Omega_{\rho}^{\prime}, \mathbb{S}^{1}\right) \\ \operatorname{deg}_{\partial B\left(x_{i}, \rho\right)}(w)=d_{i}}} \frac{1}{2} \int_{\Omega_{\rho}^{\prime}} \alpha|\nabla w|^{2}
$$

and

$$
J_{\rho, \alpha}:=\inf _{\begin{array}{c}
x_{1}, \ldots, x_{d} \in \Omega \\
x_{i}-x_{j} \mid \geq 8 \rho \\
\operatorname{dist}\left(x_{i}, \partial \Omega\right) \geq 8 \rho
\end{array}} \inf _{\substack{w \in H_{g}^{1}\left(\Omega_{\rho}, \mathbb{S}^{1}\right) \\
\left(x_{i}+\rho \mathrm{e}^{2 \theta}\right)=\mathrm{e}^{\left(\theta+\theta_{i}\right)}, \theta_{i} \in \mathbb{R}}} \frac{1}{2} \int_{\Omega_{\rho}} \alpha|\nabla w|^{2} .
$$


Here $\Omega_{\rho}^{\prime}=\Omega^{\prime} \backslash \cup \overline{B\left(x_{i}, \rho\right)}$.

In the special case $\alpha=U_{\varepsilon}^{2}$, we denote

$$
I_{\rho, \varepsilon}:=\inf _{\substack{x_{1}, \ldots, x_{N} \in \Omega \\
\left|x_{i}-x_{j}\right| \geq 8 \rho \\
d_{1}, \ldots, d_{N}>0, \sum d_{i}=d}} \inf _{\begin{array}{c}
w \in H_{g}^{1}\left(\Omega_{\rho}^{\prime}, \mathbb{S}^{1}\right) \\
\operatorname{deg}_{\partial B\left(x_{i}, \rho\right)}(w)=d_{i}
\end{array}} \frac{1}{2} \int_{\Omega_{\rho}^{\prime}} U_{\varepsilon}^{2}|\nabla w|^{2}
$$

and

$$
J_{\rho, \varepsilon}:=\inf _{\begin{array}{c}
x_{1}, \ldots, x_{d} \in \Omega \\
\left|x_{i}-x_{j}\right| \geq 8 \rho \\
\operatorname{dist}\left(x_{i}, \partial \Omega\right) \geq 8 \rho
\end{array}} \inf _{\substack{w \in H_{g}^{1}\left(\Omega_{\rho}, \mathbb{S}^{1}\right) \\
\left(x_{i}+\rho \mathrm{e}^{2 \theta}\right)=\mathrm{e}^{\left(\theta+\theta_{i}\right)}, \theta_{i} \in \mathbb{R}}} \frac{1}{2} \int_{\Omega_{\rho}} U_{\varepsilon}^{2}|\nabla w|^{2} .
$$

We have the following result

Proposition 9. For $\alpha \in L^{\infty}\left(\mathbb{R}^{2},\left[b^{2}, 1\right]\right.$ ), there are $\mathbf{x}_{\rho, \alpha}^{\mathrm{deg}}, \mathbf{x}_{\rho, \alpha}^{\mathrm{Dir}} \in \Omega^{d}$ and $\mathbf{d}_{\rho, \alpha} \in\left(\mathbb{N}^{*}\right)^{N}$ (with $\left.\mathbf{d}_{\rho, \alpha}=\left(d_{1}, \ldots, d_{N}\right), \sum d_{i}=d\right)$ s.t. $\left\{\mathbf{x}_{\rho, \alpha}^{\mathrm{deg}}, \mathbf{d}_{\rho, \alpha}\right\}$ minimizes $I_{\rho, \alpha}$ and $\mathbf{x}_{\rho, \alpha}^{\text {Dir }}$ minimizes $J_{\rho, \alpha}$.

The proof of this result is in Appendix B.

\subsection{Dirichlet Vs Degree Conditions in a fixed perforated domain}

Let $\eta_{\text {stop }}>0$ be s.t. $\eta_{\text {stop }}<10^{-5} \cdot 9^{-d^{2}} \operatorname{diam}(\Omega)$ and let $N \in\{1, \ldots, d\}$.

Consider $x_{1}, \ldots, x_{N} \in \Omega, N$ distinct points of $\Omega$ satisfying the condition $\eta_{\text {stop }}<10^{-3}$. $9^{-d^{2}} \min \operatorname{dist}\left(x_{i}, \partial \Omega\right)$, and let $\rho>0$ be s.t. $\min \left\{\eta_{\text {stop }}, \min _{i \neq j}\left|x_{i}-x_{j}\right|\right\} \geq 8 \rho$. Roughly speaking $\eta_{\text {stop }}$ controls the distance between the points and $\partial \Omega$.

The main result of this section is

Proposition 10. There is $C_{0}>0$ depending only on $g, \Omega, \eta_{\text {stop }}$ and $b$ s.t. for $\alpha \in$ $L^{\infty}\left(\Omega,\left[b^{2}, 1\right]\right)$ we have

$$
\widehat{\mathcal{I}}_{\rho, \alpha}(\mathbf{x}, \mathbf{d}) \leq \widehat{\mathcal{J}}_{\rho, \alpha}(\mathbf{x}, \mathbf{d}) \leq \widehat{\mathcal{I}}_{\rho, \alpha}(\mathbf{x}, \mathbf{d})+C_{0} .
$$

Here, $\widehat{\mathcal{I}}_{\rho, \alpha}$ and $\widehat{\mathcal{J}}_{\rho, \alpha}$ are defined Proposition 8 .

The rigorous proof of Proposition 10 is presented in Appendix C. Here, we simply present the main lines of the proof.

Two situations are possible:

1. $N=1$ or the points $x_{1}, \ldots, x_{N}$ are well separated: $\frac{1}{4} \min _{i \neq j}\left|x_{i}-x_{j}\right|>\eta_{\text {stop }}$,

2. The points $x_{1}, \ldots, x_{N}$ are not well separated: $\frac{1}{4} \min _{i \neq j}\left|x_{i}-x_{j}\right| \leq \eta_{\text {stop }}$.

If the points are well separated (or $N=1$ ), Proposition 10 can be easily proved: it is a direct consequence of Proposition 45 and Lemma 44 in Appendix C. These results, whose statements and proofs are postponed in Appendix C, give essentially the existence of test functions into two kinds of domains.

The domains are

- the thin domain $\Omega_{10^{-1}} \eta_{\text {stop }}(\mathbf{x})=\Omega \backslash \cup \overline{B\left(x_{i}, 10^{-1} \eta_{\text {stop }}\right)}$ obtained by perforating $\Omega$ by "large", "well separated" and "far from $\partial \Omega$ " discs,

- the thick annulars $B\left(x_{i}, 10^{-1} \eta_{\text {stop }}\right) \backslash \overline{B\left(x_{i}, \rho\right)}$. 
The proof is made in three steps:

Step 1: Using Lemma 44, we obtain a constant $C_{1}$ (depending only on $g, \Omega, \eta_{\text {stop }}$ ) s.t.

$$
\widehat{\mathcal{J}}_{10^{-1} \eta_{\text {stop }, \alpha}}(\mathbf{x}, \mathbf{d}) \leq C_{1} \text {. }
$$

Step 2: With the help of Proposition 45, we obtain the existence of a constant $C_{b}$ (depending only on $b)$ s.t. for $\tilde{d} \in \mathbb{N}$, denoting $A_{\rho}^{i}=B\left(x_{i}, 10^{-1} \eta_{\text {stop }}\right) \backslash \overline{B\left(x_{i}, \rho\right)}$, we have

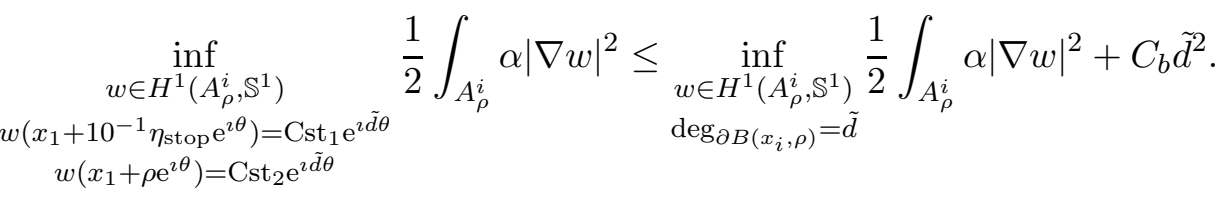

Step 3: By extending a minimizer of $\widehat{\mathcal{J}}_{10^{-1} \eta_{\text {stop }}, \alpha}(\mathbf{x}, \mathbf{d})$ by the ones of $\frac{1}{2} \int_{A_{\rho}^{i}} \alpha|\nabla \cdot|^{2}$ with Dirichlet conditions, we can construct a map which proves the result taking $C_{0}=$ $C_{1}+d^{3} C_{b}$.

\subsection{Optimal perforated domains for the degree conditions}

Recall that we fixed $\Omega^{\prime} \supset \Omega$ a smooth bounded domain s.t. $\operatorname{dist}\left(\partial \Omega^{\prime}, \Omega\right)>0$ and a smooth $\mathbb{S}^{1}$-valued extension of $g$ to $\Omega^{\prime} \backslash \bar{\Omega}$ (still denoted by $g$ ).

In this section, we study the minimization problem

$$
I_{\rho, \varepsilon}:=\inf _{\substack{x_{1}, \ldots, x_{N} \in \Omega \\\left|x_{i}-x_{j}\right| \geq 8 \rho \\ d_{1}, \ldots, d_{N}>0, \sum d_{i}=d}} \inf _{\substack{w \in H_{g}^{1}\left(\Omega_{\rho}^{\prime}, \mathbb{S}^{1}\right) \\ \operatorname{deg}_{\partial B\left(x_{i}, \rho\right)}(w)=d_{i}}} \frac{1}{2} \int_{\Omega_{\rho}^{\prime}} U_{\varepsilon}^{2}|\nabla w|^{2}
$$

where

$$
\Omega_{\rho}^{\prime}=\Omega^{\prime} \backslash \cup \overline{B\left(x_{i}, \rho\right)}
$$

and

$$
H_{g}^{1}\left(\Omega_{\rho}^{\prime}, \mathbb{S}^{1}\right)=\left\{w \in H^{1}\left(\Omega_{\rho}^{\prime}, \mathbb{S}^{1}\right) \mid w=g \text { in } \Omega^{\prime} \backslash \overline{\Omega \cup B\left(x_{i}, \rho\right)}\right\} ;
$$

here, we extended $U_{\varepsilon}$ with the value 1 outside $\Omega$. We recall that we denoted by $U_{\varepsilon}$ the unique global minimizer of $E_{\varepsilon}$ in $H_{1}^{1}$.

In this subsection we assume that Hypothesis (1.3) holds $\left(|\ln (\lambda \delta)|^{3} /|\ln \varepsilon| \rightarrow 0\right)$. This is not optimal for the statements but it makes the proofs simpler (this hypothesis may be relaxed, but it appears as a crucial and technical hypothesis for the methods developed Section 4).

A first purpose of this section is the study of the behavior of $I_{\rho, \varepsilon}$ when $\rho=\rho(\varepsilon) \rightarrow 0$ as $\varepsilon \rightarrow 0$. In view of the application we have in mind we suppose that $\lambda \delta^{P+1} \gg \rho(\varepsilon) \geq \varepsilon$ but this is not crucial for our arguments (here $P=1$ if $U_{\varepsilon}$ is associated associated with the periodic pinning term) .

A second objective of our study is to exhibit the behavior of almost minimal configurations $\left\{\left(x_{1}^{n}, \ldots, x_{N}^{n}\right),\left(d_{1}^{n}, \ldots, d_{N}^{n}\right)\right\}$.

For fixed $\rho, \varepsilon$, the existence of a minimal configuration of points $\mathbf{x}_{\rho, \varepsilon}$ is the purpose of Proposition 9, In this section we consider only almost minimal configurations. 
Notation 11. For $\varepsilon_{n} \downarrow 0$, we say that $\left\{\left(x_{1}^{n}, \ldots, x_{N}^{n}\right),\left(d_{1}^{n}, \ldots, d_{N}^{n}\right)\right\}$ is an almost minimal configuration for $\rho=\rho\left(\varepsilon_{n}\right) \downarrow 0$ when $x_{1}^{n}, \ldots, x_{N}^{n} \in \Omega,\left|x_{i}^{n}-x_{j}^{n}\right| \geq 8 \rho, d_{1}^{n}, \ldots, d_{N}^{n}>0, \sum d_{i}^{n}=$ $d$ and there is $C>0$ (independent of $n$ ) s.t.

$$
\inf _{\substack{w \in H_{g}^{1}\left(\Omega_{\rho}^{\prime}, \mathbb{S}^{1}\right) \\ \operatorname{deg}_{\partial B\left(x_{i}^{n}, \rho\right)}(w)=d_{i}^{n}}} \frac{1}{2} \int_{\Omega_{\rho}^{\prime}} U_{\varepsilon_{n}}^{2}|\nabla w|^{2}-I_{\rho, \varepsilon_{n}} \leq C .
$$

Roughly speaking, we establish in this section two repelling effects for the points: point/point and point $/ \partial \Omega$; and an attractive effect for the points by the inclusions $\omega_{\varepsilon}$.

\subsubsection{The case of the periodic pinning term}

The main result of this section establishes that when $\varepsilon_{n}, \rho \downarrow 0$, an almost minimal configuration $\left\{\left(x_{1}^{n}, \ldots, x_{N}^{n}\right),\left(d_{1}^{n}, \ldots, d_{N}^{n}\right)\right\}$ is s.t. (for sufficiently large $n$ )

- the points $x_{i}^{n}$ 's cannot be mutually close,

- the degrees $d_{i}^{n}$ 's are necessarily all equal to 1 ,

- the points $x_{i}^{n}$ 's cannot approach $\partial \Omega$,

- there is $c>0$ s.t. $B\left(x_{i}^{n}, c \lambda \delta\right) \subset \omega_{\varepsilon}$ for all $i$.

These facts are expressed in the following proposition (whose proof is postponed to Appendix D).

Proposition 12. [The case of a periodic pinning term]

Assume that $\lambda, \delta$ satisfy (1.3) and let $a_{\varepsilon}$ be the periodic the pinning term (represented Figure 11).

Let $\varepsilon_{n} \downarrow 0, \rho=\rho\left(\varepsilon_{n}\right) \downarrow 0, x_{1}^{n}, \ldots, x_{N}^{n} \in \Omega$ be s.t. $\left|x_{i}^{n}-x_{j}^{n}\right| \geq 8 \rho, \rho \geq \varepsilon_{n}$ and let $d_{1}^{n}, \ldots, d_{N}^{n} \in \mathbb{N}^{*}$ be s.t. $\sum d_{i}^{n}=d$.

1. Assume that there is $i_{0} \in\{1, \ldots, N\}$ s.t. $d_{i_{0}}^{n} \neq 1$ or that there are $i_{0} \neq j_{0}$ s.t. $\left|x_{i_{0}}^{n}-x_{j_{0}}^{n}\right| \rightarrow 0$. Then

$$
\inf _{\substack{w \in H_{g}^{1}\left(\Omega_{\rho}^{\prime}, \mathbb{S}^{1}\right) \\ \operatorname{deg}_{\partial B\left(x_{i}^{n}, \rho\right)}(w)=d_{i}^{n}}}\left\{\frac{1}{2} \int_{\Omega_{\rho}^{\prime}} U_{\varepsilon_{n}}^{2}|\nabla w|^{2}-I_{\rho, \varepsilon_{n}}\right\} \rightarrow \infty .
$$

2. Assume that there is $i_{0} \in\{1, \ldots, N\}$ s.t. $\operatorname{dist}\left(x_{i_{0}}^{n}, \partial \Omega\right) \rightarrow 0$. Then

$$
\inf _{\substack{w \in H_{g}^{1}\left(\Omega_{\rho}^{\prime}, \mathbb{S}^{1}\right) \\ \operatorname{deg}_{\partial B\left(x_{i}^{n}, \rho\right)}(w)=d_{i}^{n}}}\left\{\frac{1}{2} \int_{\Omega_{\rho}^{\prime}} U_{\varepsilon_{n}}^{2}|\nabla w|^{2}-I_{\rho, \varepsilon_{n}}\right\} \rightarrow \infty .
$$

3. Assume that $\frac{\rho}{\lambda \delta} \rightarrow 0$ and that there is $i_{0} \in\{1, \ldots, N\}$ s.t. $x_{i_{0}}^{n} \notin \omega_{\varepsilon}$ or s.t. $x_{i_{0}}^{n} \in \omega_{\varepsilon}$ and $\frac{\operatorname{dist}\left(x_{i_{0}}^{n}, \partial \omega_{\varepsilon}\right)}{\lambda \delta} \rightarrow 0$. Then

$$
\inf _{\substack{w \in H_{g}^{1}\left(\Omega_{\rho}^{\prime}, \mathbb{S}^{1}\right) \\ \operatorname{deg}_{\partial B\left(x_{i}^{n}, \rho\right)}(w)=d_{i}^{n}}}\left\{\frac{1}{2} \int_{\Omega_{\rho}^{\prime}} U_{\varepsilon_{n}}^{2}|\nabla w|^{2}-I_{\rho, \varepsilon_{n}}\right\} \rightarrow \infty .
$$


A straightforward consequence of Proposition 12 is the following

Corollary 13. 1. Consider an almost minimal configuration $\left\{\mathbf{x}_{\rho, \varepsilon}, \mathbf{d}_{\rho, \varepsilon}\right\} \in \Omega^{N} \times \mathbb{N}^{* N}$, i.e., assume that there is $w_{\rho, \varepsilon} \in H_{g}^{1}\left(\Omega^{\prime} \backslash \cup \overline{B\left(x_{i}^{\rho, \varepsilon}, \rho\right)}, \mathbb{S}^{1}\right)$ verifying

$$
\operatorname{deg}_{\partial B\left(x_{i}^{\rho, \varepsilon}, \rho\right)}(w)=d_{i}^{\rho, \varepsilon} \text { and } \frac{1}{2} \int_{\Omega^{\prime} \backslash \cup \frac{}{B\left(x_{i}^{\rho, \varepsilon}, \rho\right)}} U_{\varepsilon}^{2}|\nabla w|^{2} \leq I_{\rho, \varepsilon}+C .
$$

(Here, $C$ is independent of $\varepsilon$.)

Then, there is some $\eta_{0}$ independent of $\varepsilon$ s.t., for small $\varepsilon$, we have

$$
\left|x_{i}^{\rho, \varepsilon}-x_{j}^{\rho, \varepsilon}\right|, \operatorname{dist}\left(x_{i}^{\rho, \varepsilon}, \partial \Omega\right) \geq \eta_{0} \text { and } d_{i}=1 \text { for all } i \neq j, i, j \in\{1, \ldots, N\} .
$$

In particular, we have $N=d$.

2. If, in addition, $\rho=\rho(\varepsilon)$ is s.t. $\rho \geq \varepsilon$ and $\frac{\rho}{\lambda \delta} \rightarrow 0$, then there is $c>0$ (independent of $\varepsilon$ ) s.t., for small $\varepsilon$, we have $B\left(x_{i}^{\rho, \varepsilon}, c \lambda \delta\right) \subset \omega_{\varepsilon}$.

Proof of Corollary [13. We prove the first part. Let $C>0$. We argue by contradiction and we assume that for all $n \in \mathbb{N}^{*}$ there are $0<\varepsilon_{n} \leq \rho=\rho\left(\varepsilon_{n}\right) \leq 1 / n, \mathbf{x}_{n}=\mathbf{x}_{\rho, \varepsilon_{n}},\left(d_{1}, \ldots, d_{N}\right)$ and $w_{n}=w_{\rho, \varepsilon_{n}}$ satisfying the hypotheses of Corollary 13 and s.t.

$\min \left\{\left|x_{i}^{n}-x_{j}^{n}\right|, \operatorname{dist}\left(x_{i}^{n}, \partial \Omega\right)\right\} \rightarrow 0$ or s.t. there is $i \in\{1, \ldots, N\}$ for which we have $d_{i} \neq 1$.

By construction we have that $\left\{\mathbf{x}_{\rho, \varepsilon_{n}}, \mathbf{d}\right\}$ is an almost minimal configuration for $I_{\rho, \varepsilon_{n}}$ with $\rho=\rho\left(\varepsilon_{n}\right) \geq \varepsilon_{n}$. Clearly from Proposition 12 we find a contradiction.

The proof of the second part is similar.

We end this subsection by the following direct consequence of Corollary 13

Corollary 14. For sufficiently small $\varepsilon, \rho$, an almost minimal configuration $\left(x_{1}, \ldots, x_{d}\right)$ for $J_{\rho, \varepsilon}$ is an almost minimal configuration for $I_{\rho, \varepsilon}$.

Moreover, there is $C_{0}>0$ s.t. $J_{\rho, \varepsilon} \leq I_{\rho, \varepsilon}+C_{0}, C_{0}$ is independent of small $\varepsilon, \rho$.

Proof. Let $C \geq 0$ and let $\left(x_{1}, \ldots, x_{d}\right),\left(x_{1}^{\prime}, \ldots, x_{d}^{\prime}\right) \in \Omega^{d}$ be s.t.

$$
\hat{\mathcal{J}}_{\rho, \varepsilon}\left(x_{1}, \ldots, x_{d}\right) \leq J_{\rho, \varepsilon}+C
$$

and

$$
\hat{\mathcal{I}}_{\rho, \varepsilon}\left(x_{1}^{\prime}, \ldots, x_{d}^{\prime}\right) \leq I_{\rho, \varepsilon}+C .
$$

From Corollary 13, there is $\eta_{0}=\eta_{0}(C)>0$ s.t. for $\varepsilon \leq \rho \leq \eta_{0}, \min _{i} \operatorname{dist}\left(x_{i}^{\prime}, \partial \Omega\right) \geq \eta_{0}$. Using Proposition 10 we have the existence of $C_{0}$ s.t.

$$
\begin{aligned}
\hat{\mathcal{I}}_{\rho, \varepsilon}\left(x_{1}, \ldots, x_{d}\right) \leq \hat{\mathcal{J}}_{\rho, \varepsilon}\left(x_{1}, \ldots, x_{d}\right) & \leq J_{\rho, \varepsilon}+C \leq \hat{\mathcal{J}}_{\rho, \varepsilon}\left(x_{1}^{\prime}, \ldots, x_{d}^{\prime}\right)+C \\
& \leq \hat{\mathcal{I}}_{\rho, \varepsilon}\left(x_{1}^{\prime}, \ldots, x_{d}^{\prime}\right)+C+C_{0} \\
& \leq I_{\rho, \varepsilon}+2 C+C_{0} .
\end{aligned}
$$




\subsubsection{A more precise result for the case of the periodic pinning term with dilution}

In this section we focus on the periodic pinning term (represented Figure 1) with dilution: $\lambda \rightarrow 0$.

Notation 15. We define two kinds of configuration of distinct points of $\Omega$ :

- We say that for $\varepsilon_{n} \downarrow 0$ and $\rho=\rho\left(\varepsilon_{n}\right) \rightarrow 0, d$ distinct points of $\Omega, \mathbf{x}_{n}=\left(x_{1}^{n}, \ldots, x_{d}^{n}\right)$ form a quasi-minimizer of $J_{\rho, \varepsilon_{n}}$ when $\mathcal{J}_{\rho, \varepsilon_{n}}\left(\mathbf{x}_{n}\right)-J_{\rho, \varepsilon_{n}} \rightarrow 0$.

- We say that for $\varepsilon_{n} \downarrow 0$ and $\rho=\rho\left(\varepsilon_{n}\right) \rightarrow 0, d$ distinct points of $\Omega, \mathbf{x}_{n}=\left(x_{1}^{n}, \ldots, x_{d}^{n}\right)$ form a quasi-minimizer of $W_{g}$, the renormalized energy of Bethuel-Brezis-Hélein (see [4]) when $W_{g}\left(\mathbf{x}_{n}\right) \rightarrow \min W_{g}$.

Proposition 16. [Asymptotic location of optimal perforations]

Assume that $\lambda, \delta$ satisfy (1.3) and that $\lambda \rightarrow 0$.

Let $\varepsilon_{n} \downarrow 0, \rho=\rho\left(\varepsilon_{n}\right) \rightarrow 0, \rho \geq \varepsilon_{n}$ and $\mathbf{x}_{n}=\left(x_{1}^{n}, \ldots, x_{d}^{n}\right)$ be d distinct points of $\Omega$.

If the points $\mathbf{x}_{n}$ form a quasi-minimizer of $J_{\rho, \varepsilon_{n}}$, then $\mathbf{x}_{n}=\left(x_{1}^{n}, \ldots, x_{d}^{n}\right)$ form a quasiminimizer of $W_{g}$.

This proposition is proved Appendix E

\subsubsection{The case of a general pinning term with variable sizes of inclusions}

We assume that $a_{\varepsilon}$ is the general pinning term represented Figure 2 with the hypothesis on the dilution: $\lambda \rightarrow 0$.

Proposition 17. [The case of a non-periodic pinning term]

Assume that $\lambda, \delta$ satisfy (1.3) and $\lambda \rightarrow 0$.

Let $\rho=\rho(\varepsilon)$ s.t. $\rho \geq \varepsilon$ and $\frac{\rho}{\lambda \delta^{3 / 2}} \rightarrow 0$. If $\left\{\mathbf{x}_{\rho, \varepsilon}, \mathbf{d}_{\rho, \varepsilon}\right\}$ is an almost minimal configuration for $I_{\rho, \varepsilon}$, then $N=d$ (thus $d_{i}=1$ for all $i$ ) and there are $c, \eta_{0}>0$ (independent of $\varepsilon$ ) s.t. for sufficiently small $\varepsilon$ :

1. $\left|x_{i}^{\rho, \varepsilon}-x_{j}^{\rho, \varepsilon}\right|, \operatorname{dist}\left(x_{i}^{\rho, \varepsilon}, \partial \Omega\right) \geq \eta_{0}$ for all $i \neq j, i, j \in\{1, \ldots, N\}$.

2. $B\left(x_{i}^{\rho, \varepsilon}, c \lambda \delta\right) \subset \omega_{\varepsilon}$ (the centers of the holes are included in the largest inclusions).

Moreover, there is $C_{0}>0$ s.t. $J_{\rho, \varepsilon} \leq I_{\rho, \varepsilon}+C_{0}, C_{0}$ is independent of small $\varepsilon, \rho$. And thus an almost minimal configuration $\mathbf{x}_{\rho, \varepsilon}$ for $J_{\rho, \varepsilon}$ is an almost minimal configuration for $I_{\rho, \varepsilon}$

This proposition is proved Appendix E(Subsection E.3).

\section{The pinned Ginzburg-Landau functional}

In this section, we turn to the main purpose of this article: the study of minimizers of $E_{\varepsilon}$ (defined in (1.1) ) in $H_{g}^{1}$.

The pinning term is the periodic one (represented Figure 1) or the non periodic one (represented Figure 21).

Recall that we fix $\delta=\delta(\varepsilon), \delta \rightarrow 0, \lambda=\lambda(\varepsilon), \lambda \equiv 1$ or $\lambda \rightarrow 0$ satisfying (1.3). If the pinning term is not periodic then we add the hypothesis $\lambda \rightarrow 0$. 


\subsection{Sharp Upper Bound, $\eta$-ellipticity and Uniform Convergence}

\subsubsection{Sharp Upper Bound and an $\eta$-ellipticity result}

We may easily prove the following upper bound.

Lemma 18. Assume that $\frac{\rho}{\lambda \delta} \rightarrow 0$ (or $\frac{\rho}{\lambda \delta^{3 / 2}} \rightarrow 0$ if the pinning term is not periodic), then we have

$$
\inf _{v \in H_{g}^{1}(\Omega, \mathbb{C})} F_{\varepsilon}(v) \leq d b^{2}\left(\pi \ln \frac{b \rho}{\varepsilon}+\gamma\right)+J_{\rho, \varepsilon}+o_{\varepsilon}(1),
$$

where $\gamma>0$ is a universal constant defined in [4], Lemma IX.1.

Proof. We construct a suitable test function $\tilde{w}_{\varepsilon} \in H_{g}^{1}$ (for sufficiently small $\varepsilon$ ).

From Proposition 9, one may consider $\left(x_{1}^{\varepsilon}, \ldots, x_{d}^{\varepsilon}\right)=\mathbf{x}^{\varepsilon} \in \Omega^{d}$, a minimal configuration for $J_{\rho, \varepsilon}$.

Note that since $\frac{\rho}{\lambda \delta} \rightarrow 0$ (or $\frac{\rho}{\lambda \delta^{3 / 2}} \rightarrow 0$ if the pinning term is not periodic), from Corollaries $13 \& 14$ (or Proposition 17 if the pinning term is not periodic), there are $\eta>0$ and $c>0$ s.t. for small $\varepsilon$ we have $B\left(x_{i}^{\varepsilon}, c \lambda \delta\right) \subset \omega_{\varepsilon}$ and $\min _{i}\left\{\min _{i \neq j}\left|x_{i}-x_{j}\right|, \operatorname{dist}\left(x_{i}, \partial \Omega\right)\right\} \geq \eta$.

Let $w_{\varepsilon}$ be a minimal map in $\mathcal{J}_{\rho, \varepsilon}\left(\mathbf{x}^{\varepsilon}, \mathbf{1}\right)$ (Proposition 8 ). We denote $\mathbf{1}:=(1, \ldots, 1) \in \mathbb{N}^{d}$

Let $u_{\varepsilon /(b \rho)} \in H^{1}(B(0,1), \mathbb{C})$ be a global minimizer of

$$
E_{\varepsilon /(b \rho)}^{0}(u)=\frac{1}{2} \int_{B(0,1)}\left\{|\nabla u|^{2}+\frac{b^{2} \rho^{2}}{2 \varepsilon^{2}}\left(1-|u|^{2}\right)^{2}\right\}, u \in H_{x /|x|}^{1}(B(0,1), \mathbb{C}) .
$$

We consider the test function

$$
\tilde{w}_{\varepsilon}(x)=\left\{\begin{array}{ll}
w_{\varepsilon} & \text { in } \Omega_{\rho} \\
\alpha_{i}^{\varepsilon} u_{\varepsilon /(b \rho)}\left(\frac{x-x_{i}^{\varepsilon}}{\rho}\right) & \text { in } B\left(x_{i}^{\varepsilon}, \rho\right)
\end{array} .\right.
$$

Here the constants $\alpha_{i}^{\varepsilon} \in \mathbb{S}^{1}$ are s.t. $w_{\varepsilon}\left(x_{i}^{\varepsilon}+\rho \mathrm{e}^{\imath \theta}\right)=\alpha_{i}^{\varepsilon} \mathrm{e}^{\imath \theta}$.

Estimate (4.1) is obtained by using the fact that $E_{\varepsilon}^{0}\left(u_{\varepsilon}\right)=\pi|\ln \varepsilon|+\gamma+o_{\varepsilon}(1)$ as $\varepsilon \rightarrow 0$ (see 4] Lemma IX.1) and Proposition 3.

Note that

$$
I_{\rho, \varepsilon} \leq J_{\rho, \varepsilon} \leq \pi d|\ln \rho|+C
$$

We now turn to the $\eta$-ellipticity.

We denote by $v_{\varepsilon}$ a global minimizer of $F_{\varepsilon}$ in $H_{g}^{1}$. We extend $\left|v_{\varepsilon}\right|$ with the value 1 outside $\Omega$.

One of the main ingredients in this work is the following result.

Lemma 19. [ $\eta$-ellipticity lemma]

Let $0<\alpha<1 / 2$. Then the following results hold:

1. If for $\varepsilon<\varepsilon_{0}$

$$
F_{\varepsilon}\left(v_{\varepsilon}, B\left(x, \varepsilon^{\alpha}\right) \cap \Omega\right) \leq \chi^{2}|\ln \varepsilon|-C_{1},
$$

then we have

$$
\left|v_{\varepsilon}\right| \geq 1-C \chi \text { in } B\left(x, \varepsilon^{2 \alpha}\right) .
$$

Here, $\chi_{\varepsilon} \in(0,1)$ is s.t. $\chi_{\varepsilon} \rightarrow 0$ and $\varepsilon_{0}>0, C>0, C_{1}>0$ depend only on $b, \alpha, \chi, \Omega,\|g\|_{C^{1}(\partial \Omega)}$. 
2. If for $\varepsilon<\varepsilon_{0}$

$$
F_{\varepsilon}\left(v_{\varepsilon}, B\left(x, \varepsilon^{\alpha}\right) \cap \Omega\right) \leq C|\ln \varepsilon|
$$

then we have

$$
\left|v_{\varepsilon}\right| \geq \mu \text { in } B\left(x, \varepsilon^{2 \alpha}\right) .
$$

Here, $\mu \in(0,1)$ and $\varepsilon_{0}, C>0$ depend only on $b, \alpha, \mu, \Omega,\|g\|_{C^{1}(\partial \Omega)}$.

This result is a direct consequence of Lemma 1 in [13].

\subsubsection{Uniform convergence of $\left|v_{\varepsilon}\right|$ outside $\omega_{\varepsilon}$}

With the help of Lemma 19, we are in position to establish uniform convergence of $\left|v_{\varepsilon}\right|$ to 1 far away from $\overline{\omega_{\varepsilon}}$.

Proposition 20. Let $10^{-2} \cdot \operatorname{dist}(\omega, \partial Y)>\mu>0$ and $K_{\varepsilon}^{\mu}=\left\{x \in \Omega \mid \operatorname{dist}\left(x, \omega_{\varepsilon}\right) \geq \mu \lambda \delta\right\}$. Then, for sufficiently small $\varepsilon$, we have

$$
\left|v_{\varepsilon}\right| \geq 1-C \sqrt{\frac{|\ln (\lambda \delta)|}{|\ln \varepsilon|}} \text { in } K_{\varepsilon}^{\mu} .
$$

Here $C$ is independent of $\varepsilon$ and $\mu$.

Furthermore, if for some small $\varepsilon$, we have $\left|v_{\varepsilon}(x)\right|<1-C \sqrt{\frac{|\ln (\lambda \delta)|}{|\ln \varepsilon|}}$, then

$$
F_{\varepsilon}\left(v_{\varepsilon}, B\left(x, \varepsilon^{1 / 4}\right)\right) \geq \frac{2(\pi d+1)}{b^{2}\left(1-b^{2}\right)}|\ln (\lambda \delta)|
$$

Proof. Using Lemma [19, 1 with $\alpha=1 / 4$ and $\chi=\sqrt{\frac{2(\pi d+1)}{b^{2}\left(1-b^{2}\right)} \frac{|\ln (\lambda \delta)|}{|\ln \varepsilon|}}$, we obtain the existence of $C>0$ s.t. for $\varepsilon>0$ sufficiently small:

$$
\text { if } F_{\varepsilon}\left(v_{\varepsilon}, B\left(x, \varepsilon^{1 / 4}\right)\right)<\frac{2(\pi d+1)}{b^{2}\left(1-b^{2}\right)}|\ln (\lambda \delta)| \text {, then we have }\left|v_{\varepsilon}\right| \geq 1-C \chi \text { in } B\left(x, \varepsilon^{1 / 2}\right) \text {. }
$$

In order to prove Proposition 20, we argue by contradiction. There are $\varepsilon_{n} \downarrow 0, \mu>0$ and $x_{n} \in K_{\varepsilon_{n}}^{\mu}$ s.t.

$$
\left|v_{\varepsilon_{n}}\left(x_{n}\right)\right|<1-C \chi
$$

From (1.5), we find

$$
\left|U_{\varepsilon_{n}}-1\right| \leq C_{0} \mathrm{e}^{-\frac{\alpha \mu}{2 \xi}} \text { in } K_{\varepsilon_{n}}^{\mu / 2}, \xi=\frac{\varepsilon_{n}}{\lambda \delta} .
$$

Consequently, Lemma 19, the definition of $C$ and (4.3) imply that for large $n$,

$$
\frac{1}{2} \int_{B\left(x_{n}, \varepsilon_{n}^{1 / 4}\right)}\left\{\left|\nabla v_{\varepsilon_{n}}\right|^{2}+\frac{1}{2 \varepsilon_{n}^{2}}\left(1-\left|v_{\varepsilon_{n}}\right|^{2}\right)^{2}\right\} \geq \frac{2(\pi d+1)}{b^{2}\left(1-b^{2}\right)}|\ln (\lambda \delta)|+o_{\varepsilon}(1) .
$$

We extend $v_{\varepsilon}$ to $\Omega^{\prime}:=\Omega+B(0,1)$ with the help of a fixed smooth $\mathbb{S}^{1}$-valued map $v$ s.t. $v=g$ on $\partial \Omega$. We also extend $U_{\varepsilon}$ and $a_{\varepsilon}$ with the value 1 outside $\Omega$.

For $n$ sufficiently large, we have

$$
\frac{1}{2} \int_{\Omega^{\prime}}\left\{\left|\nabla v_{\varepsilon_{n}}\right|^{2}+\frac{1}{2 \varepsilon_{n}^{2}}\left(1-\left|v_{\varepsilon_{n}}\right|^{2}\right)^{2}\right\} \leq C\left|\ln \varepsilon_{n}\right|
$$


Theorem 4.1 in [20] applied with $r=10^{-2} \cdot \lambda \delta \mu$ and for large $n$, implies the existence of $\mathcal{B}^{n}=\left\{B_{j}^{n}\right\}$ a finite disjoint covering by balls of

$$
\left\{x \in \Omega^{\prime} \mid \operatorname{dist}\left(x, \partial \Omega^{\prime}\right)>\frac{\varepsilon_{n}}{b} \text { and } 1-\left|v_{\varepsilon_{n}}(x)\right| \geq\left(\frac{\varepsilon_{n}}{b}\right)^{1 / 8}\right\}
$$

s.t.

$$
\operatorname{rad}\left(\mathcal{B}^{n}\right) \leq 10^{-2} \cdot \lambda \delta \mu
$$

satisfying

$$
\begin{aligned}
\frac{1}{2} \int_{\cup B_{j}^{n}}\left\{\left|\nabla v_{\varepsilon_{n}}\right|^{2}+\frac{b^{2}}{2 \varepsilon_{n}^{2}}\left(1-\left|v_{\varepsilon_{n}}\right|^{2}\right)^{2}\right\} & \geq \pi \sum_{j} d_{j}^{n}\left(\left|\ln \varepsilon_{n}\right|-|\ln (\lambda \delta)|\right)-C \\
& =\pi \sum_{j} d_{j}^{n}|\ln \xi|-C .
\end{aligned}
$$

Here, $\operatorname{rad}\left(\mathcal{B}^{n}\right)=\sum_{j} \operatorname{rad}\left(B_{j}^{n}\right), \operatorname{rad}(B)$ stands for the radius of the ball $B, \xi=\varepsilon_{n} /(\lambda \delta)$ and the integers $d_{j}^{n}$ are defined by

$$
d_{j}^{n}= \begin{cases}\left|\operatorname{deg}_{\partial B_{j}^{n}}\left(v_{\varepsilon_{n}}\right)\right| & \text { if } B_{j}^{n} \subset\left\{x \in \Omega^{\prime} \mid \operatorname{dist}\left(x, \partial \Omega^{\prime}\right)>\frac{\varepsilon_{n}}{b}\right\} . \\ 0 & \text { otherwise }\end{cases}
$$

Since $B_{j} \subset \Omega+B_{1 / 2} \subset\left\{x \in \Omega^{\prime} \mid \operatorname{dist}\left(x, \partial \Omega^{\prime}\right)>\frac{\varepsilon_{n}}{b}\right\}$, we obtain

$$
\frac{1}{2} \int_{\cup B_{j}^{n}}\left\{\left|\nabla v_{\varepsilon_{n}}\right|^{2}+\frac{b^{2}}{2 \varepsilon_{n}^{2}}\left(1-\left|v_{\varepsilon_{n}}\right|^{2}\right)^{2}\right\} \geq \pi d|\ln \xi|-C .
$$

From (4.3) and (1.3) we have

$$
\begin{aligned}
F_{\xi}\left(v_{\varepsilon_{n}}, \cup_{j} B_{j} \cup B\left(x_{n}, \varepsilon_{n}^{1 / 4}\right)\right) \geq & \frac{b^{2}\left(1-b^{2}\right)}{2} \int_{B\left(x_{n}, \varepsilon_{n}^{1 / 4}\right)}\left\{\left|\nabla v_{\varepsilon_{n}}\right|^{2}+\frac{1}{2 \varepsilon_{n}^{2}}\left(1-\left|v_{\varepsilon_{n}}\right|^{2}\right)^{2}\right\}+ \\
& +\frac{b^{2}}{2} \int_{\cup_{j} B_{j}}\left\{\left|\nabla v_{\varepsilon_{n}}\right|^{2}+\frac{b^{2}}{2 \varepsilon_{n}^{2}}\left(1-\left|v_{\varepsilon_{n}}\right|^{2}\right)^{2}\right\}+o_{n}(1) .
\end{aligned}
$$

By combining (4.1) (with $\rho=\lambda^{2} \delta^{2}$ ), (4.2), (4.4), (4.5) and (4.6), we find that

$$
\begin{aligned}
\pi d b^{2} \ln [(\lambda \delta) / \xi]+\pi d\left|\ln \left[(\lambda \delta)^{2}\right]\right| & \geq F_{\varepsilon_{n}}\left(v_{\varepsilon_{n}}, \Omega^{\prime}\right)-\mathcal{O}_{n}(1) \\
& \geq F_{\varepsilon_{n}}\left(v_{\varepsilon_{n}}, \cup_{j} B_{j} \cup B\left(x_{n}, \varepsilon_{n}^{1 / 4}\right)\right)-\mathcal{O}_{n}(1) \\
& \geq \pi d b^{2}|\ln \xi|+2(\pi d+1)|\ln (\lambda \delta)|-\mathcal{O}_{n}(1)
\end{aligned}
$$

which is a contradiction. This completes the proof of Proposition 20 .

\subsection{Bad discs}

\subsubsection{Construction and first properties of bad discs}

A fundamental tool in this article is the use of ad-hoc coverings of $\left\{\left|v_{\varepsilon}\right| \leq 7 / 8\right\}$ by small discs. The best radius for a covering of $\left\{\left|v_{\varepsilon}\right| \leq 7 / 8\right\}$ should be of the order $\varepsilon$. But the construction of such covering need some preliminary results. 
Roughly speaking, the way to get a "sharp" covering is to consider a trivial covering and to "clean" it by dropping some discs with the help an "energetic test" ( $\eta$-ellipticity result).

Here, we used two kinds of energetic tests: Lemma 19 and Theorem III.3 in [4]. Theorem III.3 in [4] gives the most precise results (it allows to deal with discs with radius $\mathcal{O}(\varepsilon)$ ) but it needs a bound on the potential part $\varepsilon^{-2} \int_{\Omega}\left(1-\left|v_{\varepsilon}\right|^{2}\right)^{2}$ which is the purpose of Proposition 30. In order to prove this bound (Proposition 30), we first use larger discs (discs with radius $\rho, \varepsilon \ll \rho \ll \lambda \delta^{P+1}$ ). The construction of intermediate coverings is done via Lemma 19.

We first consider

Notation 21. A trivial covering of $\Omega$ by discs

For $\varepsilon>0$, we fix a family of $\operatorname{discs}\left(B\left(x_{i}, \varepsilon^{1 / 4}\right)\right)_{i \in I}$ s.t

$$
\begin{gathered}
x_{i} \in \Omega, \forall i \in I, \\
B\left(x_{i}, \varepsilon^{1 / 4} / 4\right) \cap B\left(x_{i}, \varepsilon^{1 / 4} / 4\right)=\emptyset \text { if } i \neq j, \\
\cup_{i \in I} B\left(x_{i}, \varepsilon^{1 / 4}\right) \supset \Omega .
\end{gathered}
$$

Then we select discs (using Lemma 19) and we define

Notation 22. The initial good/bad discs

- Let $C_{0}=C_{0}(1 / 4,7 / 8), \varepsilon_{0}=\varepsilon_{0}(1 / 4,7 / 8)$ be defined by Lemma 19]2. For $\varepsilon<\varepsilon_{0}$, we say that $B\left(x_{i}, \varepsilon^{1 / 4}\right)$ is an initial good disc if

$$
F_{\varepsilon}\left(v_{\varepsilon}, B\left(x_{i}, \varepsilon^{1 / 4}\right) \cap \Omega\right) \leq C_{0}|\ln \varepsilon|
$$

and $B\left(x_{i}, \varepsilon^{1 / 4}\right)$ is an initial bad disc if

$$
F_{\varepsilon}\left(v_{\varepsilon}, B\left(x_{i}, \varepsilon^{1 / 4}\right) \cap \Omega\right)>C_{0}|\ln \varepsilon| .
$$

- We let $J=J(\varepsilon):=\left\{i \in I \mid B\left(x_{i}, \varepsilon^{1 / 4}\right)\right.$ is an initial bad disc $\}$.

An easy consequence of Lemma 18 is

Lemma 23. The number of initial bad discs is bounded

There is an integer $N$ which depends only on $g$ and $\Omega$ s.t.

$$
\text { Card } J \leq N
$$

Proof. Since each point of $\Omega$ is covered by at most $C>0$ (universal constant) discs $B\left(x_{i}, \varepsilon^{1 / 4}\right)$, we have

$$
\sum_{i \in J} F_{\varepsilon}\left(v_{\varepsilon}, B\left(x_{i}, \varepsilon^{1 / 4}\right) \cap \Omega\right) \leq C F_{\varepsilon}\left(v_{\varepsilon}, \Omega\right) .
$$

The previous assertion implies that Card $J \leq \frac{C \pi d}{C_{0}}+1$.

Let $\rho(\varepsilon)=\rho \downarrow 0$ be s.t.

$$
\frac{\rho}{\lambda \delta^{P+1}} \rightarrow 0 \text { and } \frac{|\ln \rho|^{3}}{|\ln \varepsilon|} \rightarrow 0
$$

Note that from Assumption (1.3), such a $\rho$ exists, e.g., $\rho=(\lambda \delta)^{P+2}$. (Recall that if the pinning term is periodic then $P=1$ )

The following result is a straightforward variant of Theorem IV.1 in [4]. 
Lemma 24. Separation of the initial bad discs

Let $\varepsilon_{n} \downarrow 0$. Then (possibly after passing to a subsequence and relabeling the indices), we may choose $J^{\prime} \subset J$ and a constant $\kappa$ independent of $n$ s.t.

$$
\begin{gathered}
J^{\prime}=\left\{1, \ldots, N^{\prime}\right\}, N^{\prime}=\mathrm{Cst}, \\
\left|x_{i}-x_{j}\right| \geq 16 \kappa \rho \text { for } i, j \in J^{\prime}, i \neq j,
\end{gathered}
$$

and

$$
\cup_{i \in J} B\left(x_{i}, \varepsilon_{n}^{1 / 4}\right) \subset \cup_{i \in J^{\prime}} B\left(x_{i}, \kappa \rho\right) .
$$

Notation 25. The $\rho$-bad disc

For $i \in J^{\prime}$, we say that $B\left(x_{i}, 2 \kappa \rho\right)$ is a $\rho$-bad disc.

Proposition 26. We have

1. $\frac{\rho}{\operatorname{dist}\left(B\left(x_{i}, 2 \kappa \rho\right), \partial \Omega\right)} \rightarrow 0$,

2. $\operatorname{deg}_{\partial B\left(x_{i}, 2 \kappa \rho\right)}\left(v_{\varepsilon_{n}}\right)>0$,

3. $F_{\varepsilon_{n}}\left(v_{\varepsilon_{n}}, B\left(x_{i}, 2 \kappa \rho\right)\right) \geq \pi b^{2} \operatorname{deg}_{\partial B\left(x_{i}, 2 \kappa \rho\right)}\left(v_{\varepsilon_{n}}\right) \ln \frac{\rho}{\varepsilon_{n}}-\mathcal{O}(1)$,

4. $\left|v_{\varepsilon_{n}}\right| \geq 1-C \sqrt{\frac{|\ln \rho|}{\left|\ln \varepsilon_{n}\right|}}$ in $\Omega \backslash \cup_{i \in J^{\prime}} \overline{B\left(x_{i}, 2 \kappa \rho\right)}$.

Proof. We prove Assertions 1., 2. and 3.. Set

$$
J_{0}^{\prime}:=\left\{i \in J^{\prime} \mid \operatorname{deg}_{\partial\left(B\left(x_{i}, 2 \kappa \rho\right) \cap \Omega\right)}\left(v_{\varepsilon_{n}}\right)>0\right\} .
$$

Since $\left|v_{\varepsilon_{n}}\right| \geq \frac{7}{8}$ in $\Omega \backslash \cup_{i \in J^{\prime}} \overline{B\left(x_{i}, 2 \kappa \rho\right)}$, we have

$$
0<d=\sum_{I \in J^{\prime}} \operatorname{deg}_{\partial\left(B\left(x_{i}, 2 \kappa \rho\right) \cap \Omega\right)}\left(v_{\varepsilon_{n}}\right) \leq \sum_{I \in J_{0}^{\prime}} \operatorname{deg}_{\partial\left(B\left(x_{i}, 2 \kappa \rho\right) \cap \Omega\right)}\left(v_{\varepsilon_{n}}\right) .
$$

Consequently $J_{0}^{\prime} \neq \emptyset$.

Up to a subsequence, we may assume that $J_{0}^{\prime}$ is independent of $n$.

From Proposition 20, for all $i \in J_{0}^{\prime}$, we have $\operatorname{dist}\left(B\left(x_{i}, \varepsilon^{1 / 4}\right), \partial \Omega\right) \gtrsim \delta$ (or $\delta^{P}$ if the pinning term is not periodic). Consequently, for $i \in J_{0}^{\prime}$ we find

$$
\frac{\operatorname{dist}\left(B\left(x_{i}, 2 \kappa \rho\right), \partial \Omega\right)}{\rho} \rightarrow+\infty
$$

since $\frac{\rho}{\lambda \delta^{P+1}} \rightarrow 0$.

Assertions 1., 2. and 3. will follow from the estimate

$$
F_{\varepsilon_{n}}\left(v_{\varepsilon_{n}}, B\left(x_{i}, 2 \kappa \rho\right)\right) \geq b^{2} \pi \operatorname{deg}_{\partial B\left(x_{i}, 2 \kappa \rho\right)}\left(v_{\varepsilon_{n}}\right) \ln \frac{\rho}{\varepsilon_{n}}-\mathcal{O}(1)
$$

valid for $i \in J_{0}^{\prime}$. Indeed, assume for the moment that (4.11) holds for $i \in J_{0}^{\prime}$.

Then, by combining (4.1), (4.2), (4.7), (4.8), (4.9) and (4.11), we find that $J_{0}^{\prime}=J^{\prime}$, i.e., 2. holds. Consequently, by combining Assertion 2. with (4.10), Assertion 1. yields and from Assertion 2. and (4.11), Assertion 3. holds. 
We now turn to the proof of (4.11), which relies on Proposition 4.1 in [20. We apply this proposition in the domain $B=B(0,2 \kappa)$, to the function $v^{\prime}(x)=v_{\varepsilon_{n}}\left[\rho\left(x-x_{i}\right)\right]$ and with the rescaled parameter $\xi_{\text {meso }}=\frac{\varepsilon}{\rho}$.

Note that, from (4.8), $\varepsilon \ll \xi_{\text {meso }} \ll \rho \ll \lambda \delta^{P+1}$ and $|\ln \varepsilon| \sim\left|\ln \xi_{\text {meso }}\right| \gg|\ln (\lambda \delta)|$.

Clearly, $v^{\prime}$ satisfies

$$
\begin{aligned}
\int_{B}\left\{\left|\nabla v^{\prime}\right|^{2}+\frac{1}{\xi_{\text {meso }}^{2}}\left(1-\left|v^{\prime}\right|^{2}\right)^{2}\right\} & =\int_{B\left(x_{i}, 2 \kappa \rho\right)}\left\{\left|\nabla v_{\varepsilon_{n}}\right|^{2}+\frac{1}{\varepsilon^{2}}\left(1-\left|v_{\varepsilon_{n}}\right|^{2}\right)^{2}\right\} \\
& =\mathcal{O}(|\ln \varepsilon|)=\mathcal{O}\left(\left|\ln \xi_{\text {meso }}\right|\right) .
\end{aligned}
$$

Hence, one may apply the following result of Sandier and Serfaty: there is $\left(B_{j}\right)_{j \in I}$, a finite covering of

$$
\left\{x \in B\left(0,2 \kappa-\xi_{\text {meso }} / b\right)|| v^{\prime}(x) \mid \leq 1-\left(\xi_{\text {meso }} / b\right)^{1 / 8}\right\}
$$

with disjoint balls $B_{j}$ of radius $r_{j}<10^{-3}$ s.t.

$$
\frac{1}{2} \int_{B \cap \cup B_{j}}\left\{\left|\nabla v^{\prime}\right|^{2}+\frac{b^{2}}{\xi_{\text {meso }}^{2}}\left(1-\left|v^{\prime}\right|^{2}\right)^{2}\right\} \geq \pi \sum_{j} d_{j}\left|\ln \xi_{\text {meso }}\right|-\mathcal{O}(1)
$$

here $d_{j}=\left\{\begin{array}{ll}\left|\operatorname{deg}_{\partial B_{j}}\left(v^{\prime}\right)\right| & \text { if } B_{j} \subset B\left(0,2 \kappa-\xi_{\text {meso }} / b\right) \\ 0 & \text { otherwise }\end{array}\right.$.

Note that from construction, $\left\{\left|v_{\varepsilon_{n}}\right| \leq 7 / 8\right\} \subset \cup_{J} B\left(x_{i}, \varepsilon_{n}^{1 / 4}\right) \subset \cup_{J^{\prime}} B\left(x_{i}, \kappa \rho\right)$. Consequently:

$$
\text { if } \operatorname{deg}_{\partial\left(B_{j} \cap B\left(0,2 \kappa-\xi_{\text {meso }} / b\right)\right)}\left(v^{\prime}\right) \neq 0 \text {, then we have } B_{j} \subset B\left(0, \frac{3}{2} \kappa\right) \text {. }
$$

Therefore, $\sum d_{j}=\operatorname{deg}_{\partial B(0,2 \kappa)}\left(v^{\prime}\right)=\operatorname{deg}_{\partial B\left(x_{i}, 2 \kappa \rho\right)}\left(v_{\varepsilon_{n}}\right)$ and

$$
\begin{aligned}
\frac{1}{2} \int_{B\left(x_{i}, 2 \kappa \rho\right)}\left\{\left|\nabla v_{\varepsilon_{n}}\right|^{2}+\frac{1}{2 \varepsilon^{2}}\left(1-\left|v_{\varepsilon_{n}}\right|^{2}\right)^{2}\right\} & \geq \pi \operatorname{deg}_{\partial B\left(x_{i}, 2 \kappa \rho\right)}\left(v_{\varepsilon_{n}}\right)\left|\ln \xi_{\text {meso }}\right|-\mathcal{O}(1) \\
& =\pi \operatorname{deg}_{\partial B\left(x_{i}, 2 \kappa \rho\right)}\left(v_{\varepsilon_{n}}\right) \ln \frac{\rho}{\varepsilon}-\mathcal{O}(1) .
\end{aligned}
$$

Thus (4.11) holds.

The last assertion is obtained using Lemmas 18 \& 19. Indeed, note that the proof of (4.11) gives a more precise result

$$
F_{\varepsilon_{n}}\left(v_{\varepsilon_{n}}, B\left(x_{i}, \frac{3}{2} \kappa \rho\right)\right) \geq b^{2} \pi \operatorname{deg}_{\partial B\left(x_{i}, 2 \kappa \rho\right)}\left(v_{\varepsilon_{n}}\right) \ln \frac{\rho}{\varepsilon_{n}}-\mathcal{O}(1)
$$

Let $x \in \Omega \backslash \cup_{J^{\prime}} B\left(x_{i}, 2 \kappa \rho\right)$ then $B\left(x, \varepsilon_{n}^{1 / 4}\right) \cap B\left(x_{i}, \frac{3}{2} \kappa \rho\right)=\emptyset$. Consequently, using Lemma 18 and the previous lower bound, we obtain:

$$
F_{\varepsilon_{n}}\left(v_{\varepsilon_{n}}, B\left(x, \varepsilon_{n}^{1 / 4}\right)\right) \leq I_{2 \kappa \rho, \varepsilon_{n}}+C_{0} \leq \pi d|\ln \rho|+C_{0} .
$$

Therefore, from Lemma 19, there is $C>0$, independent of $x$ s.t. $\left|v_{\varepsilon_{n}}(x)\right| \geq 1-C \sqrt{\frac{|\ln \rho|}{\left|\ln \varepsilon_{n}\right|}}$. 


\subsubsection{Location and degree of bad discs}

Let $w_{n}=\frac{v_{\varepsilon_{n}}}{\left|v_{\varepsilon_{n}}\right|} \in H^{1}\left(\Omega \backslash \cup_{J^{\prime}} \overline{B\left(x_{i}, 2 \kappa \rho\right)}, \mathbb{S}^{1}\right)$.

Proposition 27. The map $w_{n}$ is an almost minimal function for $I_{2 \kappa \rho, \varepsilon_{n}}$.

Proof. Indeed, denote $K_{n}=\frac{1}{2} \int_{\Omega \backslash \cup_{J^{\prime}} \overline{B\left(x_{i}, 2 \kappa \rho\right)}} U_{\varepsilon_{n}}^{2}\left|\nabla w_{n}\right|^{2}$, then we have

$$
\begin{aligned}
& K_{n} \leq F_{\varepsilon_{n}}\left(v_{\varepsilon_{n}}, \Omega \backslash \cup_{J^{\prime}} \overline{B\left(x_{i}, 2 \kappa \rho\right)}\right)+\int_{\Omega \backslash \cup_{J^{\prime}} \overline{B\left(x_{i}, 2 \kappa \rho\right)}} U_{\varepsilon_{n}}^{2}\left(1-\left|v_{\varepsilon_{n}}\right|^{2}\right)\left|\nabla w_{n}\right|^{2} \\
& =F_{\varepsilon_{n}}\left(v_{\varepsilon_{n}}, \Omega\right)-F_{\varepsilon_{n}}\left(v_{\varepsilon_{n}}, \cup_{J^{\prime}} B\left(x_{i}, 2 \kappa \rho\right)\right)+\int_{\Omega \backslash \cup_{J^{\prime}} \overline{B\left(x_{i}, 2 \kappa \rho\right)}} U_{\varepsilon_{n}}^{2}\left(1-\left|v_{\varepsilon_{n}}\right|^{2}\right)\left|\nabla w_{n}\right|^{2} \\
& \leq \text { (4.1), } \text { Prop 26] } \leq I_{2 \kappa \rho, \varepsilon_{n}}+C \sqrt{\frac{|\ln \rho|}{\left|\ln \varepsilon_{n}\right|}} \int_{\Omega \backslash \cup_{J^{\prime}} \overline{B\left(x_{i}, 2 \kappa \rho\right)}} U_{\varepsilon_{n}}^{2}\left|\nabla w_{n}\right|^{2}+\mathcal{O}(1) \\
& \leq \text { (4.1), Prop 26 } \leq I_{2 \kappa \rho, \varepsilon_{n}}+C \sqrt{\frac{|\ln \rho|}{\left|\ln \varepsilon_{n}\right|}} F_{\varepsilon_{n}}\left(v_{\varepsilon_{n}}, \Omega \backslash \cup_{J^{\prime}} \overline{B\left(x_{i}, 2 \kappa \rho\right)}\right)+\mathcal{O}(1) \\
& \leq \frac{\text { (4.1) }}{\text { Prop }(26.2)} \leq I_{2 \kappa \rho, \varepsilon_{n}}+C \sqrt{\frac{|\ln \rho|^{3}}{\left|\ln \varepsilon_{n}\right|}}+\mathcal{O}(1) \\
& \leq(4.8) \leq \quad I_{2 \kappa \rho, \varepsilon_{n}}+\mathcal{O}(1) \text {. }
\end{aligned}
$$

Remark 28. Note that the penultimate line in the proof of Proposition 27 is the main use of (1.3) (which is express in (4.8)).

By combining Proposition 12 with Proposition 27 in the periodic case or Proposition 17 if the pinning term is not periodic, we obtain the following

Corollary 29. The configuration $\left\{\left(x_{1}, \ldots, x_{N^{\prime}}\right),\left(\operatorname{deg}_{\partial B\left(x_{1}, 2 \kappa \rho\right)}\left(v_{\varepsilon_{n}}\right), \ldots, \operatorname{deg}_{\partial B\left(x_{N^{\prime}}, 2 \kappa \rho\right)}\left(v_{\varepsilon_{n}}\right)\right)\right\}$ is an almost minimal configuration of $I_{2 \kappa \rho, \varepsilon_{n}}$ and consequently, $N^{\prime}=d, \operatorname{deg}_{\partial B\left(x_{i}, 2 \kappa \rho\right)}\left(v_{\varepsilon_{n}}\right)=$ 1 for all $i$ and there is $\eta_{0}>0$ independent of large $n$ s.t.

$$
\begin{gathered}
\min \left\{\min _{i \neq j}\left|x_{i}-x_{j}\right|, \min _{i} \operatorname{dist}\left(x_{i}, \partial \Omega\right)\right\}>2 \eta_{0}, \\
B\left(x_{i}, 2 \eta_{0} \lambda \delta\right) \subset \omega_{\varepsilon} .
\end{gathered}
$$

\section{3 $H_{\text {loc }}^{1}$-weak convergence}

In order to keep notations simple, we replace from now on, $2 \kappa \rho$ by $\rho / 2$.

Using Corollary 29 , there is $\left\{a_{1}, \ldots, a_{d}\right\} \subset \Omega$ s.t. possibly after passing to a subsequence, we have $x_{i}^{n}=x_{i} \rightarrow a_{i}$.

Let $\rho_{0}>0$ be defined as

$$
\rho_{0}=10^{-2} \cdot \min _{k \neq l}\left\{\operatorname{dist}\left(a_{k}, \partial \Omega\right),\left|a_{k}-a_{l}\right|\right\} .
$$




\subsubsection{The contribution of the modulus is bounded in the whole domain}

We are now in position to bound the potential part of $F_{\varepsilon}\left(v_{\varepsilon}\right)$. More precisely we have

Proposition 30. We have $\int_{\Omega}\left\{\left.|\nabla| v_{\varepsilon_{n}}\right|^{2}+\frac{1}{\varepsilon_{n}^{2}}\left(1-\left|v_{\varepsilon_{n}}\right|^{2}\right)^{2}\right\}=\mathcal{O}(1)$.

Proof. From (4.1), Proposition 26 (Assertion 1., 2. and 3.) and Proposition 27, we infer that

$$
\int_{\Omega \backslash \cup_{i} \overline{B\left(x_{i}, \rho / 2\right)}}\left\{\left.|\nabla| v_{\varepsilon_{n}}\right|^{2}+\frac{1}{\varepsilon_{n}^{2}}\left(1-\left|v_{\varepsilon_{n}}\right|^{2}\right)^{2}\right\}=\mathcal{O}(1) .
$$

Consequently it suffices to obtain a similar estimate in $B\left(x_{i}, \rho / 2\right)$. Note that $B\left(x_{i}, \rho\right) \subset \omega_{\varepsilon}$. Thus, if we set

$$
u^{\prime}(x)=\frac{u_{\varepsilon_{n}}\left(x_{i}+\rho x\right)}{b}: B(0,1) \rightarrow \mathbb{C},
$$

then $u^{\prime}$ solves

$$
-\Delta u^{\prime}=\frac{1}{\left[\varepsilon_{n} /(b \rho)\right]^{2}} u^{\prime}\left(1-\left|u^{\prime}\right|^{2}\right) \text { in } B(0,1)
$$

From [5], we obtain

$$
\frac{1}{2} \int_{B(0,1 / 2)}\left\{|\nabla| u^{\prime}||^{2}+\frac{b^{2} \rho^{2}}{2 \varepsilon_{n}^{2}}\left(1-\left|u^{\prime}\right|^{2}\right)^{2}\right\}=\mathcal{O}(1) .
$$

This estimate is the subject of Theorem 1 for the potential part and Proposition 1 in [5] for the gradient of the modulus (see also Corollary 1 in [5]).

$$
\begin{aligned}
& \text { Set } K_{n}=\frac{1}{2} \int_{B(0,1 / 2)}\left\{|\nabla| u^{\prime}||^{2}+\frac{b^{2} \rho^{2}}{2 \varepsilon_{n}^{2}}\left(1-\left|u^{\prime}\right|^{2}\right)^{2}\right\} \text {. Using Proposition 3, we obtain } \\
& \begin{aligned}
K_{n}=\mathcal{O}(1) & =\frac{1}{2 b^{2}} \int_{B\left(x_{i}, \rho / 2\right)}\left\{\left.|\nabla| U_{\varepsilon_{n}} v_{\varepsilon_{n}}\right|^{2}+\frac{b^{4}}{2 \varepsilon_{n}^{2}}\left(1-\frac{\left|U_{\varepsilon_{n}} v_{\varepsilon_{n}}\right|^{2}}{b^{2}}\right)^{2}\right\} \\
& =\frac{1}{2} \int_{B\left(x_{i}, \rho / 2\right)}\left\{\left.|\nabla| v_{\varepsilon_{n}}\right|^{2}+\frac{b^{2}}{2 \varepsilon_{n}^{2}}\left(1-\left|v_{\varepsilon_{n}}\right|^{2}\right)^{2}\right\}+o_{n}(1) .
\end{aligned}
\end{aligned}
$$

Consequently, Proposition 30 holds.

\subsubsection{We bound the energy in a fixed perforated domain}

Proposition 31. For $0<\eta \leq \rho_{0}$, there is $C(\eta)>0$ independent of $n$ s.t. we have

$$
\frac{1}{2} \int_{\Omega \backslash \cup \overline{B\left(a_{i}, \eta\right)}}\left|\nabla v_{\varepsilon_{n}}\right|^{2} \leq C(\eta)
$$

Proof. We argue by contradiction and we assume that there is $\eta>0$ s.t., up to pass to a subsequence, we have $\int_{\Omega \backslash \cup \overline{B\left(a_{i}, \eta\right)}}\left|\nabla v_{\varepsilon_{n}}\right|^{2} \rightarrow \infty$.

Because $\int_{\Omega \backslash \cup \overline{B\left(a_{i}, \eta\right)}}\left|\nabla v_{\varepsilon_{n}}\right|^{2}=\int_{\Omega \backslash \cup \overline{B\left(a_{i}, \eta\right)}}\left|v_{\varepsilon_{n}}\right|^{2}\left|\nabla w_{n}\right|^{2}+\left|\nabla\left(\left|v_{\varepsilon_{n}}\right|\right)\right|^{2}$, from Propositions $26 \& 30$ we get $\int_{\Omega \backslash \cup \overline{B\left(a_{i}, \eta\right)}}\left|\nabla w_{n}\right|^{2} \rightarrow \infty$. Therefore, we have $\int_{\Omega \backslash \cup \overline{B\left(x_{i}, 10^{-1} \eta\right)}}\left|\nabla w_{n}\right|^{2} \rightarrow \infty$.

It is clear that we may get a map $\tilde{w}_{n} \in \mathcal{J}_{10^{-1} \eta, \varepsilon_{n}}\left(\mathbf{x}_{\varepsilon_{n}}, \mathbf{1}\right)$ s.t. $\int_{\Omega \backslash \cup \overline{B\left(x_{i}, 10^{-1} \eta\right)}}\left|\nabla \tilde{w}_{n}\right|^{2} \leq$ $C(\eta)$. 
For $i=1, \ldots, d$, using Proposition (45) (Appendix C], Section C.3. Page 44), we get the existence of a map $\tilde{w}_{i, n} \in H^{1}\left(B\left(x_{i}, 10^{-1} \eta\right) \backslash \overline{B\left(x_{i}, \rho / 2\right)}, \mathbb{S}^{1}\right)$ s.t. $\tilde{w}_{i, n}\left(x_{i}+10^{-1} \eta \mathrm{e}^{\imath \theta}\right)=$ $\tilde{w}_{n}\left(x_{i}+10^{-1} \eta \mathrm{e}^{\imath \theta}\right)$ and

$$
\int_{B\left(x_{i}, 10^{-1} \eta\right) \backslash \overline{B\left(x_{i}, \rho / 2\right)}} U_{\varepsilon_{n}}^{2}\left|\nabla \tilde{w}_{i, n}\right|^{2} \leq \int_{B\left(x_{i}, 10^{-1} \eta\right) \backslash \frac{B\left(x_{i}, \rho / 2\right)}{\varepsilon_{n}}} U^{2}\left|\nabla w_{n}\right|^{2}+\mathcal{O}(1)
$$

Therefore by extending $\tilde{w}_{n}$ with $\tilde{w}_{i, n}$ in $B\left(x_{i}, 10^{-1} \eta\right) \backslash \overline{B\left(x_{i}, \rho / 2\right)}$ we get a map still denoted $\tilde{w}_{n} \in H_{g}^{1}\left(\Omega \backslash \cup \overline{B\left(x_{i}, \rho / 2\right)}, \mathbb{S}^{1}\right)$ s.t.

$$
\frac{1}{2} \int_{\Omega \backslash \cup \overline{B\left(x_{i}, \rho / 2\right)}}\left|\nabla w_{n}\right|^{2}-\frac{1}{2} \int_{\Omega \backslash \cup \overline{B\left(x_{i}, \rho / 2\right)}}\left|\nabla \tilde{w}_{n}\right|^{2} \rightarrow \infty
$$

which is in contradiction with Proposition 27.

Consequently, there is $v_{*} \in H_{\text {loc }}^{1}\left(\bar{\Omega} \backslash\left\{a_{1}, \ldots, a_{d}\right\}, \mathbb{S}^{1}\right)$ s.t., up to pass to a subsequence, $v_{\varepsilon_{n}} \rightarrow v_{*}$ in $H_{\text {loc }}^{1}\left(\bar{\Omega} \backslash\left\{a_{1}, \ldots, a_{d}\right\}\right)$. Next section is dedicate to the limiting equation of $v_{*}$.

\subsubsection{We establish the limiting equation}

In order to obtain the expression of the homogenized problem, we use the unfolding operator (see [8], definition 2.1).

The use of the unfolding operator needs a slightly modification of the cell period. More precisely, instead of considering the $\delta \times \delta$-grid whose vertices-grid are the points $\{\delta(k, l)+(1 / 2,1 / 2) \mid k, l \in \mathbb{Z}\}$, we consider the one whose vertices-grid are $\{\delta(k, l) \mid k, l \in \mathbb{Z}\}$.

Thus instead of having cells which contain one inclusion at their center we have cells with quarters of inclusion at their vertices. (See Figure 3 )

More specifically, we define, for $\Omega_{0} \subset \mathbb{R}^{2}$ an open set, $p \in(1, \infty)$ and $\delta>0$,

$$
\begin{aligned}
\mathcal{T}_{\delta}: L^{p}\left(\Omega_{0}\right) & \rightarrow \\
\phi & \mapsto \mathcal{T}_{\delta}(\phi)(x, y)=\left\{\begin{array}{cl}
L^{p}\left(\Omega_{0} \times \tilde{Y}\right) \\
\phi\left(\delta\left[\frac{x}{\delta}\right]+\delta y\right) & \text { for }(x, y) \in \tilde{\Omega}_{\delta}^{\text {incl }} \times \tilde{Y} \\
0 & \text { for }(x, y) \in \Lambda_{\delta} \times \tilde{Y}
\end{array} .\right.
\end{aligned}
$$

Here, $\tilde{Y}=(0,1)^{2},[s]$ is the integer part of $s \in \mathbb{R}$ and

$$
\tilde{\Omega}_{\delta}^{\text {incl }}:=\bigcup_{\substack{\tilde{Y}_{\delta}^{K} \subset \Omega_{0}, K \in \mathbb{Z}^{2} \\ \tilde{Y}_{\delta}^{K}=\delta \cdot(K+\tilde{Y})}} \overline{\tilde{Y}_{\delta}^{K}}, \Lambda_{\delta}:=\Omega_{0} \backslash \tilde{\Omega}_{\delta}^{\text {incl }} \text { and }\left[\frac{x}{\delta}\right]:=\left(\left[\frac{x_{1}}{\delta}\right],\left[\frac{x_{2}}{\delta}\right]\right) .
$$

An adaptation of a result of Sauvageot ([21], Theorem 4) gives the following

Proposition 32. Let $\Omega_{0} \subset \mathbb{R}^{2}$ be a smooth bounded open set. Let $v_{n} \in H^{2}\left(\Omega_{0}, \mathbb{C}\right)$ be s.t.

1. $\left|v_{n}\right| \leq 1$ and $\int_{\Omega_{0}}\left(1-\left|v_{n}\right|^{2}\right)^{2} \rightarrow 0$

2. $v_{n} \rightarrow v_{*}$ in $H^{1}\left(\Omega_{0}\right)$ for some $v_{*} \in H^{1}\left(\Omega_{0}, \mathbb{S}^{1}\right)$,

3. there are $H_{n} \in W^{1, \infty}\left(\Omega_{0},\left[b^{2}, 1\right]\right)$ and $\delta=\delta_{n} \downarrow 0$ s.t. $\mathcal{T}_{\delta}\left(H_{n}\right)(x, y) \rightarrow H_{0}(y)$ in $L^{2}\left(\Omega_{0} \times \tilde{Y}\right)$, 


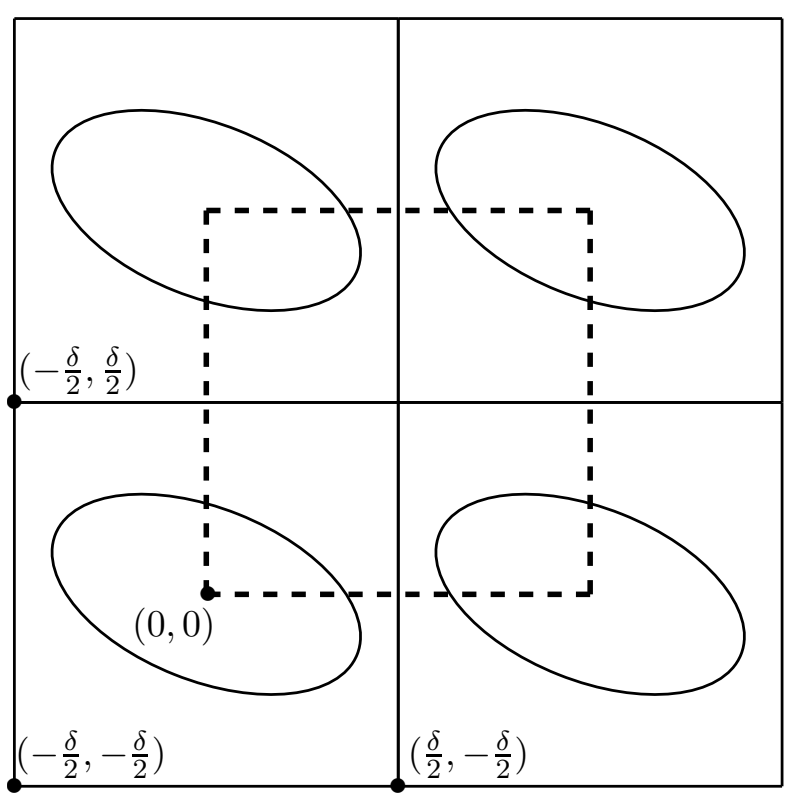

(a) Four period-cells which are obtained from $Y=$ $(-1 / 2,1 / 2)^{2}$ and the new period-cell in dash obtained from $\tilde{Y}=(0,1)^{2}$

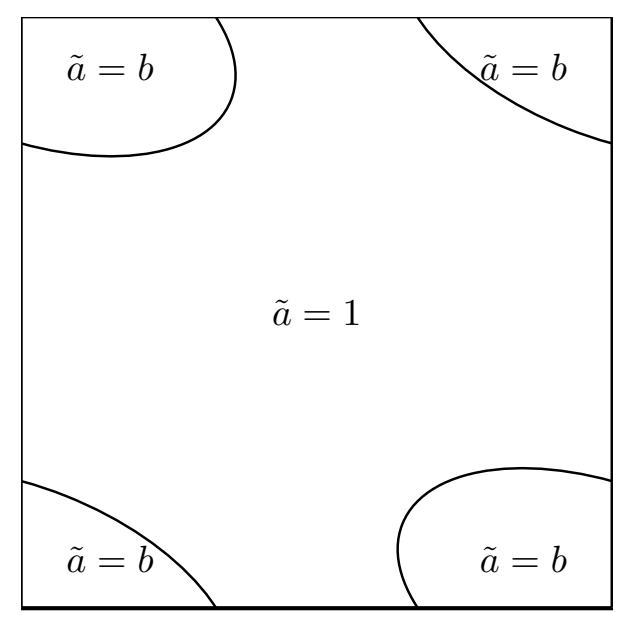

(b) The new unit-cell $\tilde{Y}$ with four quarters of an inclusion and the values of $\tilde{a}=a_{\mid \tilde{Y}}^{\lambda}$

Figure 3: The modification of the reference cell

$$
\text { 4. }-\operatorname{div}\left(H_{n} \nabla v_{n}\right)=v_{n} f_{n}(x), f_{n} \in L^{\infty}\left(\Omega_{0}, \mathbb{R}\right) .
$$

Then $v_{*}$ is a solution of

$$
-\operatorname{div}\left(\mathcal{A} \nabla v_{*}\right)=\left(\mathcal{A} \nabla v_{*} \cdot \nabla v_{*}\right) v_{*}
$$

where $\mathcal{A}$ is the homogenized matrix of $H_{0}(\dot{\bar{\delta}}) \operatorname{Id}_{\mathbb{R}^{2}}$. (See Appendix $\mathbb{E}$ to have more details about $\mathcal{A}$ )

The proof of Proposition 32 is postponed to Appendix $\mathrm{F}$.

We apply the above proposition with $\Omega_{0}=\Omega \backslash \cup \overline{B\left(a_{i}, \eta\right)}, \delta=\delta_{n} \downarrow 0$ the sequence which defines $a_{\varepsilon_{n}}$ and $H_{n}=U_{\varepsilon_{n}}^{2}$. By application of Proposition 3, we obtain

$$
\mathcal{T}_{\delta}\left(U_{\varepsilon_{n}}^{2}\right)(x, y) \stackrel{L^{2}\left(\Omega_{0} \times \tilde{Y}\right)}{\rightarrow}\left\{\begin{array}{ll}
\tilde{a}^{2}(y) & \text { if } \lambda \equiv 1 \\
1 & \text { if } \lambda \rightarrow 0
\end{array} .\right.
$$

Note that the $\tilde{Y}$-periodic extension of $\tilde{a}$ in $\mathbb{R}^{2}$ is equal to the $Y$-periodic extension of $1-\left(1-b^{2}\right) \mathbb{I}_{\omega^{\lambda}}$ which is $a^{\lambda}$ (defined Construction 1).

We find that $v_{*}$ solves

$$
\begin{aligned}
-\operatorname{div}\left(\mathcal{A} \nabla v_{*}\right) & =\left(\mathcal{A} \nabla v_{*} \cdot \nabla v_{*}\right) v_{*} & & \text { if } \lambda \equiv 1 \\
-\Delta v_{*} & =\left|\nabla v_{*}\right|^{2} v_{*} & & \text { if } \lambda \rightarrow 0
\end{aligned} .
$$

Here $\mathcal{A}$ is the homogenized matrix of $\left[a^{\lambda}(\dot{\bar{\delta}})\right]^{2} \operatorname{Id}_{\mathbb{R}^{2}}$.

\subsection{The small bad discs}

\subsubsection{Definition}

With the help the bound on the potential part of the minimizers $\frac{1}{\varepsilon^{2}} \int_{\Omega}\left(1-\left|v_{\varepsilon}\right|^{2}\right)^{2} \leq C$ (Proposition 30), in the spirit of [4] (Theorem III.3), we may detect the vorticity defects 
(the connected components of $\left\{\left|v_{\varepsilon}\right| \leq 7 / 8\right\}$ ) by smaller discs (discs with radius of order $\varepsilon$ ) than the $\rho$-bad discs (Notation 25).

Notation 33. The small bad discs

The construction is done as follows:

- We consider a covering of $\Omega$ as in Notation 21 (page 21). We fix $\rho=\rho(\varepsilon) \downarrow 0$ s.t. Assumption (4.8) holds. For sufficiently small $\varepsilon$, we denote

$$
S_{\rho}(\varepsilon)=\{B(x, R / 2) \mid B(x, R) \text { given by Notation 25, page 22 }\} .
$$

- Following [4] (Theorem III.3), for $l \geq 2$, there are $\kappa_{l}, \mu_{l}>0$ (depending only on $\Omega, g$ and l) s.t. for $x \in \Omega$, if

then

$$
\frac{1}{\varepsilon^{2}} \int_{B\left(x, 2 \kappa_{l} \varepsilon\right)}\left(1-\left|v_{\varepsilon}\right|^{2}\right)^{2} \leq \mu_{l}
$$

$$
\left|v_{\varepsilon}\right| \geq 1-\frac{1}{l^{2}} \text { in } B\left(x, \kappa_{l} \varepsilon\right) .
$$

We fix $l \geq 2$ and we drop the subscript $l$. We now consider a covering of $\cup_{B \in S_{\rho}(\varepsilon)} B$ by $\operatorname{discs}\left(B\left(x_{i}^{\varepsilon}, \kappa \varepsilon\right)\right)_{i \in I}$ s.t

$$
\begin{gathered}
x_{i}^{\varepsilon} \in \cup_{B \in S_{\rho}(\varepsilon)} B, \forall i \in I, \\
B\left(x_{i}^{\varepsilon}, \kappa \varepsilon / 4\right) \cap B\left(x_{j}^{\varepsilon}, \kappa \varepsilon / 4\right)=\emptyset \text { if } i \neq j, \\
\cup_{i \in I} B\left(x_{i}^{\varepsilon}, \kappa \varepsilon\right) \supset \cup_{B \in S_{\rho}(\varepsilon)} B .
\end{gathered}
$$

We say that $B\left(x_{i}^{\varepsilon}, \kappa \varepsilon\right)$ is a small good disc if

$$
\frac{1}{\varepsilon^{2}} \int_{B\left(x_{i}^{\varepsilon}, 2 \kappa \varepsilon\right)}\left(1-\left|v_{\varepsilon}\right|^{2}\right)^{2}<\mu .
$$

- If $B\left(x_{i}^{\varepsilon}, \kappa \varepsilon\right)$ is not a small good disc, then we call it a small bad disc. We denote $J \subset I$ the set of indices of small bad discs.

Following [4], using Proposition [30, there is $N_{l}=N>0$ (depending only on $\Omega, g$ and l) s.t. $\operatorname{Card}(J) \leq N$.

Using a standard separation process (Lemma 42), for $\varepsilon_{n} \downarrow 0$, possibly after passing to a subsequence and relabeling the discs, there are $J^{\prime} \subset J$ and $\kappa^{\prime} \in\left\{\kappa, \ldots, 9^{N-1} \kappa\right\}$ s.t.

$$
\left\{\left|v_{\varepsilon_{n}}\right|<1-1 / l^{2}\right\} \subset \cup_{i \in J} B\left(x_{i}^{\varepsilon_{n}}, \kappa \varepsilon_{n}\right) \subset \cup_{i \in J^{\prime}} B\left(x_{i}^{\varepsilon_{n}}, \kappa^{\prime} \varepsilon_{n}\right)
$$

and

$$
\frac{\left|x_{i}^{\varepsilon_{n}}-x_{j}^{\varepsilon_{n}}\right|}{\varepsilon_{n}} \geq 8 \kappa^{\prime} \text { if } i, j \in J^{\prime}, i \neq j .
$$

By a standard iterative procedure, we may assume that the small bad discs are mutually far away in the $\varepsilon$-scale.

Proposition 34. Possibly after passing to a subsequence, we have, for large $R$ and $J^{\prime \prime} \subset J^{\prime}$,

$$
\left\{\left|v_{\varepsilon_{n}}\right|<1-1 / l^{2}\right\} \subset \cup_{i \in J^{\prime \prime}} B\left(x_{i}^{\varepsilon_{n}}, R \varepsilon_{n}\right),
$$

where, for $i \neq j$,

$$
\frac{\left|x_{i}^{\varepsilon_{n}}-x_{j}^{\varepsilon_{n}}\right|}{\varepsilon_{n}} \rightarrow \infty \text { as } n \rightarrow \infty .
$$

Notation 35. The small and separated bad discs

The discs $\left\{B\left(x_{i}^{\varepsilon_{n}}, R \varepsilon_{n}\right) \mid i \in J^{\prime \prime}\right\}$ obtained in Proposition 34 are the small and separated bad discs. 


\subsubsection{Each $\rho$-bad disc contains exactly one small bad disc}

By construction, we know that the small and separated bad discs (defined Notation 35) are covered by the $\rho$-bad discs defined Notation 25) (page 22). We next prove that there are exactly $d$ small bad discs and consequently, there is exactly one small bad discs per $\rho$-bad discs.

Proposition 36. For large $n$ and for all $i \in J^{\prime \prime}$, we have

$$
\operatorname{deg}_{\partial B\left(x_{i}^{\varepsilon_{n}}, R \varepsilon_{n}\right)}\left(v_{\varepsilon_{n}}\right)=1 .
$$

Proof. First we prove that, for large $n$ and for all $i$, we have

$$
\operatorname{deg}_{\partial B\left(x_{i}^{\varepsilon_{n}}, R \varepsilon_{n}\right)}\left(v_{\varepsilon_{n}}\right) \neq 0 .
$$

We argue by contradiction and we assume that, up to a subsequence, there is $i$ s.t. $\operatorname{deg}_{\partial B\left(x_{i}^{\varepsilon_{n}}, R \varepsilon_{n}\right)}\left(v_{\varepsilon_{n}}\right)=0$.

Set

$$
M_{n}=\min \left\{b \min _{i \neq j} \frac{\left|x_{i}^{\varepsilon_{n}}-x_{j}^{\varepsilon_{n}}\right|}{8 R \varepsilon_{n}}, \delta^{-1}\right\}
$$

and set

$$
\begin{aligned}
u_{n}^{\prime}: B\left(0, M_{n}\right) & \rightarrow \quad \\
x & \mapsto \frac{u_{\varepsilon_{n}}\left(\frac{\varepsilon_{n}}{b} x+x_{i}^{\varepsilon_{n}}\right)}{b} .
\end{aligned}
$$

Note that, $B\left(x_{i}^{\varepsilon_{n}}, M_{n} \varepsilon_{n}\right) \subset \omega_{\varepsilon_{n}}$ and by Proposition 34, we have $M_{n} \rightarrow \infty$.

It is easy to check that $u_{n}^{\prime}$ solves $-\Delta u_{n}^{\prime}=u_{n}^{\prime}\left(1-\left|u_{n}^{\prime}\right|^{2}\right)$. Following [7], up to a subsequence,

$$
u_{n}^{\prime} \rightarrow u_{0} \text { in } C_{\mathrm{loc}}^{2}\left(\mathbb{R}^{2}\right)
$$

here $u_{0}: \mathbb{R}^{2} \rightarrow \mathbb{C}$ solves $-\Delta u_{0}=u_{0}\left(1-\left|u_{0}\right|^{2}\right)$ in $\mathbb{R}^{2}$.

Then two cases occur: $\int_{\mathbb{R}^{2}}\left(1-\left|u_{0}\right|^{2}\right)^{2}<\infty$ or $\int_{\mathbb{R}^{2}}\left(1-\left|u_{0}\right|^{2}\right)^{2}=\infty$.

Assume first that $\int_{\mathbb{R}^{2}}\left(1-\left|u_{0}\right|^{2}\right)^{2}<\infty$. From [7], noting that the degree of $u_{0}$ on large circles centered in 0 is 0 , we obtain that $u_{0}=$ Cst $\in \mathbb{S}^{1}$ and consequently $\int_{\mathbb{R}^{2}}\left(1-\left|u_{0}\right|^{2}\right)^{2}=0$.

Since $u_{n}^{\prime} \rightarrow u_{0}$ in $L^{4}(B(0,2 b R))(R \geq \kappa)$, we find that

$$
\begin{aligned}
\int_{B(0,2 b R)}\left(1-\left|u_{n}^{\prime}\right|^{2}\right)^{2} & =\frac{b^{2}}{\varepsilon_{n}^{2}} \int_{B\left(x_{i}^{\varepsilon_{n}}, 2 R \varepsilon_{n}\right)}\left(1-\left|u_{n} / b\right|^{2}\right)^{2} \\
& =\frac{b^{2}}{\varepsilon_{n}^{2}} \int_{B\left(x_{i}^{\varepsilon_{n}}, 2 R \varepsilon_{n}\right)}\left(1-\left|v_{\varepsilon_{n}}\right|^{2}\right)^{2}+o_{n}(1) \rightarrow 0 .
\end{aligned}
$$

Noting that $B\left(x_{i}^{\varepsilon_{n}}, \kappa \varepsilon_{n}\right)$ is a small bad disc and that $B\left(x_{i}^{\varepsilon_{n}}, 2 \kappa \varepsilon_{n}\right) \subset B\left(x_{i}^{\varepsilon_{n}}, 2 R \varepsilon_{n}\right)$, we have a contradiction.

$$
\begin{aligned}
& \text { Therefore } \int_{\mathbb{R}^{2}}\left(1-\left|u_{0}\right|^{2}\right)^{2}=\infty \text {. Consequently, there is } M_{0}>0 \text { s.t. } \\
& \qquad \int_{B\left(0, b M_{0}\right)}\left(1-\left|u_{0}\right|^{2}\right)^{2} \geq \sup _{n}\left\{\frac{4 b^{2}}{\varepsilon_{n}^{2}} \int_{\Omega}\left(1-\left|v_{\varepsilon_{n}}\right|^{2}\right)^{2}\right\} .
\end{aligned}
$$


Thus, for large $n$ we have

$$
\begin{aligned}
\int_{B\left(0, b M_{n}\right)}\left(1-\left|u_{n}^{\prime}\right|^{2}\right)^{2} & =\frac{b^{2}}{\varepsilon_{n}^{2}} \int_{B\left(x_{i}^{\varepsilon_{n}}, M_{n} \varepsilon_{n}\right)}\left(1-\left|u_{\varepsilon_{n}} / b\right|^{2}\right)^{2} \\
& =\frac{b^{2}}{\varepsilon_{n}^{2}} \int_{B\left(x_{i}^{\varepsilon_{n}}, M_{n} \varepsilon_{n}\right)}\left(1-\left|v_{\varepsilon_{n}}\right|^{2}\right)^{2}+o_{n}(1) \\
& \geq \sup _{n}\left\{\frac{2 b^{2}}{\varepsilon_{n}^{2}} \int_{\Omega}\left(1-\left|v_{\varepsilon_{n}}\right|^{2}\right)^{2}\right\},
\end{aligned}
$$

which is a contradiction with $B\left(x_{i}^{\varepsilon_{n}}, M_{n} \varepsilon_{n}\right) \subset \Omega$.

Consequently we obtain that for large $n, \operatorname{deg}_{\partial B\left(x_{i}^{\varepsilon_{n}}, R \varepsilon_{n}\right)}\left(v_{\varepsilon_{n}}\right) \neq 0$.

Now we prove that

$$
\operatorname{deg}_{\partial B\left(x_{i}^{\varepsilon_{n}}, R \varepsilon_{n}\right)}\left(v_{\varepsilon_{n}}\right)=1 \text { for all } i \text { and large } n .
$$

Note that each small bad disc contains at least a zero of $v_{\varepsilon_{n}}$. Consequently, for $\rho$ satisfying (4.8), all small bad discs are included in a $\rho$-bad disc $B(y, \rho)$ defined Notation 25 (page 22). (For sake of simplicity we wrote $B(y, \rho)$ instead of $B(y, 2 \kappa \rho)$ ).

If $B(y, \rho)$ is a $\rho$-bad disc, we denote $\Lambda_{y}=\left\{i \in J^{\prime \prime} \mid x_{i}^{\varepsilon_{n}} \in B(y, \rho)\right\}$. Clearly, if $\operatorname{Card}\left(\Lambda_{y}\right)=1$, then (4.15) holds.

We define

$$
æ_{n}^{y}:=\left\{\begin{array}{ll}
10^{-2} \min _{i, j \in \Lambda_{y}, i \neq j}\left|x_{i}^{\varepsilon_{n}}-x_{j}^{\varepsilon_{n}}\right| & \text { if } \operatorname{Card}\left(\Lambda_{y}\right)>1 \\
R \varepsilon_{n} & \text { otherwise }
\end{array} .\right.
$$

From Proposition 34, if $\operatorname{Card}\left(\Lambda_{y}\right)>1$ then $æ_{n} / \varepsilon_{n} \rightarrow \infty$.

For simplicity, we assume that $y=0$ and we let

$$
\tilde{B}=B(0,8) \backslash \cup_{i \in \Lambda_{0}} \overline{B\left(\frac{x_{i}^{\varepsilon_{n}}}{\rho}, \frac{æ_{n}^{0}}{\rho}\right)} .
$$

Remark 37. Note that from Corollary 29 we have $B(y, 16 \rho) \subset \omega_{\varepsilon}$.

Clearly, we are in position to apply Theorem 2 in [14] in the perforated domain $\tilde{B}$. After scaling, we find that

$$
\frac{1}{2} \int_{B(y, 8 \rho) \backslash \cup \overline{B\left(x_{i}^{\varepsilon_{n}}, æ_{n}^{y}\right)}}\left|\nabla v_{\varepsilon_{n}}\right|^{2} \geq \pi\left|\sum_{i \in \Lambda_{y}} \operatorname{deg}_{\partial B\left(x_{i}^{\varepsilon_{n}}, R \varepsilon_{n}\right)}\left(v_{\varepsilon_{n}}\right)\right| \ln \frac{\rho}{æ_{n}^{y}}-C=\pi \ln \frac{\rho}{æ_{n}^{y}}-C .
$$

In order to prove (4.15), we observe the case where there is $y$ s.t. $\operatorname{Card}\left(\Lambda_{y}\right)>1$. Recall that if for all $y$ centers of $\rho$-bad discs we have $\operatorname{Card}\left(\Lambda_{y}\right)=1$, then (4.15) holds. Since $\operatorname{deg}_{\partial B\left(x_{i}^{\varepsilon_{n}}, R \varepsilon_{n}\right)}\left(v_{\varepsilon_{n}}\right) \neq 0$, if $\operatorname{Card}\left(\Lambda_{y}\right)>1$, then we have

$$
\sum_{i \in \Lambda_{y}}\left|\operatorname{deg}_{\partial B\left(x_{i}^{\varepsilon_{n}}, R \varepsilon_{n}\right)}\left(v_{\varepsilon_{n}}\right)\right|>1
$$

We obtain easily the following lower bound for $i \in \Lambda_{y}$ :

$$
\frac{1}{2} \int_{B\left(x_{i}^{\varepsilon_{n}}, æ_{n}^{y}\right) \backslash \frac{B\left(x_{i}^{\varepsilon_{n}}, R \varepsilon_{n}\right)}{}}\left|\nabla v_{\varepsilon_{n}}\right|^{2} \geq \pi\left|\operatorname{deg}_{\partial B\left(x_{i}^{\varepsilon_{n}}, R \varepsilon_{n}\right)}\left(v_{\varepsilon_{n}}\right)\right| \ln \frac{æ_{n}^{y}}{R \varepsilon_{n}}-C .
$$


Summing for $i \in \Lambda_{y}$, we obtain that

$$
\sum_{i \in \Lambda_{y}} \frac{1}{2} \int_{B\left(x_{i}^{\varepsilon_{n}}, æ_{n}^{y}\right) \backslash \frac{}{B\left(x_{i}^{\varepsilon_{n}}, R \varepsilon_{n}\right)}}\left|\nabla v_{\varepsilon_{n}}\right|^{2} \geq 2 \pi \ln \frac{æ_{n}^{y}}{R \varepsilon_{n}}-C .
$$

Consequently, we deduce that

$$
\sum_{y} \frac{1}{2} \int_{B(y, 8 \rho) \backslash \cup \frac{}{B\left(x_{i}^{\varepsilon n}, R \varepsilon_{n}\right)}}\left|\nabla v_{\varepsilon_{n}}\right|^{2} \geq \pi d \ln \frac{\rho}{R \varepsilon_{n}}+\pi \sum_{y \text { s.t. } \operatorname{Card}\left(\Lambda_{y}\right)>1} \ln \frac{æ_{n}^{y}}{R \varepsilon_{n}}-\mathcal{O}_{n}(1) .
$$

From Lemma 18 and Propositions 26 \& 27, we deduce easily

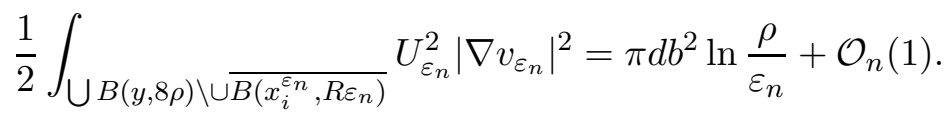

Combining the previous estimates, we obtain that

$$
\left\{y \text { center of } \rho \text {-bad } \operatorname{discs} \mid \operatorname{Card}\left(\Lambda_{y}\right)>1\right\}=\emptyset,
$$

and thus $\operatorname{deg}_{\partial B\left(x_{i}^{\varepsilon_{n}}, R \varepsilon_{n}\right)}\left(v_{\varepsilon_{n}}\right)=1$ for large $n$.

Corollary 38. For large $n$, there is a unique zero inside each small and separated bad discs defined Notation 35 (page 28).

Proof. From Proposition [36, one may assume that $v_{\varepsilon_{n}}\left(x_{i}^{\varepsilon_{n}}\right)=0$.

Let $i \in\{1, \ldots, d\}$. In view of (4.14), if we denote

$$
\begin{array}{cl}
u_{n}^{\prime}: B\left(0, M_{n}\right) & \rightarrow \\
x & \mapsto \frac{u_{\varepsilon_{n}}\left(\frac{\varepsilon_{n}}{b} x+x_{i}^{\varepsilon_{n}}\right)}{b},
\end{array}
$$

then, up to a subsequence,

$$
u_{n}^{\prime} \rightarrow u_{0} \text { in } C^{1}(\overline{B(0, b R)}) .
$$

Here $M_{n}$ is defined in (4.13).

Using the main result of [19], we have the existence of a universal function $f: \mathbb{R}^{+} \rightarrow$ $[0,1]$ s.t.

$$
\left\{\begin{array}{c}
u_{0}(x)=f(|x|) \mathrm{e}^{\imath\left(\theta+\theta_{i}\right)} \text { where } x=|x| \mathrm{e}^{\imath \theta}, \theta_{i} \in \mathbb{R} \\
\text { and } f: \mathbb{R}^{+} \rightarrow \mathbb{R}^{+} \text {is increasing. }
\end{array}\right.
$$

Therefore, we may apply Theorem 2.3 in [2] in order to obtain that, for large $n, u_{n}^{\prime}$ has a unique zero in $B(0, b R)$. Consequently, for large $n, v_{\varepsilon_{n}}$ has a unique zero in $B\left(x_{i}^{\varepsilon_{n}}, R \varepsilon_{n}\right)$.

Corollary 39. One may consider that $R$ depends only on $l$ ( $R$ is independent of the extraction we consider), i.e, for $l \geq 2$ there is $R_{l}>0$ s.t. for small $\varepsilon$, denoting $\left\{x_{i}^{\varepsilon} \mid i \in\right.$ $\{1, \ldots, d\}\}$ the set of zeros of a minimizer $v_{\varepsilon}$, we have

$$
\left\{\left|v_{\varepsilon}\right|<1-1 / l^{2}\right\} \subset \cup_{i} B\left(x_{i}^{\varepsilon}, R_{l} \varepsilon\right) .
$$


Proof. From Corollary 38, one may assume that $v_{\varepsilon_{n}}\left(x_{i}^{\varepsilon_{n}}\right)=0$.

Let $f: \mathbb{R}^{+} \rightarrow \mathbb{R}^{+}$be defined as in (4.18) and $u_{n}^{\prime}$ as in (4.16). For $l \geq 2$, consider $R_{l}>0$ s.t.

$$
l \mapsto R_{l} \text { is increasing and } f\left(b R_{l}\right) \geq 1-\frac{1}{2 l^{2}} .
$$

Note that from [22], one may consider $R_{l} \simeq \sqrt{2} l / b$.

By uniqueness of $f$, the full sequence $\left|u_{n}^{\prime}\right|$ converges to $f$ in $L^{\infty}\left[B\left(0, b \max \left\{R, R_{l}\right\}\right)\right]$. Consequently, for $n$ sufficiently large, since $f$ is not decreasing,

$$
\left\{\left|v_{\varepsilon_{n}}\right|<1-1 / l^{2}\right\} \subset \cup_{i} B\left(x_{i}^{\varepsilon_{n}}, R_{l} \varepsilon_{n}\right) .
$$

\subsection{Asymptotic expansion of $F_{\varepsilon}\left(v_{\varepsilon}\right)$}

This section is essentially devoted to proof Theorem 5, The key argument in this proof is Proposition 40.

\subsubsection{Statement of the main result and a corollary}

We state a technical and fundamental result and a direct corollary.

Proposition 40. For all $\varepsilon_{n} \downarrow 0$, up to a subsequence, there is $\rho=\rho\left(\varepsilon_{n}\right)$ s.t. $\varepsilon_{n} \ll \rho \ll$ $\lambda \delta^{3 / 2}$ and s.t. when $n \rightarrow \infty$ the following holds

$$
F_{\varepsilon_{n}}\left(v_{\varepsilon_{n}}\right) \geq J_{\rho, \varepsilon_{n}}+d b^{2}\left(\pi \ln \frac{b \rho}{\varepsilon_{n}}+\gamma\right)+o_{n}(1),
$$

where $J_{\rho, \varepsilon}$ is defined in (3.6) and $\gamma$ is the universal constant defined in [4], Lemma IX.1.

Corollary 41. Let $\varepsilon_{n} \downarrow 0, \rho$ be as in Proposition 40. Then we have

$$
J_{\varepsilon_{n}, \varepsilon_{n}}-J_{\rho, \varepsilon_{n}}=\pi d b^{2} \ln \frac{\rho}{\varepsilon_{n}}+o_{n}(1) .
$$

Proof of Corollary 41. Using Proposition 9, we may consider $\mathbf{x}_{n}=\left(x_{1}^{n}, \ldots, x_{d}^{n}\right) \in \Omega^{d}$ a minimal configuration of points for $J_{\rho, \varepsilon_{n}}$, i.e. s.t.

$$
\hat{\mathcal{J}}_{\rho, \varepsilon_{n}}\left(\mathbf{x}_{n}, \mathbf{1}\right)=J_{\rho, \varepsilon_{n}} .
$$

Combining Corollaries $13 \& 14$ (or Proposition 17 if the pinning term is not periodic), we have the existence of $c>0$ s.t. $B\left(x_{i}^{n}, c \lambda \delta\right) \subset \omega_{\varepsilon}$.

Therefore, for a minimal map $w_{n}$ of $\hat{\mathcal{J}}_{\rho, \varepsilon_{n}}\left(\mathbf{x}_{n}, \mathbf{1}\right)$, we may easily construct a map $\tilde{w}_{n} \in$ $H^{1}\left(\Omega \backslash \cup_{i} \overline{B\left(x_{i}, \varepsilon_{n}\right)}, \mathbb{S}^{1}\right)$ s.t. $\tilde{w}_{n} \in \mathcal{J}_{\varepsilon_{n}}\left(\mathbf{x}_{n}, \mathbf{1}\right)$ and

$$
\begin{aligned}
J_{\varepsilon_{n}, \varepsilon_{n}} & \leq \frac{1}{2} \int_{\Omega \backslash \cup \overline{B\left(x_{i}, \varepsilon_{n}\right)}} U_{\varepsilon_{n}}^{2}\left|\nabla \tilde{w}_{n}\right|^{2} \\
& =\frac{1}{2} \int_{\Omega \backslash \cup \overline{B\left(x_{i}, \rho\right)}} U_{\varepsilon_{n}}^{2}\left|\nabla w_{n}\right|^{2}+\frac{1}{2} \int_{\cup B\left(x_{i}, \rho\right) \backslash \overline{B\left(x_{i}, \varepsilon_{n}\right)}} U_{\varepsilon_{n}}^{2}\left|\nabla \tilde{w}_{n}\right|^{2} \\
& =J_{\rho, \varepsilon_{n}}+d b^{2} \pi \ln \frac{\rho}{\varepsilon_{n}}+o_{n}(1) .
\end{aligned}
$$

On the other hand, Lemma 18 combined with Proposition 40 yield

$$
J_{\rho, \varepsilon_{n}}+d b^{2}\left(\pi \ln \frac{b \rho}{\varepsilon_{n}}+\gamma\right)+o_{n}(1) \leq F_{\varepsilon_{n}}\left(v_{\varepsilon_{n}}\right) \leq J_{\varepsilon_{n}, \varepsilon_{n}}+d b^{2}(\pi \ln b+\gamma) .
$$

We conclude with the help of (4.20) and (4.21). 


\subsubsection{Proof of Theorem 5}

We are now in position to prove Theorem 5, i.e., we are going to prove that

$$
F_{\varepsilon}\left(v_{\varepsilon}\right)=J_{\varepsilon, \varepsilon}+d b^{2}(\pi \ln b+\gamma)+o_{\varepsilon}(1) .
$$

Indeed, using Lemma 18, it suffices to prove that

$$
F_{\varepsilon}\left(v_{\varepsilon}\right) \geq J_{\varepsilon, \varepsilon}+d b^{2}(\pi \ln b+\gamma)+o_{\varepsilon}(1) .
$$

This estimate is equivalent to:

for all $\varepsilon_{n} \downarrow 0$, up to subsequence, we have $F_{\varepsilon_{n}}\left(v_{\varepsilon_{n}}\right) \geq J_{\varepsilon_{n}, \varepsilon_{n}}+d b^{2}(\pi \ln b+\gamma)+o_{n}(1)$.

Let $\varepsilon_{n} \downarrow 0$. Then, up to a subsequence, there is $\rho=\rho_{n}$ given by Proposition 40 s.t.

$$
F_{\varepsilon_{n}}\left(v_{\varepsilon_{n}}\right) \geq J_{\rho, \varepsilon_{n}}+d b^{2}\left(\pi \ln \frac{b \rho}{\varepsilon_{n}}+\gamma\right)+o_{n}(1) .
$$

We deduce from Corollary 41 that

$$
\begin{aligned}
F_{\varepsilon_{n}}\left(v_{\varepsilon_{n}}\right) & \geq J_{\varepsilon_{n}, \varepsilon_{n}}-d b^{2} \ln \frac{\rho}{\varepsilon_{n}}+d b^{2}\left(\pi \ln \frac{b \rho}{\varepsilon_{n}}+\gamma\right)+o_{n}(1) \\
& =J_{\varepsilon_{n}, \varepsilon_{n}}+d b^{2}(\pi \ln b+\gamma)+o_{n}(1)
\end{aligned}
$$

which ends the proof of Theorem 5 .

\subsubsection{Proof of Proposition 40}

In order to construct $\rho$, we first define a suitable extraction.

For $l \in \mathbb{N} \backslash\{0,1\}$, consider $R_{l}$ given by Corollary 39 .

Using Proposition 36 and Corollary [38, for sufficiently large $n, v_{\varepsilon_{n}}$ has exactly $d$ zeros $x_{1}^{n}=x_{1}, \ldots, x_{d}^{n}=x_{d}$.

Clearly, these zeros are well separated and far from $\partial \Omega$ (independently of $n$ ).

Fix $i \in\{1, \ldots, d\}$ and consider

$$
\begin{aligned}
u_{n}^{\prime}: B\left(0, \lambda^{2} \delta^{2} / \varepsilon_{n}\right) & \rightarrow \\
x & \mapsto \frac{u_{\varepsilon_{n}}\left(\frac{\varepsilon_{n}}{b} x+x_{i}\right)}{b} .
\end{aligned}
$$

For simplicity, assume $x_{i}=0$.

Up to a subsequence, one has, as in (4.18),

$$
u_{n}^{\prime} \rightarrow u_{0} \text { in } C_{\mathrm{loc}}^{2}\left(\mathbb{R}^{2}, \mathbb{C}\right), u_{0}(x)=f(|x|) \mathrm{e}^{\imath\left(\theta+\theta_{i}\right)}
$$

where $x=|x| \mathrm{e}^{\imath \theta}, \theta_{i} \in \mathbb{R}$ and $f: \mathbb{R}^{+} \rightarrow \mathbb{R}^{+}$is increasing.

Consequently, for $l \in \mathbb{N} \backslash\{0,1\}$, one may construct an extraction $\left(n_{l}\right)_{l \geq 2}$ s.t., denoting $u_{n_{l}}^{\prime}=u_{l}^{\prime}=\left|u_{l}^{\prime}\right| \mathrm{e}^{\imath\left(\theta+\phi_{l}^{\prime}\right)}$ and $v_{\varepsilon_{n_{l}}}=v_{l}$, we have

$$
\begin{gathered}
\left\{\left|v_{l}\right|<1-1 / l^{2}\right\} \subset \cup_{i} B\left(x_{i}, R_{l} \varepsilon_{n_{l}}\right), \\
\rho_{l}:=R_{l} \varepsilon_{n_{l}} \leq \frac{\lambda^{2} \delta^{2}}{l},
\end{gathered}
$$




$$
\left.\left|\int_{B\left(0, b R_{l}\right)}\right| \nabla u_{l}^{\prime}\right|^{2}+\frac{1}{2}\left(1-\left|u_{l}^{\prime}\right|^{2}\right)^{2}-\int_{B\left(0, b R_{l}\right)}\left|\nabla u_{0}\right|^{2}+\frac{1}{2}\left(1-\left|u_{0}\right|^{2}\right)^{2} \mid \leq \frac{1}{l},
$$

and

$$
\left\|\phi_{l}^{\prime}-\theta_{i}\right\|_{C^{1}\left(\overline{B\left(0, b R_{l}\right)}\right)} \leq \frac{1}{l} .
$$

Here $R_{l} \simeq \sqrt{2} l / b$ and is defined in Corollary 39.

Following the proof of Proposition 1, Step 2 in [7], one has

$$
\int_{B\left(0, \frac{\lambda^{2} \delta^{2}}{\varepsilon_{l}}\right) \backslash \frac{}{B\left(0, R_{l}\right)}}\left|\nabla \phi_{l}^{\prime}\right|^{2} \leq C \text { independently of } l .
$$

In $B\left(0, \lambda^{2} \delta^{2}\right) \backslash \overline{B\left(0, \varepsilon_{n_{l}}\right)}$, we denote $v_{n_{l}}=v_{l}=\left|v_{l}\right| \mathrm{e}^{\imath\left(\theta+\phi_{l}\right)}\left(\mathrm{e}^{\imath \theta}=x /|x|\right)$. By conformal invariance, (4.24) implies that

$$
\left\|\phi_{l}-\theta_{i}\right\|_{L^{\infty}\left(\partial B\left(0, \rho_{l}\right)\right)}+\left|\phi_{l}\right|_{H^{1 / 2}\left(\partial B\left(0, \rho_{l}\right)\right)} \leq \frac{C}{l} .
$$

Denote $W_{l}=B\left(0,2 \rho_{l}\right) \backslash \overline{B\left(0, \rho_{l}\right)}$ and consider $\psi_{i}^{l} \in H^{1 / 2}\left(\partial W_{l}, \mathbb{R}\right)$ s.t.

$$
\psi_{i}^{l}=\left\{\begin{array}{ll}
\phi_{l}-\theta_{i} & \text { on } \partial B\left(0, \rho_{l}\right) \\
0 & \text { on } \partial B\left(0,2 \rho_{l}\right)
\end{array} .\right.
$$

Using (4.26), it is clear that $\left\|\psi_{i}^{l}\right\|_{L^{\infty}\left(\partial W_{l}\right)}+\left|\psi_{i}^{l}\right|_{H^{1 / 2}\left(\partial W_{l}\right)}=\mathcal{O}(1 / l)$. From this, it is straightforward that there exists a constant $C_{0}>0$ (independent of $l$ ) and $\Psi_{i}^{l} \in H^{1}\left(W_{l}, \mathbb{R}\right)$ s.t.

$$
\operatorname{tr}_{\partial W_{l}} \Psi_{i}^{l}=\psi_{i}^{l} \text { and } \frac{1}{2} \int_{W_{l}}\left|\nabla \Psi_{i}^{l}\right|^{2} \leq \frac{C_{0}}{l^{2}}
$$

Finally we define $\Psi_{l} \in H^{1}\left(\Omega \backslash \cup \overline{B\left(x_{i}, \rho_{l}\right)}, \mathbb{R}\right)$ by

$$
\Psi_{l}= \begin{cases}\Psi_{i}^{l}\left(\cdot-x_{i}\right) & \text { in } x_{i}+W_{l} \\ 0 & \text { otherwise }\end{cases}
$$

and

$$
\tilde{w}_{l}=\frac{v_{l}}{\left|v_{l}\right|} \mathrm{e}^{-\imath \Psi_{l}} \in \mathcal{J}_{\rho_{l}}(\mathbf{x}, \mathbf{1}) \text { with } \mathbf{x}=\left(x_{1}, \ldots, x_{d}\right)
$$

Therefore, denoting $w_{l}=\frac{v_{l}}{\left|v_{l}\right|}=\mathrm{e}^{\imath\left(\theta+\phi_{l}\right)}, U_{l}=U_{\varepsilon_{n_{l}}}$ and $\Omega_{\rho_{l}}=\Omega \backslash \overline{B\left(x_{i}, \rho_{l}\right)}$, we have

$$
\hat{\mathcal{J}}_{\rho_{l}, \varepsilon_{n_{l}}}(\mathbf{x}, \mathbf{1}) \leq \frac{1}{2} \int_{\Omega_{\rho_{l}}} U_{l}^{2}\left|\nabla \tilde{w}_{l}\right|^{2}=\frac{1}{2} \int_{\Omega_{\rho_{l}}} U_{l}^{2}\left|\nabla w_{l}\right|^{2}+2 U_{l}^{2} \nabla\left(\theta+\phi_{l}\right) \cdot \nabla \Psi_{l}+o_{l}(1) .
$$

From (4.25), we obtain easily that

$$
\left|\int_{\Omega_{\rho_{l}}} \nabla\left(\theta+\phi_{l}\right) \cdot \nabla \Psi_{l}\right|=\sum_{i}\left|\int_{x_{i}+W_{l}} \nabla\left(\theta+\phi_{l}\right) \cdot \nabla \Psi_{i}^{l}\left(\cdot-x_{i}\right)\right|=o_{l}(1)
$$

and consequently

$$
\hat{\mathcal{J}}_{\rho_{l}, \varepsilon_{n_{l}}}(\mathbf{x}, \mathbf{1}) \leq \frac{1}{2} \int_{\Omega_{\rho_{l}}} U_{l}^{2}\left|\nabla w_{l}\right|^{2}+o_{l}(1) .
$$


On the other hand, from direct computations, one has

$$
\frac{1}{2} \int_{\Omega_{\rho_{l}}} U_{l}^{2}\left|\nabla v_{l}\right|^{2} \geq \frac{1}{2} \int_{\Omega_{\rho_{l}}} U_{l}^{2}\left|\nabla w_{l}\right|^{2}+\frac{1}{2} \int_{\Omega_{\rho_{l}}} U_{l}^{2}\left(\left|v_{l}\right|^{2}-1\right)\left|\nabla\left(\theta+\phi_{l}\right)\right|^{2} .
$$

Using the same argument as Mironescu in [19], one may obtain that

$$
\frac{1}{2} \int_{\Omega_{\rho_{l}}}\left(1-\left|v_{l}\right|^{2}\right)^{1 / 2}\left|\nabla\left(\theta+\phi_{l}\right)\right|^{2} \leq C \text { with } C \text { independent of } l .
$$

From (4.28) and (4.22), we obtain

$$
\frac{1}{2} \int_{\Omega_{\rho_{l}}} U_{l}^{2}\left|\nabla v_{l}\right|^{2} \geq \frac{1}{2} \int_{\Omega_{\rho_{l}}} U_{l}^{2}\left|\nabla w_{l}\right|^{2}-o_{l}(1) .
$$

Therefore, with (4.27),

$$
F_{\varepsilon_{n_{l}}}\left(v_{l}, \Omega_{\rho_{l}}\right)+o_{l}(1) \geq \frac{1}{2} \int_{\Omega_{\rho_{l}}} U_{l}^{2}\left|\nabla v_{l}\right|^{2}+o_{l}(1) \geq \hat{\mathcal{J}}_{\rho_{l}, \varepsilon_{n_{l}}}(\mathbf{x}, \mathbf{1}) .
$$

In order to complete the proof of (4.19), it suffices to estimate the contribution of the discs $B\left(x_{i}, \rho_{l}\right)$.

One has (using (4.23)

$$
\begin{aligned}
F_{\varepsilon_{n_{l}}}\left(v_{l}, B\left(x_{i}, \rho_{l}\right)\right) & =\frac{b^{2}}{2} \int_{B\left(0, \rho_{l}\right)}\left|\nabla\left(\frac{u_{l}}{b}\right)\right|^{2}+\frac{b^{2}}{2 \varepsilon_{n_{l}}^{2}}\left(1-\left|\frac{u_{l}}{b}\right|^{2}\right)^{2}+o_{l}(1) \\
& =\frac{b^{2}}{2} \int_{B\left(0, b R_{l}\right)}\left|\nabla u_{l}^{\prime}\right|^{2}+\frac{1}{2}\left(1-\left|u_{l}^{\prime}\right|^{2}\right)^{2}+o_{l}(1) \\
& =\frac{b^{2}}{2} \int_{B\left(0, b R_{l}\right)}\left|\nabla u_{0}\right|^{2}+\frac{1}{2}\left(1-\left|u_{0}\right|^{2}\right)^{2}+o_{l}(1) .
\end{aligned}
$$

From Proposition 3.11 in [20], one has

$$
\frac{1}{2} \int_{B\left(0, b R_{l}\right)}\left|\nabla u_{0}\right|^{2}+\frac{1}{2}\left(1-\left|u_{0}\right|^{2}\right)^{2}=\pi \ln \left(b R_{l}\right)+\gamma+o_{l}(1),
$$

hence

$$
F_{\varepsilon_{n_{l}}}\left(v_{l}, B\left(x_{i}, \rho_{l}\right)\right)=b^{2}\left[\pi \ln \left(b R_{l}\right)+\gamma\right]+o_{l}(1) .
$$

By combining (4.29) with (4.30), we obtain (4.19) with $\rho_{l}=R_{l} \varepsilon_{n_{l}}$.

\subsection{Proof of Theorems 1, 2, 3 and 4}

We prove Quantization part of Theorem 1 and Theorem 2

- The existence of exactly $d$ zeros is a direct consequence of Corollary 38 .

- The facts that they are well included in $\omega_{\varepsilon}$, well separated and that $v_{\varepsilon}$ has a degree equal to 1 on small circles around the zeros are obtained by Proposition 36 and Corollary 38.

- The lower bound for $\left|v_{\varepsilon}\right|$ is given by Proposition [26, 4 .

We prove Macroscopic location part of Theorem 1 
- The macroscopic location part of Theorem 1 is a direct consequence of Theorem 5 (proved Subsection 4.5.2), Proposition [26, (4.30) and Proposition [16.

Indeed, from Theorem [5, Proposition 26, 4 and (4.30), we get that the zeros form a quasiminimizer of $J_{\rho, \varepsilon}$ (defined Notation 15, page 17). By using Proposition 16 we deduce that they are a quasi-minimizer of the renormalized energy $W_{g}$ (defined Notation 15). Thus, by smoothness of $W_{g}$, the zeros tend to a minimal configuration of $W_{g}$.

We prove Microscopic location part of Theorem 11 \& 4 :

- In the case where $\lambda \rightarrow 0$, the fact that we may localize the zeros inside the inclusions (microscopic location part of Theorem 1 and Theorem 44) is obtained via Theorem 4 in [13.

Indeed we take $f_{n}(x)=\operatorname{tr}_{\partial B\left(\left(k_{n}, l_{n}\right), \delta / 2\right)} v_{\varepsilon_{n}}\left(\left(k_{n}, l_{n}\right)+\delta x\right)$ with $\left(k_{n}, l_{n}\right)$ a center of a cell containing a zero of $v_{\varepsilon_{n}}$. Using the main result of [18], one may easily prove that $f_{n}$ satisfies the conditions (A1) and (A2) in [13]. Thus we can apply Theorem 4 in [13] and infer that the location of the zero inside the inclusion is governed by a renormalized energy which is independent of the boundary condition.

Theorem 3 is obtained by combining:

- The weak $H^{1}$-convergence of $v_{\varepsilon_{n}}$ to $v_{*}$ is a direct consequence of Proposition 31. The limiting equation for $v_{*}$ is a direct consequence of Proposition 32 (this is explained right after Proposition 32).

- The behavior in an $\varepsilon$-neighborhood of the zeros of $v_{\varepsilon_{n}}$ is given by (4.16), (4.17) and (4.18) (noting that in (4.17) we have $R=R_{l} \rightarrow+\infty$ as $l \rightarrow \infty$ ).

\section{A Proof of Proposition 8}

We prove the existence of minimal map in $\mathcal{I}_{\rho}$ and in $\mathcal{J}_{\rho}$. The main ingredient is the fact that these sets are closed under $H^{1}$-weak convergence (see [16] or below). Thus, considering a minimizing sequence for $\frac{1}{2} \int_{\Omega_{\rho}} \alpha|\nabla \cdot|^{2}$ in above sets, we obtained the result.

We consider

- $\theta_{i}: \Omega_{\rho} \rightarrow \mathbb{R}$ the main argument of $x-x_{i}$, i.e. $\mathrm{e}^{\imath \theta_{i}}=\frac{x-x_{i}}{\left|x-x_{i}\right|}$. Note that the $\theta_{i}$ are multivalued function with smooth gradient.

- For $d_{i} \in \mathbb{N}^{*}$ (given by the definition of $\mathcal{I}_{\rho}$ or $\mathcal{J}_{\rho}$ ) we let $\theta_{0}=\sum d_{i} \theta_{i}$ and thus $\mathrm{e}^{\imath \theta_{0}}=$ $\Pi_{i}\left(\frac{x-x_{i}}{\left|x-x_{i}\right|}\right)^{d_{i}}$.

From Lemma 11 in [6], there is $\phi_{0} \in C^{\infty}(\partial \Omega, \mathbb{R})$ s.t. $g \mathrm{e}^{-\imath \theta_{0}}=\mathrm{e}^{\imath \phi_{0}}$.

Note that

$$
\begin{gathered}
w \in \mathcal{I}_{\rho} \Longleftrightarrow w=\mathrm{e}^{\imath\left(\theta_{0}+\phi\right)} \text { with } \phi \in H^{1}\left(\Omega_{\rho}, \mathbb{R}\right) \text { and } \operatorname{tr}_{\partial \Omega} \phi=\phi_{0}, \\
w \in \mathcal{J}_{\rho} \Longleftrightarrow\left\{\begin{array}{c}
w=\mathrm{e}^{\imath\left(\theta_{0}+\phi\right)} \text { with } \phi \in H^{1}\left(\Omega_{\rho}, \mathbb{R}\right), \\
\sum_{j \neq i} d_{j} \theta_{j}+\phi=\mathrm{Cst}_{i} \text { on } \partial B\left(x_{i}, \rho\right) \text { and } \operatorname{tr}_{\partial \Omega} \phi=\phi_{0}
\end{array} .\right.
\end{gathered}
$$

Clearly, from (A.1) and (A.2), $\mathcal{I}_{\rho}$ and $\mathcal{J}_{\rho}$ are $H^{1}$-weakly closed. 
We now prove the second part of Proposition 8 .

One may easily obtain that for some $\lambda: \Omega_{\rho} \rightarrow \mathbb{R}$, denoting $w=\mathrm{e}^{\imath\left(\theta_{0}+\phi\right)}, \phi \in H^{1}\left(\Omega_{\rho}, \mathbb{R}\right)$ (and thus $w \in \mathcal{I}_{\rho}$ ), we have

$$
-\operatorname{div}(\alpha \nabla w)=\lambda w \Longleftrightarrow\left\{-\operatorname{div}\left[\alpha \nabla\left(\theta_{0}+\phi\right)\right]=0 \text { and } \lambda=\alpha|\nabla w|^{2}\right\} .
$$

This observation is a direct consequence of the following identity

$$
-\operatorname{div}\left[\alpha \nabla \mathrm{e}^{\imath\left(\theta_{0}+\phi\right)}\right]=-\operatorname{div}\left[\alpha \nabla\left(\theta_{0}+\phi\right)\right] \imath \mathrm{e}^{\imath\left(\theta_{0}+\phi\right)}+\alpha\left|\nabla\left(\theta_{0}+\phi\right)\right|^{2} \mathrm{e}^{\imath\left(\theta_{0}+\phi\right)} .
$$

Note that under these notations one has $|\nabla w|=\left|\nabla\left(\theta_{0}+\phi\right)\right|$. Thus $w$ is a minimizer in $\mathcal{I}_{\rho}$ or $\mathcal{J}_{\rho}$ if and only if $\theta_{0}+\phi$ minimizes the weighted Dirichlet functional under the condition fixed by the RHS of (A.1) or (A.2).

Consequently, we find that $\theta_{0}+\phi$ minimizes the weighted Dirichlet functional under its Dirichlet boundary condition.

Therefore, we obtain easily that $-\operatorname{div}\left[\alpha \nabla\left(\theta_{0}+\phi\right)\right]=0$. The identity $\nabla\left(\theta_{0}+\phi\right)=$ $w \times \nabla w$ yields $-\operatorname{div}(\alpha \nabla w)=\lambda w$.

Hence, the Euler-Lagrange equations in (3.1) and (3.2) are direct consequences of (A.3).

The condition on the boundary of the holes for $w_{\rho, \alpha}^{\mathrm{deg}}\left(\right.$ resp. $w_{\rho, \alpha}^{\mathrm{Dir}}$ ) follows from multiplying the equation satisfied by $\theta_{0}+\phi_{\rho, \alpha}^{\mathrm{deg}}, w_{\rho, \alpha}^{\mathrm{deg}}=\mathrm{e}^{\imath\left(\theta_{0}+\phi_{\rho, \alpha}^{\mathrm{deg}}\right)}\left(\operatorname{resp} . \theta_{0}+\phi_{\rho, \alpha}^{\mathrm{Dir}}, w_{\rho, \alpha}^{\mathrm{Dir}}=\mathrm{e}^{\imath\left(\theta_{0}+\phi_{\rho, \alpha}^{\mathrm{Dir}}\right)}\right)$ by $\psi \in \mathcal{D}(\Omega, \mathbb{R})\left(\right.$ resp. $\psi \in \mathcal{D}(\Omega, \mathbb{R})$ s.t $\psi \equiv$ Cst $_{i}$ in $\left.B\left(x_{i}, \rho\right)\right)$.

Since $\alpha$ is sufficiently smooth, we can rewrite the Euler-Lagrange equation as

$$
-\Delta \phi=\frac{\nabla \alpha \cdot \nabla\left(\phi+\theta_{0}\right)}{\alpha} \text { with } \frac{\nabla \alpha \cdot \nabla\left(\phi+\theta_{0}\right)}{\alpha} \in L^{2}\left(\Omega_{\rho}\right) .
$$

So, by elliptic regularity $\phi_{\rho, \alpha}^{\mathrm{deg}}, \phi_{\rho, \alpha}^{\mathrm{Dir}} \in H^{2}\left(\Omega_{\rho}, \mathbb{R}\right)$, and consequently $w_{\rho, \alpha}^{\mathrm{deg}}, w_{\rho, \alpha}^{\mathrm{Dir}} \in H^{2}\left(\Omega_{\rho}, \mathbb{S}^{1}\right)$.

\section{B Proof of Proposition 9}

We prove the existence of a minimal configuration $\{\mathbf{x}, \mathbf{d}\}=\left\{\left(x_{1}, \ldots, x_{N}\right),\left(d_{1}, \ldots, d_{n}\right)\right\}$ for $I_{\rho, \alpha}$.

Let $\left(\left\{\mathbf{x}_{n}, \mathbf{d}_{n}\right\}\right)_{n}$ be a minimizing sequence of configuration of $I_{\rho, \alpha}$, i.e.,

$$
\inf _{\substack{w \in H^{1}\left(\Omega_{\rho}^{n}, \mathbb{S}^{1}\right) \text { s.t. } \\ w=g \text { in } \Omega^{\prime} \backslash \Omega \bigcup \cup\left(x_{i}^{n}, \rho\right)}} \frac{1}{2} \int_{\Omega_{\rho}^{n}} \alpha|\nabla w|^{2} \rightarrow I_{\rho, \alpha} ;
$$

here $\Omega_{\rho}^{n}=\Omega^{\prime} \backslash \cup \overline{B\left(x_{i}^{n}, \rho\right)}$.

Up to a subsequence, we have $N_{n}=N=$ Cst, $\mathbf{d}_{n}=\mathbf{d}=$ Cst and $\mathbf{x}_{n} \rightarrow \mathbf{x}$ with $\mathbf{x}=\left(x_{1}, \ldots, x_{N}\right)$ s.t. $\min _{i \neq j}\left|x_{i}-x_{j}\right| \geq 8 \rho$.

Consider $w_{n} \in \mathcal{I}_{\rho}\left(\mathbf{x}_{n}, \mathbf{d}\right)$ a minimal map. Since $w_{n}$ is bounded independently of $n$ in $H^{1}\left(\Omega_{\rho}^{n}\right)$, up to a subsequence, we have $w_{n} \rightarrow w_{0}$ in $H_{\mathrm{loc}}^{1}\left(\Omega_{\rho}^{0}\right), \Omega_{\rho}^{0}=\Omega^{\prime} \backslash \cup \overline{B\left(x_{i}, \rho\right)}$.

Clearly the following properties hold:

- $w_{0} \in H_{\mathrm{loc}}^{1}\left(\Omega_{\rho}^{0}, \mathbb{S}^{1}\right)$ and $w_{0}=g$ in $\Omega_{\rho}^{0} \backslash \bar{\Omega}$.

- For all compact $K \subset \Omega_{\rho}^{0}$ we have $\frac{1}{2} \int_{K} \alpha\left|\nabla w_{0}\right|^{2} \leq \liminf \frac{1}{2} \int_{K} \alpha\left|\nabla w_{n}\right|^{2} \leq I_{\rho, \alpha}$. 
Thus $w_{0} \in H_{g}^{1}\left(\Omega_{\rho}^{0}, \mathbb{S}^{1}\right)$ and $\int_{\Omega_{\rho}^{0}} \alpha\left|\nabla w_{0}\right|^{2} \leq I_{\rho, \alpha}$.

Now, it suffices to check that $\operatorname{deg}_{\partial B\left(x_{i}, \rho\right)}\left(w_{0}\right) \in \mathbb{N}^{*}$ for all $i$. Since $w_{0}$ is $\mathbb{S}^{1}$-valued, this fact is equivalent to $\operatorname{deg}_{\partial B\left(x_{i}, \rho^{\prime}\right)}\left(w_{0}\right) \in \mathbb{N}^{*}$ for all $i$ and for all $\rho^{\prime} \in(\rho, 2 \rho)$.

In view of the facts:

- for $\rho^{\prime} \in(\rho, 2 \rho)$ we have $w_{n}^{\prime}=w_{n \mid \Omega^{\prime} \backslash \cup \overline{B\left(x_{i}, \rho^{\prime}\right)}} \rightarrow w_{0}^{\prime}=w_{0 \mid \Omega^{\prime} \backslash \cup \overline{B\left(x_{i}^{n}, \rho^{\prime}\right)}}$

- the set $\mathcal{I}^{\prime}:=\left\{w^{\prime} \in H^{1}\left(\Omega^{\prime} \backslash \cup \overline{B\left(x_{i}, \rho^{\prime}\right)}, \mathbb{S}^{1}\right) \mid \operatorname{deg}_{\partial B\left(x_{i}, \rho^{\prime}\right)}\left(w^{\prime}\right)=d_{i}\right.$ for all $\left.i \in\{1, \ldots, N\}\right\}$ is closed under the $H^{1}$-weak convergence (see Appendix $\mathrm{A}$ or [16]),

since $w_{n}^{\prime} \in \mathcal{I}^{\prime}$, we obtain that $w_{0}^{\prime} \in \mathcal{I}^{\prime}$. Therefore $\{\mathbf{x}, \mathbf{d}\}=\left\{\left(x_{1}, \ldots, x_{N}\right),\left(d_{1}, \ldots, d_{n}\right)\right\}$ is a minimal configuration for $I_{\rho, \alpha}$.

Now we prove the existence of a minimal configuration for $J_{\rho, \alpha}$.

Let $\left(\mathbf{x}_{n}\right)_{n}$ be a minimizing sequence of configuration for $J_{\rho, \alpha}$, i.e.,

$$
\hat{\mathcal{J}}_{\rho, \alpha}\left(\mathbf{x}_{n}, \mathbf{1}\right) \rightarrow J_{\rho, \alpha}
$$

Up to a subsequence, one may assume that there is $\mathbf{x}=\left(x_{1}, \ldots, x_{d}\right) \in \Omega^{d}$ s.t. $x_{i}^{n} \rightarrow x_{i}$, $\left|x_{i}-x_{j}\right| \geq 8 \rho$ and $\operatorname{dist}\left(x_{i}, \partial \Omega\right) \geq 8 \rho$.

Let $\eta_{n}=8 \max \left|x_{i}^{n}-x_{i}\right|$. There is a smooth diffeomorphism $\phi_{n}: \mathbb{R}^{2} \rightarrow \mathbb{R}^{2}$ satisfying

$$
\left\{\begin{array}{ll}
\phi_{n} \equiv \operatorname{Id}_{\mathbb{R}^{2}} & \text { in } \mathbb{R}^{2} \backslash \cup \overline{B\left(x_{i}^{n}, \rho+\eta_{n}^{1 / 2}\right)} \\
\phi_{n}\left[x_{i}+\left(1+\eta_{n}\right) x\right]=x_{i}^{n}+x & \text { for } x \in B(0, \rho) \\
\left\|\phi_{n}-\operatorname{Id}_{\mathbb{R}^{2}}\right\|_{C^{1}\left(\mathbb{R}^{2}\right)}=o_{n}(1) &
\end{array} .\right.
$$

For example we can consider $\phi_{n}=\operatorname{Id}_{\mathbb{R}^{2}}+H_{n}$ with

$$
\left\{\begin{array}{ll}
H_{n} \equiv 0 & \text { in } \mathbb{R}^{2} \backslash \overline{\cup B\left(x_{i}^{n}, \rho+\eta_{n}^{1 / 2}\right)} \\
H_{n}\left[x_{i}+\left(1+\eta_{n}\right) x\right]=\left[1-\psi_{n}(|x|)\right]\left(x_{i}^{n}-x_{i}-\eta_{n} x\right) & \text { for } x \in B\left(0, \frac{\rho+\eta_{n}^{1 / 2}}{1+\eta_{n}}\right)
\end{array} .\right.
$$

Here $\psi_{n}: \mathbb{R}^{+} \rightarrow[0,1]$ is a smooth function satisfying

$$
\psi_{n}(r)=\left\{\begin{array}{ll}
0 & \text { if } r \leq \rho \\
1 & \text { if } r \geq \rho+\eta_{n}^{1 / 2} / 2
\end{array} \text { and }\left|\psi_{n}^{\prime}\right|=\mathcal{O}\left(\eta_{n}^{-1 / 2}\right) .\right.
$$

For $w_{n} \in \mathcal{J}_{\rho}\left(\mathbf{x}_{n}, \mathbf{1}\right)$ a minimal map, we consider

$$
\begin{array}{ccc}
\tilde{w}_{n}: \Omega \backslash \cup_{i} \overline{B\left(x_{i},\left(1+\eta_{n}\right) \rho\right)} & \rightarrow & \mathbb{S}^{1} \\
x & \mapsto w_{n}\left[\phi_{n}(x)\right]
\end{array}
$$

Clearly $\tilde{w}_{n}$ is well defined and we have

$$
\begin{aligned}
& \int_{\Omega \backslash \cup_{i} \overline{B\left(x_{i},\left(1+\eta_{n}\right) \rho\right)}} \alpha\left|\nabla \tilde{w}_{n}\right|^{2}=\int_{\Omega \backslash \cup_{i} \overline{B\left(x_{i}^{n}, \rho\right)}} \alpha\left|\nabla w_{n}\right|^{2}+o_{n}(1), \\
& \tilde{w}_{n}\left[x_{i}+\left(1+\eta_{n}\right) \rho \mathrm{e}^{\imath \theta}\right]=w_{n}\left[\phi\left(x_{i}+\left(1+\eta_{n}\right) \rho \mathrm{e}^{\imath \theta}\right)\right]=w_{n}\left[x_{i}^{n}+\rho \mathrm{e}^{\imath \theta}\right]=\mathrm{e}^{\imath\left(\theta+\theta_{i}\right)}
\end{aligned}
$$


We can extend $\tilde{w}_{n}$ in $\cup_{i} B\left(x_{i},\left(1+\eta_{n}\right) \rho\right) \backslash \overline{B\left(x_{i}, \rho\right)}$ by $\tilde{w}_{n}\left(x_{i}+r \mathrm{e}^{\imath \theta}\right)=\mathrm{e}^{\imath\left(\theta+\theta_{i}\right)}, \rho<r<$ $\left(1+\eta_{n}\right) \rho$.

Clearly, we have $\tilde{w}_{n} \in \mathcal{J}_{\rho, \alpha}(\mathbf{x}, \mathbf{1})$ and $\frac{1}{2} \int_{\Omega \backslash \cup_{i} \overline{B\left(x_{i}, \rho\right)}} \alpha\left|\nabla \tilde{w}_{n}\right|^{2}=J_{\rho, \alpha}+o_{n}(1)$.

Thus considering $w \in \mathcal{J}_{\rho, \alpha}(\mathbf{x}, \mathbf{1})$ a minimizer of $\frac{1}{2} \int_{\Omega \backslash \cup_{i} \overline{B\left(x_{i}, \rho\right)}} \alpha|\nabla \cdot|^{2}$, we obtain

$$
\frac{1}{2} \int_{\Omega \backslash \cup_{i} \overline{B\left(x_{i}, \rho\right)}} \alpha|\nabla w|^{2} \leq \frac{1}{2} \int_{\Omega \backslash \cup_{i} \overline{B\left(x_{i}, \rho\right)}} \alpha\left|\nabla \tilde{w}_{n}\right|^{2}=J_{\rho, \alpha}+o_{n}(1) .
$$

Letting $n \rightarrow \infty$ we deduce that the configuration $\mathbf{x}=\left(x_{1}, \ldots, x_{d}\right)$ is minimal.

\section{Proof of Proposition 10}

As explained Section 3.2. Proposition 10 is easily established when either $N=1$ or when the points are well separated. It remains to consider the case where $N \geq 2$ and there are $i \neq j$ s.t. $\left|x_{i}-x_{j}\right| \leq 4 \eta_{\text {stop }}$.

\section{C.1 The separation process}

We assume that $N \geq 2$ and that the points are not well separated. Our purpose is to compare the energy of $\overline{\mathcal{J}}_{\rho, \alpha}$ to the energy of $\hat{\mathcal{I}}_{\rho, \alpha}$. To this purpose, we decompose $\Omega_{\rho}$ into several regions and we compare energies in each regions. These regions are constructed recursively using the following version of Theorem IV.1 in [4.

Lemma 42. Let $N \geq 2, x_{1}, \ldots, x_{N} \in \mathbb{R}^{2}$ and $\eta>0$. There are $\kappa \in\left\{9^{0}, \ldots, 9^{N-1}\right\}$ and $\left\{y_{1}, \ldots, y_{N^{\prime}}\right\} \subset\left\{x_{1}, \ldots, x_{N}\right\}$ s.t.

$$
\cup_{i=1}^{N} B\left(x_{i}, \eta\right) \subset \cup_{i=1}^{N^{\prime}} B\left(y_{i}, \kappa \eta\right)
$$

and

$$
\left|y_{i}-y_{j}\right| \geq 8 \kappa \eta \text { for } i \neq j \text {. }
$$

We let $x_{1}^{0}, \ldots, x_{N}^{0}$ denote the initial points $x_{1}, \ldots, x_{N}$ and $N_{0}=N$ the initial number of points. For $k \geq 1$ (here, $k$ is an iteration in the construction of the regions), we let $N_{k}$ denote the number of points selected at Step $k$, and denote the points we select by $x_{1}^{k}, \ldots, x_{N_{k}}^{k}$.

The recursive construction is made in such a way that $N_{k}>N_{k+1}$ and $N_{k} \geq 1$ for all $k \geq 1$.

The process will stop at the end of Step $k$ if and only if one of the following conditions yields

Rule 1: there is a unique point in the selection (i.e. $N_{k}=1$ ),

Rule 2: $\min _{i \neq j}\left|x_{i}^{k}-x_{j}^{k}\right|>4 \eta_{\text {stop }}$.

Step $k, k \geq 1$ : Let $\eta_{k}^{\prime}=\frac{1}{4} \min _{i \neq j}\left|x_{i}^{k-1}-x_{j}^{k-1}\right|$.

Using Lemma 42, there are

$$
\kappa_{k} \in\left\{9^{1}, \ldots, 9^{N_{k-1}-1}\right\} \text { and }\left\{x_{1}^{k}, \ldots, x_{N_{k}}^{k}\right\} \subset\left\{x_{1}^{k-1}, \ldots, x_{N_{k-1}}^{k-1}\right\}
$$


s.t.

$$
\cup_{i=1}^{N_{k-1}} B\left(x_{i}^{k-1}, \eta_{k}^{\prime}\right) \subset \cup_{i=1}^{N_{k}} B\left(x_{i}^{k}, \kappa_{k} \eta_{k}^{\prime}\right) \text { and }\left|x_{i}^{k}-x_{j}^{k}\right| \geq 8 \kappa_{k} \eta_{k}^{\prime} \text { for } i \neq j .
$$

We denote $\eta_{k}=2 \kappa_{k} \eta_{k}^{\prime}$. We stop the construction if $N_{k}=1$ (Rule 1) or if $\frac{1}{4} \min \mid x_{i}^{k-1}$ $x_{j}^{k-1} \mid>\eta_{\text {stop }}($ Rule 2$)$.

In Figure 4 \& 5 both stop-conditions are presented.

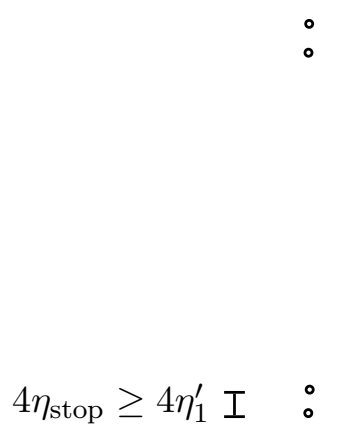

(a) The initial balls
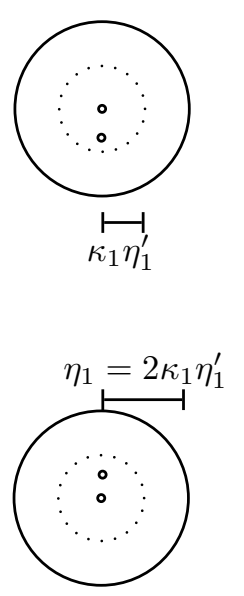

(b) The first step: a selection of two centers

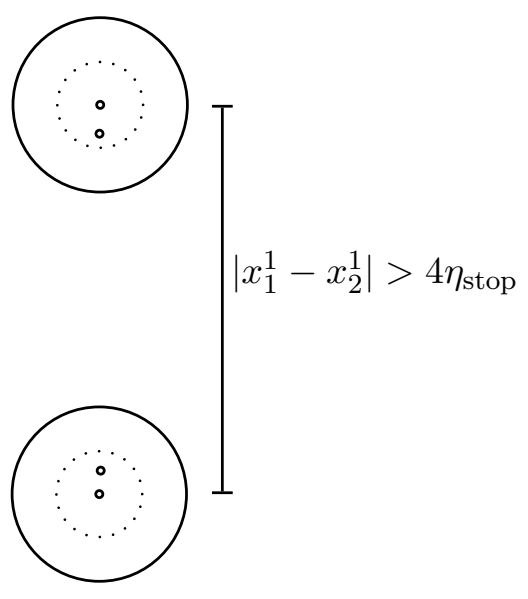

(c) The process stops at the end of the first step since there are two well separated balls.

Figure 4: The process stops when we obtain well separated balls

$\because$

$\therefore$
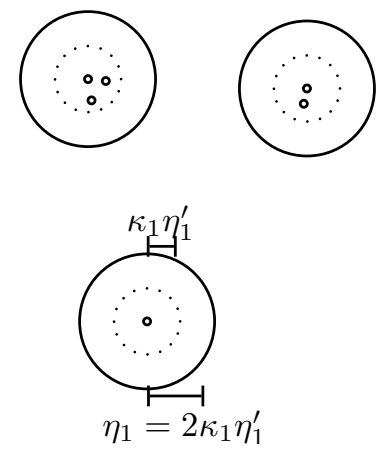

(b) The first step: a selection of three centers

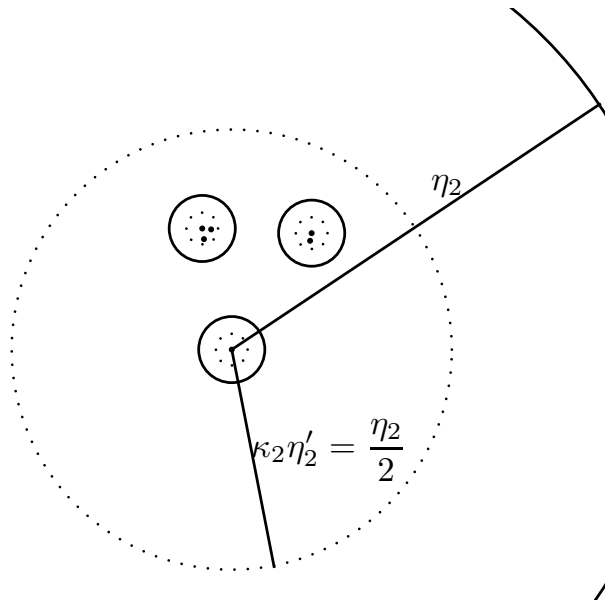

(c) The second step: it remains a unique ball (the picture is at scale $1 / 2$ )

Figure 5: The process stops when we obtain a unique ball

Remark 43. i. From the definitions of $\eta_{k}^{\prime}$ and $\eta_{k}$, we have $N_{k}<N_{k-1}$ and $\eta_{k-1} \leq \eta_{k}^{\prime}<$ $\eta_{k}$

ii. The balls $B\left(x_{j}^{k}, 2 \eta_{k}\right)$ are disjoint.

iii. Denoting $\Lambda_{j}^{k} \subset\left\{1, \ldots, N_{k-1}\right\}$ the set of indices $i$ s.t. $x_{i}^{k-1} \in B\left(x_{j}^{k}, \kappa_{k} \eta_{k}^{\prime}\right)$, then for $i \in \Lambda_{j}^{k}$ 
we have $B\left(x_{i}^{k-1}, \eta_{k}^{\prime}\right) \subset B\left(x_{j}^{k}, \kappa_{k} \eta_{k}^{\prime}\right)$. Furthermore, by construction, $\left|x_{i}^{k-1}-x_{j}^{k-1}\right| \geq$ $4 \eta_{k}^{\prime}$.

\section{C.2 The separation process gives a natural partition of $\Omega$}

Let $\Omega, g, x_{1}, \ldots, x_{N}, \mathbf{d}$ and $\rho, \eta_{\text {stop }}$ like in Section 3.2 with $N \geq 2$ and s.t. the points are not well separated.

We apply the separation process. The process stops after $K$ steps, $1 \leq K \leq N-1$.

We denote

$\left\{y_{1}, \ldots, y_{N^{\prime}}\right\} \subset\left\{x_{1}, \ldots, x_{N}\right\}$ the selection that we obtain, i.e., $y_{j}=x_{j}^{K}$ and $N^{\prime}=N_{K}$,

$$
\begin{gathered}
\eta=\left\{\begin{array}{ll}
9^{N} \cdot \eta_{\text {stop }} & \text { if } N^{\prime}=1 \\
\min \left\{9^{N} \cdot \eta_{\text {stop }}, \frac{1}{4} \min \left|y_{i}-y_{j}\right|\right\} & \text { if } N^{\prime}>1
\end{array}, \text { so } \eta \geq \max \left(\eta_{K}, \eta_{\text {stop }}\right),\right. \\
\Lambda_{j}=\left\{i \in\{1, \ldots, N\} \mid x_{i} \in B\left(y_{j}, \eta\right)\right\} \text { and } \eta_{0}=\rho .
\end{gathered}
$$

We denote

$$
\begin{gathered}
D_{j, k}=B\left(x_{j}^{k}, \eta_{k}\right) \backslash \cup_{x_{i}^{k-1} \in B\left(x_{j}^{k}, \eta_{k}\right)} \overline{B\left(x_{i}^{k-1}, \eta_{k}^{\prime}\right)}, k \in\{1, \ldots, K\}, j \in\left\{1, \ldots, N_{k}\right\}, \\
R_{j, k}=B\left(x_{j}^{k}, \eta_{k+1}^{\prime}\right) \backslash \overline{B\left(x_{j}^{k}, \eta_{k}\right)}, k \in\{0, \ldots, K-1\}, j \in\left\{1, \ldots, N_{k}\right\}, \\
R_{j}=B\left(y_{j}, \eta\right) \backslash \overline{B\left(y_{j}, \eta_{K}\right)}, j \in\left\{1, \ldots, N^{\prime}\right\}
\end{gathered}
$$

and

$$
D=\Omega \backslash \cup_{j \in\left\{1, \ldots, N^{\prime}\right\}} \overline{B\left(y_{j}, \eta\right)} .
$$

Note that by construction of $\eta_{k}^{\prime}, \eta_{k}$ and $x_{i}^{k}$ the following properties are satisfied:

$$
\text { the balls } B\left(x_{i}^{k-1}, 2 \eta_{k}^{\prime}\right) \text { are disjoint }
$$

and

$$
2 \cdot 9 \eta_{k}^{\prime} \leq \eta_{k} \leq 9^{N} \eta_{k}^{\prime}
$$

Therefore

$$
\Omega_{\rho}=D \bigcup \cup_{j, k} \overline{D_{j, k}} \bigcup \cup_{j, k} R_{j, k} \bigcup \cup_{j} \overline{R_{j}} \text { with disjoint unions. }
$$

\section{C.3 Construction of test functions}

\section{Construction of test functions in $D$ and $D_{j, k}$}

Lemma 44. 1. Let $\eta>0$. There is $C_{1}(\eta)>0$ (depending on $\Omega, g$ and $\eta$ ) s.t. if $x_{1}, \ldots, x_{N} \in \Omega$ satisfy $\min _{i \neq j}\left|x_{i}-x_{j}\right|, \min _{i} \operatorname{dist}\left(x_{i}, \partial \Omega\right)>4 \eta$ and $d_{1}, \ldots, d_{N} \in \mathbb{N}^{*}$ are s.t. $\sum d_{i}=d$ then there is $w \in H_{g}^{1}\left(\Omega \backslash \cup \overline{B\left(x_{i}, \eta\right)}, \mathbb{S}^{1}\right)$ s.t. $w(x)=\frac{\left(x-x_{i}\right)^{d_{i}}}{\eta^{d_{i}}}$ on $\partial B\left(x_{i}, \eta\right)$ and

$$
\int_{\Omega \backslash \cup \overline{B\left(x_{i}, \eta\right)}}|\nabla w|^{2} \leq C_{1}(\eta)
$$

Moreover $C_{1}$ can be considered decreasing with $\eta$. 

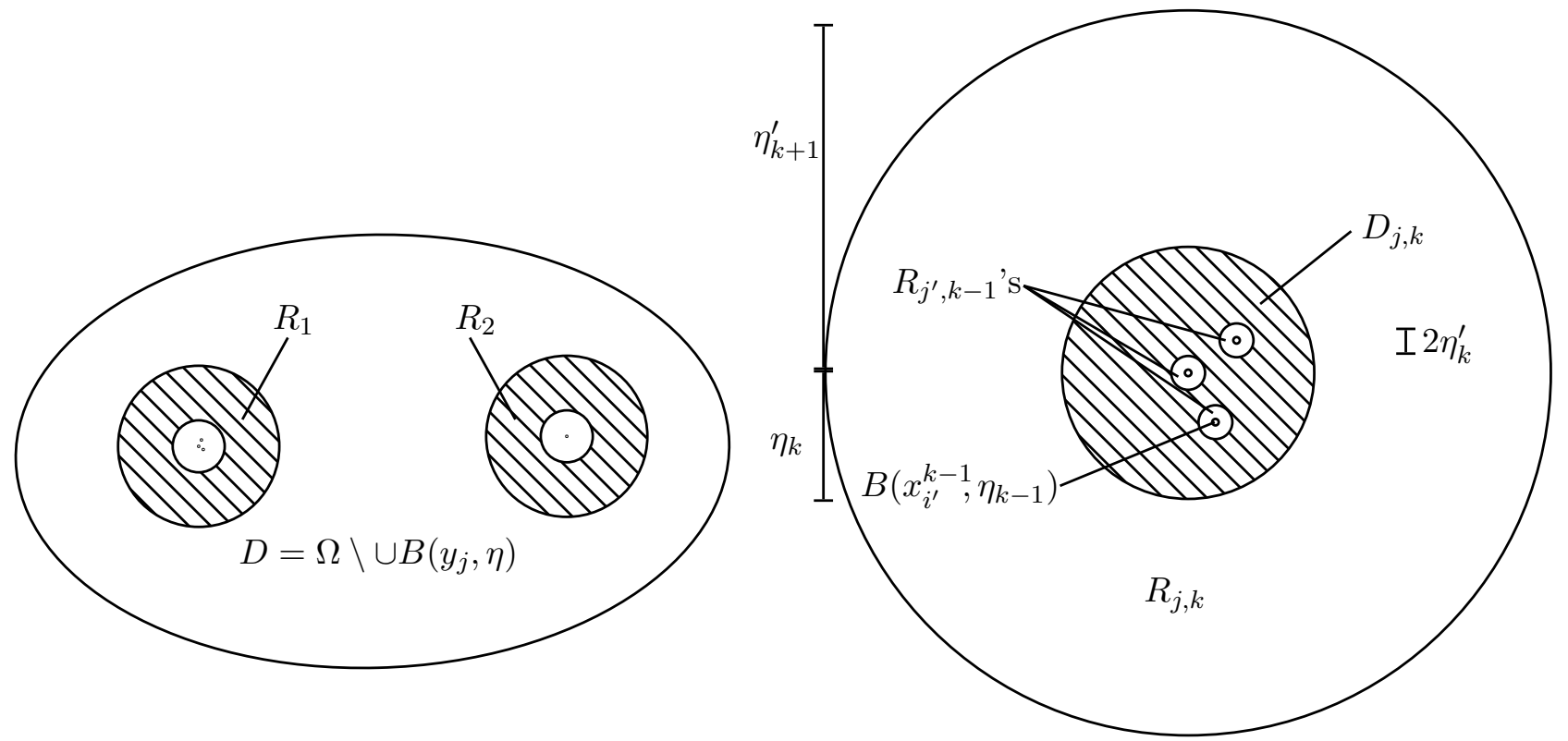

(a) The macroscopic perforated domain and the first mesoscopic rings

(b) A mesoscopic ring and a mesoscopic perforated domain

2. Let $\eta>0, \kappa \geq 8, d_{0}, d_{1}, \ldots, d_{N} \in \mathbb{N}^{*}$ be s.t. $\sum_{1 \leq i \leq N} d_{i}=d_{0}$. Then, there is $C_{2}\left(\kappa, d_{0}\right)$ s.t. for $x_{1}, \ldots, x_{N} \in B(0, \kappa \eta)$ satisfying $\min _{i \neq j}\left|x_{i}-x_{j}\right| \geq 4 \eta$ we can associate a map $w \in H^{1}\left(B(0,2 \kappa \eta) \backslash \cup \overline{B\left(x_{i}, \eta\right)}, \mathbb{S}^{1}\right)$ s.t.

$$
w(x)= \begin{cases}\frac{x^{d_{0}}}{(2 \kappa \eta)^{d_{0}}} & \text { on } \partial B(0,2 \kappa \eta) \\ \frac{\left(x-x_{i}\right)^{d_{i}}}{\eta^{d_{i}}} & \text { on } \partial B\left(x_{i}, \eta\right)\end{cases}
$$

and

$$
\int_{B(0,2 \kappa \eta) \backslash \cup \overline{B\left(x_{i}, \eta\right)}}|\nabla w|^{2} \leq C_{2}\left(\kappa, d_{0}\right) .
$$

Moreover $C_{2}$ can be considered increasing with $\kappa, d_{0}$.

Proof. In order to prove 1., we consider, e.g., the test function defined in $\Omega_{\eta}:=\Omega \backslash \cup \overline{B\left(x_{i}, \eta\right)}$ by

$w=\mathrm{e}^{\imath H} \Pi_{i} \frac{\left(x-x_{i}\right)^{d_{i}}}{\left|x-x_{i}\right|^{d_{i}}}$ with $H$ s.t. $\left\{\begin{array}{l}H: \Omega_{\eta} \rightarrow \mathbb{R} \\ H \equiv 0 \text { in }\left\{\operatorname{dist}\left[x, \partial \Omega_{\eta}\right] \geq \eta\right\} \\ -\Delta H=0 \text { in }\left\{\operatorname{dist}\left[x, \partial \Omega_{\eta}\right]<\eta\right\} \\ w \in H_{g}^{1}\left(\Omega_{\eta}, \mathbb{S}^{1}\right) \text { and } w(x)=\frac{\left(x-x_{i}\right)^{d_{i}}}{\eta^{d_{i}}} \text { on } \partial B\left(x_{i}, \eta\right)\end{array}\right.$

Assertion 2. was essentially established in [14], Section 3. We adapt here the argument 
in [14]. By conformal invariance, we may assume that $\eta=1$. We let

$$
w(x)= \begin{cases}\Pi_{i} \frac{\left[x+2 x_{i}\left(\frac{|x|}{\kappa}-2\right)\right]^{d_{i}}}{\left|x+x_{i}\left(\frac{|x|}{\kappa}-2\right)\right|^{d_{i}}} & \text { in } B(0,2 \kappa) \backslash \overline{B\left(0, \frac{3 \kappa}{2}\right)} \\ \prod_{i} \frac{\left(x-x_{i}\right)^{d_{i}}}{\left|x-x_{i}\right|^{d_{i}}} & \text { in } B\left(0, \frac{3 \kappa}{2}\right) \backslash \cup \overline{B\left(x_{i}, 3 / 2\right)} \\ \frac{\left(x-x_{i}\right)^{d_{i}}}{\left|x-x_{i}\right|^{d_{i}}} \mathrm{e}^{\imath\left(2\left|x-x_{i}\right|-2\right) \varphi_{i}} & \text { in } B\left(x_{i}, 3 / 2\right) \backslash \cup \overline{B\left(x_{i}, 1\right)}\end{cases}
$$

here $\varphi_{i} \in C^{\infty}\left(B\left(x_{i}, 3 / 2\right), \mathbb{R}\right)$ is defined by $\mathrm{e}^{\imath \varphi_{i}}=\Pi_{j \neq i} \frac{\left(x-x_{j}\right)^{d_{j}}}{\left|x-x_{j}\right|^{d_{j}}}$ and $\varphi_{i}\left(x_{i}\right) \in[0,2 \pi)$. Clearly $\left\|\varphi_{i}\right\|_{H^{1}\left(B\left(x_{i}, 3 / 2\right) \backslash \overline{B\left(x_{i}, 1\right)}\right)}$ is bounded by a constant which depends only on $d_{0}$.

By (C.1) and Lemma 44, 1 , one may find a map $w_{0} \in H^{1}\left(D, \mathbb{S}^{1}\right)$ s.t.

$$
w_{0}=\left\{\begin{array}{ll}
g & \text { on } \partial \Omega \\
w_{0}(x)=\frac{\left(x-y_{j}\right)^{\tilde{d}_{j}}}{\eta^{\tilde{d}_{j}}} & \text { on } \partial B\left(y_{j}, \eta\right)
\end{array}\left(\text { where } \tilde{d}_{j}=\sum_{x_{i} \in B\left(y_{j}, \eta\right)} d_{i}\right)\right.
$$

satisfying in addition

$$
\int_{D}\left|\nabla w_{0}\right|^{2} \leq C_{1}(\eta) \leq C_{1}\left(\eta_{\text {stop }}\right)
$$

For each $D_{j, k}$, combining (C.2), (C.5), and using Lemma 44,2, there exists a map $w_{j, k} \in H^{1}\left(D_{j, k}, \mathbb{S}^{1}\right)$ s.t.

$$
w_{j, k}(x)=\left\{\begin{array}{ll}
\frac{\left(x-x_{j}^{k}\right)^{\tilde{d}_{j, k}}}{\eta_{k}} & \text { for } x \in \partial B\left(x_{j}^{k}, \eta_{k}\right) \\
\frac{\left(x-x_{i}^{k-1}\right)^{\tilde{d}_{i, k-1}}}{\eta_{k}^{\tilde{d}_{i, k-1}}} & \text { for } x \in \partial B\left(x_{i}^{k-1}, \eta_{k}^{\prime}\right)
\end{array} .\right.
$$

Here,

$$
\tilde{d}_{j, k}=\sum_{x_{i} \in B\left(x_{j}^{k}, \eta_{k}\right)} d_{i}
$$

and

$$
\int_{D_{j, k}}\left|\nabla w_{j, k}\right|^{2} \leq C_{2}\left(2 \kappa_{k}, d_{j, k}\right) \leq C_{2}\left(2 \cdot 9^{d-1}, d\right)
$$

Construction of test functions in $R_{j}$ 's and $R_{j, k}$ 's

For $R>r>0$ and $x_{0} \in \mathbb{R}^{2}$ we denote $\mathscr{R}\left(x_{0}, R, r\right):=B\left(x_{0}, R\right) \backslash \overline{B\left(x_{0}, r\right)}$. For $\alpha \in$ $L^{\infty}\left(\mathbb{R}^{2},\left[b^{2}, 1\right]\right)$, we define

$$
\mu_{\alpha}\left(\mathscr{R}\left(x_{0}, R, r\right), \tilde{d}\right)=\inf _{\substack{w \in H^{1}\left(\mathscr{R}\left(x_{0}, R, r\right), \mathbb{S}^{1}\right) \\ \operatorname{deg}_{\partial B\left(x_{0}, R\right)}(w)=\tilde{d}}} \frac{1}{2} \int_{\mathscr{R}\left(x_{0}, R, r\right)} \alpha|\nabla w|^{2}
$$


and

$$
\mu_{\alpha}^{\operatorname{Dir}}\left(\mathscr{R}\left(x_{0}, R, r\right), \tilde{d}\right)=\inf _{\begin{array}{c}
w \in H^{1}\left(\mathscr{R}\left(x_{0}, R, r\right), \mathbb{S}^{1}\right) \\
w\left(x_{0}+R \mathrm{e}^{\imath \theta}\right)=\mathrm{e}^{2 \tilde{d} \theta} \\
w\left(x_{0}+r \mathrm{e}^{2 \theta}\right) \mathrm{e}^{-\imath \tilde{d} \theta}=\text { Cst }
\end{array}} \frac{1}{2} \int_{\mathscr{R}\left(x_{0}, R, r\right)} \alpha|\nabla w|^{2} .
$$

In the special case $\alpha=U_{\varepsilon}^{2}$, we denote

$$
\mu_{\varepsilon}\left(\mathscr{R}\left(x_{0}, R, r\right), \tilde{d}\right)=\mu_{U_{\varepsilon}^{2}}\left(\mathscr{R}\left(x_{0}, R, r\right), \tilde{d}\right)
$$

and

$$
\mu_{\varepsilon}^{\operatorname{Dir}}\left(\mathscr{R}\left(x_{0}, R, r\right), \tilde{d}\right)=\mu_{U_{\varepsilon}^{2}}^{\operatorname{Dir}}\left(\mathscr{R}\left(x_{0}, R, r\right), \tilde{d}\right) .
$$

Note that the minimization problems (C.10) and (C.11) admit solutions; this is obtained by adapting the proof of Proposition 8 ,

We present an adaptation of a result of Sauvageot, Theorem 2 in [21.

Proposition 45. There is $C_{b}>0$ depending only on $b \in(0,1)$ s.t. for $R>r>0$ and $\alpha \in L^{\infty}\left(\mathbb{R}^{2}, \mathbb{R}\right)$ satisfying $1 \geq \alpha \geq b^{2}$, we have

$$
\mu_{\alpha}^{\operatorname{Dir}}\left(\mathscr{R}\left(x_{0}, R, r\right), \tilde{d}\right) \leq \mu_{\alpha}\left(\mathscr{R}\left(x_{0}, R, r\right), \tilde{d}\right)+\tilde{d}^{2} C_{b} .
$$

Proof. This result was obtained by Sauvageot with $\alpha \in W^{1, \infty}\left(\mathbb{R}^{2},\left[b^{2}, 1\right]\right)$. We may extend this estimate to $\alpha \in L^{\infty}\left(\mathbb{R}^{2},\left[b^{2}, 1\right]\right)$.

Indeed, let $\left(\rho_{t}\right)_{1>t>0}$ be a classical mollifier, namely $\rho_{t}(x)=t^{-2} \rho(x / t)$ with $\rho \in$ $C^{\infty}\left(\mathbb{R}^{2},[0,1]\right)$, Supp $\rho \subset B(0,1)$ and $\int_{\mathbb{R}^{2}} \rho=1$.

Set $\alpha_{t}=\alpha * \rho_{t} \in W^{1, \infty}\left(B\left(x_{0}, R\right),\left[b^{2}, 1\right]\right)$. We have

$$
\lim _{t \rightarrow 0} \mu_{\alpha_{t}}\left(\mathscr{R}\left(x_{0}, R, r\right), \tilde{d}\right)=\mu_{\alpha}\left(\mathscr{R}\left(x_{0}, R, r\right), \tilde{d}\right)
$$

and

$$
\lim _{t \rightarrow 0} \mu_{\alpha_{t}}^{\operatorname{Dir}}\left(\mathscr{R}\left(x_{0}, R, r\right), \tilde{d}\right)=\mu_{\alpha}^{\operatorname{Dir}}\left(\mathscr{R}\left(x_{0}, R, r\right), \tilde{d}\right) .
$$

We prove (C.12), Equality (C.13) follows with the same lines.

Let $w$ be a minimizer of $\mu_{\alpha}\left(\mathscr{R}\left(x_{0}, R, r\right), \tilde{d}\right)$. By using Dominated convergence theorem, since $\alpha_{t} \rightarrow \alpha$ in $L^{1}\left(B\left(x_{0}, R\right)\right)$, we obtain that $\alpha_{t}|\nabla w|^{2} \rightarrow \alpha|\nabla w|^{2}$ in $L^{1}\left(\mathscr{R}\left(x_{0}, R, r\right)\right)$ as $t \rightarrow 0$. Consequently

$$
\lim _{t \rightarrow 0} \mu_{\alpha_{t}}\left(\mathscr{R}\left(x_{0}, R, r\right), \tilde{d}\right) \leq \mu_{\alpha}\left(\mathscr{R}\left(x_{0}, R, r\right), \tilde{d}\right) .
$$

On the other hand, let $w_{t}$ be a minimizer of $\mu_{\alpha}\left(\mathscr{R}\left(x_{0}, R, r\right), \tilde{d}\right)$ and let $t_{n} \downarrow 0$. Up to a subsequence, $w_{t_{n}} \rightarrow w_{0}$ in $H^{1}\left(\mathscr{R}\left(x_{0}, R, r\right)\right)$ as $n \rightarrow \infty$ and $\sqrt{\alpha_{t_{n}}} \nabla w_{t_{n}} \rightarrow \sqrt{\alpha} \nabla w_{0}$ in $L^{2}\left(\mathscr{R}\left(x_{0}, R, r\right)\right)$.

Since the class $\mathcal{I}:=\left\{w \in H^{1}\left(\mathscr{R}\left(x_{0}, R, r\right), \mathbb{S}^{1}\right) \mid \operatorname{deg}_{B\left(x_{0}, R\right)}(w)=\tilde{d}\right\}$ is closed under the $H^{1}$-weak convergence (see Appendix $\mathrm{A}$ or [16]), we obtain that $w_{0} \in \mathcal{I}$. Consequently, we have

$$
\liminf _{t \rightarrow 0} \mu_{\alpha_{t}}\left(\mathscr{R}\left(x_{0}, R, r\right), \tilde{d}\right) \geq \mu_{\alpha}\left(\mathscr{R}\left(x_{0}, R, r\right), \tilde{d}\right) .
$$

Thus the proof of (C.12) is complete.

Therefore, without loss of generality, we may assume that $\alpha$ is Lipschitz.

One may easily prove that if $R \leq 4 r$, then $\mu_{\alpha}^{\operatorname{Dir}}\left(\mathscr{R}\left(x_{0}, R, r\right), \tilde{d}\right) \leq 2 \tilde{d}^{2} \pi \ln 4$. Thus we assume that $R>4 r$. Clearly, it suffices to obtain the result for $\tilde{d}=1$ and $x_{0}=0$. 
Let $w$ be a global minimizer of $\mu_{\alpha}\left(\mathscr{R}\left(x_{0}, R / 2,2 r\right), 1\right)$. As explained Section $\mathrm{A}$ denoting $x /|x|=\mathrm{e}^{\imath \theta}$, one may write $w=\mathrm{e}^{\imath(\theta+\phi)}$ for some $\phi \in H^{2}\left(\mathscr{R}\left(x_{0}, R / 2,2 r\right), \mathbb{R}\right)$. Now we switch to polar coordinates.

Consider

$$
I=\left\{\left.\rho \in[2 r, R / 2]\left|\int_{0}^{2 \pi} \alpha\right| \nabla(\theta+\phi)\right|^{2}(\rho, \theta) \mathrm{d} \theta \leq \frac{1}{\rho^{2}} \int_{0}^{2 \pi} \alpha(\rho, \theta) \mathrm{d} \theta\right\} .
$$

Then $I$ is closed (since $\phi \in H^{2}$ ). On the other hand, $I$ is non empty, by the mean value theorem.

Let $r_{1}=\min I$ and $r_{2}=\max I$. We may assume that $\phi\left(r_{2}, 0\right)=0$ and $\phi\left(r_{1}, 0\right)=\theta_{0}$. We construct a test function:

$$
\phi^{\prime}(\rho, \theta)=\left\{\begin{array}{ll}
0 & \text { if } 2 r_{2} \leq \rho \leq R \\
\frac{2 r_{2}-\rho}{r_{2}} \phi\left(r_{2}, \theta\right) & \text { if } r_{2} \leq \rho \leq 2 r_{2} \\
\phi(\rho, \theta) & \text { if } r_{1} \leq \rho \leq r_{2} \\
\frac{2 \rho-r_{1}}{r_{1}} \phi\left(r_{1}, \theta\right)+2 \frac{r_{1}-\rho}{r_{1}} \theta_{0} & \text { if } r_{1} / 2 \leq \rho \leq r_{1} \\
\theta_{0} & \text { if } r \leq \rho \leq r_{1} / 2
\end{array} .\right.
$$

As explained in [21], there is $C$ depending only on $b$ s.t.

$$
\frac{1}{2} \int_{\mathscr{R}(0, R / 2,2 r)} \alpha\left(\left|\nabla\left(\theta+\phi^{\prime}\right)\right|^{2}-|\nabla(\theta+\phi)|^{2}\right) \leq C .
$$

Thus the result follows.

As a direct consequence of Proposition 45 (the two first assertions of the next proposition are direct), we have

Proposition 46. Let $\alpha \in L^{\infty}\left(\mathbb{R}^{2},\left[b^{2}, 1\right]\right), R>r_{1}>r>0, \tilde{d} \in \mathbb{Z}$ and $x_{0} \in \mathbb{R}^{2}$, we have

1. $\mu_{\alpha}\left(\mathscr{R}\left(x_{0}, R, r\right), \tilde{d}\right)=\tilde{d}^{2} \mu_{\alpha}\left(\mathscr{R}\left(x_{0}, R, r\right), 1\right)$,

2. $b^{2} \pi \ln \frac{R}{r} \leq \mu_{\alpha}\left(\mathscr{R}\left(x_{0}, R, r\right), 1\right) \leq \pi \ln \frac{R}{r}$,

3. $\mu_{\alpha}\left(\mathscr{R}\left(x_{0}, R, r\right), 1\right) \leq \mu_{\alpha}\left(\mathscr{R}\left(x_{0}, R, r_{1}\right), 1\right)+\mu_{\alpha}\left(\mathscr{R}\left(x_{0}, r_{1}, r\right), 1\right)+2 C_{b}$ where $C_{b}$ is given by Proposition 45 and depends only on $b$.

We turn to the construction of test functions in $R_{j}$ and $R_{j, k}$.

Using Proposition 45, there is $C_{b}$ depending only on $b \in(0,1)$ s.t. for $\alpha \in L^{\infty}\left(\Omega,\left[b^{2}, 1\right]\right)$ and for all $k \in\{1, \ldots, K-1\}, j \in\left\{1, \ldots, N_{k}\right\}$, there is $w_{\alpha, j, k} \in H^{1}\left(R_{j, k}, \mathbb{S}^{1}\right)$ s.t.

$$
w_{\alpha, j, k}(x)= \begin{cases}\frac{\left(x-x_{j}^{k}\right)^{\tilde{d}_{j, k}}}{\eta^{\prime} \tilde{d}_{j, k}} & \text { for } x \in \partial B\left(x_{j}^{k}, \eta_{k+1}^{\prime}\right) \\ \gamma_{\alpha, j, k} \frac{\left(x-x_{j}^{k}\right)^{\tilde{d}_{j, k}}}{\eta_{k}^{\tilde{d}_{j, k}}} & \text { for } x \in \partial B\left(x_{j}^{k}, \eta_{k}\right) \text { where } \gamma_{\alpha, j, k} \in \mathbb{S}^{1}\end{cases}
$$


and s.t. for all $w \in H^{1}\left(R_{j, k}, \mathbb{S}^{1}\right)$ satisfying $\operatorname{deg}_{\partial B\left(x_{j}^{k}, \eta_{k}\right)}(w)=\tilde{d}_{j, k}$ one has

$$
\int_{R_{j, k}} \alpha\left|\nabla w_{\alpha, j, k}\right|^{2} \leq \int_{R_{j, k}} \alpha|\nabla w|^{2}+C_{b} \tilde{d}_{j, k}^{2} \leq \int_{R_{j, k}} \alpha|\nabla w|^{2}+2 d^{2} C_{b} .
$$

Now we consider the rings $R_{j}$. For $j \in\left\{1, \ldots, N^{\prime}\right\}$, we denote

$$
\tilde{d}_{j}=\sum_{x_{i} \in B\left(y_{j}, \eta\right)} d_{i}
$$

Using Proposition 45, for $j \in\left\{1, \ldots, N^{\prime}\right\}$, we obtain $w_{\alpha, j} \in H^{1}\left(R_{j}, \mathbb{S}^{1}\right)$ s.t.

$$
w_{\alpha, j}(x)= \begin{cases}\frac{\left(x-y_{j}\right)^{d}}{\eta^{d}} & \text { for } x \in \partial B\left(y_{j}, \eta\right) \\ \gamma_{\alpha, j} \frac{\left(x-y_{j}\right)^{d}}{\eta_{K}^{d}} & \text { for } x \in \partial B\left(y_{j}, \eta_{K}\right) \text { where } \gamma_{\alpha, j} \in \mathbb{S}^{1}\end{cases}
$$

and s.t. for all $w \in H^{1}\left(R_{j}, \mathbb{S}^{1}\right)$ satisfying $\operatorname{deg}_{\partial B\left(y_{j}, \eta\right)}(w)=\tilde{d}_{j}$ one has

$$
\int_{R_{j}} \alpha\left|\nabla w_{\alpha, j}\right|^{2} \leq \int_{R_{j}} \alpha|\nabla w|^{2}+2 d^{2} C_{b} .
$$

\section{C.4 Proof of Proposition 10}

Note that there are at most $d^{2}$ regions $D_{j, k}$, at most $d^{2}$ rings $R_{j, k}$ and at most $d$ rings $R_{j}$. Consequently, denoting

$$
C_{4}\left(\eta_{\text {stop }}\right)=C_{1}\left(\eta_{\text {stop }}\right)+d^{2} C_{2}\left(2 \cdot 9^{d-1}, d\right)+4 d^{4} C_{b}
$$

and using (C.7), (C.8), (C.9), (C.14), (C.15), one may construct a test function $w_{\alpha} \in \mathcal{J}_{\rho}$ (up to multiply by some $\mathbb{S}^{1}$-Constants each function previously constructed) s.t. for all $w \in \mathcal{I}_{\rho}$, one has

$$
\int_{\Omega_{\rho}} \alpha\left|\nabla w_{\alpha}\right|^{2} \leq \int_{\Omega_{\rho}} \alpha|\nabla w|^{2}+C_{4}
$$

Clearly, (C.16) allows us to prove Proposition 10 with $C_{0}=C_{4} / 2$.

\section{Proof of Proposition 12}

\section{D.1 Description of the special solution $U_{\varepsilon}$}

From Proposition 3, we know that far away $\partial \omega_{\varepsilon}, U_{\varepsilon}$ is uniformly close to $a_{\varepsilon}$. Here we prove that, in a neighborhood of $\partial \omega_{\varepsilon}, U_{\varepsilon}$ is very close to a cell regularization of $a_{\varepsilon}$.

Let

$$
\begin{aligned}
& a^{\lambda}: Y=\left(-\frac{1}{2}, \frac{1}{2}\right) \times\left(-\frac{1}{2}, \frac{1}{2}\right) \rightarrow \begin{array}{c}
\{b, 1\} \\
x
\end{array} \\
& \mapsto \begin{cases}b & \text { if } x \in \omega^{\lambda}=\lambda \cdot \omega . \\
1 & \text { otherwise }\end{cases}
\end{aligned}
$$

Consider $V_{\xi}$ the unique minimizer of

$$
E_{\xi}^{a^{\lambda}}(V, Y)=\frac{1}{2} \int_{Y}|\nabla V|^{2}+\frac{1}{2 \xi^{2}}\left(a^{\lambda^{2}}-V^{2}\right)^{2}, V \in H_{1}^{1}(Y, \mathbb{R}) .
$$


Lemma 47. We have the existence of $C, \gamma>0$ s.t. for $\varepsilon>0$ and $x \in Y$

$$
\left|U_{\varepsilon}\left[y_{i, j}^{\varepsilon}+\delta^{j} x\right]-V_{\varepsilon / \delta^{j}}(x)\right| \leq C \mathrm{e}^{-\frac{\gamma \delta^{j}}{\varepsilon}} .
$$

Thus in the periodic case, we have $U_{\varepsilon}$ which is almost a $\delta \cdot(\mathbb{Z} \times \mathbb{Z})$-periodic function in $\Omega_{\delta}^{\text {incl }}$ in the sense that

$$
\left|U_{\varepsilon}(x)-U_{\varepsilon}[x+(\delta k, \delta l)]\right| \leq C \mathrm{e}^{-\frac{\gamma \delta}{\varepsilon}} \text { if } x, x+(\delta k, \delta l) \in \Omega_{\delta}^{\text {incl }} \text { and } k, l \in \mathbb{Z} .
$$

Proof. Step 1. We first prove that, for all $s>0$ and for sufficiently small $\varepsilon$, we have $U_{\varepsilon}^{2} \geq \frac{b^{2}+1}{2}-s$ in $\Omega \backslash \omega_{\varepsilon}$. The same argument leads to $U_{\varepsilon}^{2} \leq \frac{b^{2}+1}{2}+s$ in $\overline{\omega_{\varepsilon}}$ and for sufficiently small $\xi: V_{\xi}^{2} \geq \frac{b^{2}+1}{2}-s$ in $Y \backslash \omega^{\lambda}$ and $V_{\xi}^{2} \leq \frac{b^{2}+1}{2}+s$ in $\omega^{\lambda}$.

From Proposition 3 , it suffices to prove that for

$$
R=\alpha^{-1} \ln \frac{C}{1-\sqrt{\frac{1+b^{2}}{2}}},
$$

we have $U_{\varepsilon}^{2} \geq \frac{b^{2}+1}{2}-s$ in $\left\{x \in \Omega \backslash \omega_{\varepsilon} \mid \operatorname{dist}\left(x, \partial \omega_{\varepsilon}\right)<R \varepsilon\right\}$ (for sufficiently small $\varepsilon$ ). Here $C>1, \alpha>0$ are given by (1.5).

We fix $0<s<1$ and we let $z_{\varepsilon}=y_{i, j}^{\varepsilon}+\lambda \delta^{j} z_{\varepsilon}^{0} \in \partial \omega_{\varepsilon}, z_{\varepsilon}^{0} \in \partial \omega$. For $x \in B\left(z_{\varepsilon}, \lambda \delta^{P+1}\right)$, we write $x=z_{\varepsilon}+\varepsilon \tilde{x}$ with $\tilde{x} \in B\left(0, \lambda \delta^{P+1} / \varepsilon\right)$. Here $P=1$ and $y_{i, j}^{\varepsilon} \in \delta \mathbb{Z} \times \delta \mathbb{Z}$ if we are in the periodic situation.

We define

$$
\begin{aligned}
& \tilde{U}_{\varepsilon}(\tilde{x}): B\left(0, \lambda \delta^{P+1} / \varepsilon\right) \rightarrow \quad[b, 1] \\
& \tilde{x} \quad \mapsto U_{\varepsilon}\left(z_{\varepsilon}+\varepsilon \tilde{x}\right)
\end{aligned}
$$

It is easy to check that

$$
\left\{\begin{array}{l}
-\Delta \tilde{U}_{\varepsilon}=\tilde{U}_{\varepsilon}\left(\tilde{a}_{\varepsilon}^{2}-\tilde{U}_{\varepsilon}^{2}\right) \text { in } B\left(0, \lambda \delta^{P+1} / \varepsilon\right) \\
\tilde{U}_{\varepsilon} \in H^{1} \cap L^{\infty}\left(B\left(0, \lambda \delta^{P+1} / \varepsilon\right),[b, 1]\right)
\end{array}\right.
$$

where

$$
\tilde{a}_{\varepsilon}=\left\{\begin{array}{ll}
b & \text { in } \frac{\omega_{\varepsilon}-z_{\varepsilon}}{\varepsilon} \cap B\left(0, \lambda \delta^{P+1} / \varepsilon\right) \\
1 & \text { in } \frac{\left(\mathbb{R}^{2} \backslash \omega_{\varepsilon}\right)-z_{\varepsilon}}{\varepsilon} \cap B\left(0, \lambda \delta^{P+1} / \varepsilon\right)
\end{array} .\right.
$$

Clearly

$$
\begin{aligned}
\frac{\omega_{\varepsilon}-z_{\varepsilon}}{\varepsilon} \cap B\left(0, \lambda \delta^{P+1} / \varepsilon\right) & =\left[\frac{\lambda \delta^{j}}{\varepsilon} \cdot\left(\omega-z_{\varepsilon}^{0}\right)\right] \cap B\left(0, \lambda \delta^{P+1} / \varepsilon\right) \\
& =\frac{\lambda \delta^{j}}{\varepsilon} \cdot\left[\left(\omega-z_{\varepsilon}^{0}\right) \cap B\left(0, \delta^{P+1-j}\right)\right]
\end{aligned}
$$

and thus

$$
\left.\frac{\left(\mathbb{R}^{2} \backslash \omega_{\varepsilon}\right)-z_{\varepsilon}}{\varepsilon} \cap B\left(0, \lambda \delta^{P+1} / \varepsilon\right)=\frac{\lambda \delta^{j}}{\varepsilon} \cdot\left[\left(\mathbb{R}^{2} \backslash \omega\right)-z_{\varepsilon}^{0}\right) \cap B\left(0, \delta^{P+1-j}\right)\right] .
$$

Note that $\lambda \delta^{P+1} / \varepsilon \rightarrow \infty$ and $\delta^{P+1-j} \rightarrow 0$, thus by smoothness of $\omega$, up to a subsequence, we have $\frac{\lambda \delta^{j}}{\varepsilon} \cdot\left\{\left[\left(\mathbb{R}^{2} \backslash \omega\right)-z_{\varepsilon}^{0}\right] \cap B\left(0, \delta^{P+1-j}\right)\right\} \rightarrow \mathcal{R}_{\theta_{0}}\left(\mathbb{R} \times \mathbb{R}^{+}\right)$. Here $\mathcal{R}_{\theta_{0}}$ is the vectorial rotation of angular $\theta_{0} \in[0,2 \pi)$. 
For sake of simplicity, we assume that $\theta_{0}=0$.

From (D.2) and standard elliptic estimates, we obtain that $\tilde{U}_{\varepsilon}$ is bounded in $W^{2, p}(B(0, R))$ for $p \geq 2, R>0$. Thus up to consider a subsequence, we obtain that $\tilde{U}_{\varepsilon} \rightarrow \tilde{U}_{b}$ in $C_{\text {loc }}^{1}\left(\mathbb{R}^{2}\right)$ $(\varepsilon \rightarrow 0)$ where $\tilde{U}_{b} \in C^{1}\left(\mathbb{R}^{2},[b, 1]\right)$ is a solution of

$$
\begin{cases}-\Delta \tilde{U}_{b}=\tilde{U}_{b}\left(1-\tilde{U}_{b}^{2}\right) & \text { in } \mathbb{R} \times \mathbb{R}^{+} \\ -\Delta \tilde{U}_{b}=\tilde{U}_{b}\left(b^{2}-\tilde{U}_{b}^{2}\right) & \text { in } \mathbb{R} \times \mathbb{R}^{-} . \\ \tilde{U}_{b} \in C^{1}\left(\mathbb{R}^{2}\right) \cap H_{\text {loc }}^{2}\left(\mathbb{R}^{2}\right) \cap L^{\infty}\left(\mathbb{R}^{2}\right) & \end{cases}
$$

It is proved in [15] (Theorem 2.2), that (D.3) admits a unique positive solution. Moreover $\tilde{U}_{b}(x, y)=U_{b}(y)\left(\tilde{U}_{b}\right.$ is independent of its first variable) and $U_{b}$ is the unique solution of

$$
\left\{\begin{array}{ll}
-U_{b}^{\prime \prime}=U_{b}\left(1-U_{b}^{2}\right) & \text { in } \mathbb{R}^{+} \\
-U_{b}^{\prime \prime}=U_{b}\left(b^{2}-U_{b}^{2}\right) & \text { in } \mathbb{R}^{-} \\
U_{b} \in C^{1}(\mathbb{R}, \mathbb{R}), U_{b}^{\prime}>0, & \lim _{+\infty} U_{b}=1, \lim _{-\infty} U_{b}=b
\end{array} .\right.
$$

Note that since the limit is unique, the convergence is valid for the whole sequence.

This solution $U_{b}$ may be explicitly obtained by looking for $U_{b}$ under the form

$$
U_{b}(x)=\left\{\begin{array}{ll}
\frac{A \mathrm{e}^{\sqrt{2} x}-1}{A \mathrm{e}^{\sqrt{2} x}+1} & \text { if } x \geq 0 \\
b \frac{B \mathrm{e}^{-b \sqrt{2} x}-1}{B \mathrm{e}^{-b \sqrt{2} x}+1} & \text { if } x<0
\end{array} .\right.
$$

We get $B=-\frac{3 b^{2}+1+2 b \sqrt{2\left(b^{2}+1\right)}}{1-b^{2}}, A=\frac{B(1+b)+1-b}{B(1-b)+1+b}$ and

$$
U_{b}(0)=b \frac{B-1}{B+1}=\frac{1+b^{2}+b \sqrt{2\left(b^{2}+1\right)}}{2 b+\sqrt{2\left(b^{2}+1\right)}}=\frac{1-b^{2}}{2 b+\sqrt{2\left(b^{2}+1\right)}}+b=\sqrt{\frac{b^{2}+1}{2}}
$$

Since $U_{b}(0)^{2}=\frac{b^{2}+1}{2}$ and $U_{b}$ is an increasing function, for $x \geq 0, U_{b}(x)^{2} \geq \frac{b^{2}+1}{2}$. From the convergence $\tilde{U}_{\varepsilon} \rightarrow \tilde{U}_{b}$ in $L^{\infty}(B(0, R))$, we obtain that, for $\varepsilon$ sufficiently small, $\tilde{U}_{\varepsilon}^{2} \geq \frac{b^{2}+1}{2}-s$ in $B(0, R) \cap\left\{\frac{\lambda \delta^{j}}{\varepsilon} \cdot\left[\left(\mathbb{R}^{2} \backslash \omega\right)-z_{\varepsilon}^{0}\right]\right\}$.

Step 2. Fix $j \in\{1, \ldots, P\}$ s.t. $\mathcal{M}_{j}^{\varepsilon} \neq \emptyset$ and fix $i \in \mathcal{M}_{j}^{\varepsilon}$. Note that if we are in the periodic case then $j=1$ and we fix $y_{k, l}=(\delta k, \delta l) \in \delta \mathbb{Z} \times \delta \mathbb{Z}$ s.t. $y_{k, l}+\delta \cdot Y \subset \Omega$.

We denote $\xi:=\frac{\delta^{j}}{\varepsilon}$. For $x \in Y$, consider $W(x)=V_{\xi}(x)-U_{\varepsilon}\left(y_{i, j}^{\varepsilon}+\delta^{j} x\right)$ which satisfies (using (1.5))

$$
\left\{\begin{array}{ll}
-\xi^{2} \Delta W(x)=W(x)\left\{a^{\lambda}(x)^{2}-\left[V_{\xi}(x)^{2}+U_{\varepsilon}\left(y_{i, j}^{\varepsilon}+\delta^{j} x\right) V_{\xi}(x)+U_{\varepsilon}\left(y_{i, j}^{\varepsilon}+\delta^{j} x\right)^{2}\right]\right\} & \text { in } Y \\
0 \leq W \leq C \mathrm{e}^{-\frac{\gamma}{\xi}} & \text { on } \partial Y
\end{array} .\right.
$$

Here $\gamma=\alpha \cdot \operatorname{dist}(\partial Y, \omega), C$ and $\alpha$ given by (1.5).

By Step 1, taking $s=b^{2}$, for sufficiently small $\varepsilon$, we have for $x \in Y \backslash \omega^{\lambda}$

$$
U_{\varepsilon}^{2}\left(y_{i, j}^{\varepsilon}+\delta^{j} x\right), V_{\xi}^{2}(x) \geq \max \left(b^{2}, \frac{1-b^{2}}{2}\right) \geq \frac{1}{3} .
$$

Thus, using the weak maximum principle, we find that $W \geq 0$ in $Y$. Consequently, since $W$ is subharmonic, we deduce that $W \leq C \mathrm{e}^{-\frac{\gamma}{\xi}}$. 


\section{D.2 Behavior of almost minimizers of $I_{\rho, \varepsilon}$}

We recall that for $x_{0} \in \mathbb{R}^{2}$ and $R>r>0$, we denoted $\mathscr{R}\left(x_{0}, R, r\right):=B\left(x_{0}, R\right) \backslash$ $\overline{B\left(x_{0}, r\right)}$.

\section{D.2.1 Useful results for the periodic situation}

We establish three preliminary results for the periodic situation represented Figure 1 . Thus in this subsection we assume that $U_{\varepsilon}$ is the unique global minimizer of $E_{\varepsilon}$ in $H_{1}^{1}$ with the periodic pinning term $a_{\varepsilon}$ represented Figure 1 .

\section{Energetic estimates in rings and global energetic upper bounds}

From Lemma 47 ( $U_{\varepsilon}$ is close to a periodic function) we obtain

Lemma 48. For all $1 \geq R>r \geq \varepsilon, x, x_{0} \in \mathbb{R}^{2}$ s.t. $B\left(x_{0}, R\right) \subset \Omega_{\delta}^{\text {incl }}$ and $x-x_{0} \in \delta \cdot \mathbb{Z}^{2}$, we have

$$
\mu_{\varepsilon}(\mathscr{R}(x, R, r), 1) \geq \mu_{\varepsilon}\left(\mathscr{R}\left(x_{0}, R, r\right), 1\right)-o_{\varepsilon}(1) .
$$

Adding the condition that $B(x, R) \subset \Omega_{\delta}^{\text {incl }}$, we have

$$
\left|\mu_{\varepsilon}(\mathscr{R}(x, R, r), 1)-\mu_{\varepsilon}\left(\mathscr{R}\left(x_{0}, R, r\right), 1\right)\right| \leq o_{\varepsilon}(1) .
$$

Moreover the $o_{\varepsilon}(1)$ may be considered independent of $x, x_{0}, R, r$.

Lemma 48 implies easily the following estimate.

Proposition 49. Let $\eta>0$ and $\eta>\rho \geq \varepsilon$. Then there is $C=C\left(\Omega, \Omega^{\prime}, g, b, \eta\right)>0$ s.t. for $x_{0} \in \mathbb{R}^{2}$ we have

$$
I_{\rho, \varepsilon} \leq d \mu_{\varepsilon}\left(\mathscr{R}\left(x_{0}, \eta, \rho\right), 1\right)+C(\eta),
$$

where $C(\eta)$ is independent of $x_{0}$ and $\rho$.

From Lemma 47 we get the almost periodicity of $\mu_{\varepsilon}(\mathscr{R}(\cdot, R, r), 1)$ w.r.t. a $\delta \times \delta$-grid (expressed in Lemma 47). Therefore, the "best points" to minimize $\mu_{\varepsilon}(\mathscr{R}(\cdot, R, r), 1)$ should be almost periodic.

Another important result is the next proposition. It expresses that the center of an inclusion is not too far to a good point to minimize $\mu_{\varepsilon}(\mathscr{R}(\cdot, R, r), 1)$. This proposition may be seen as a first step in the proof of the pinning effect of $\omega_{\varepsilon}$.

Proposition 50. There is $C_{*}$ which depends only on $\omega, b$ and $\Omega$ s.t. for sufficiently small $\varepsilon$, for $x \in \Omega$ and $x_{\text {per }} \in B(x, 3 \delta \sqrt{2} / 2) \cap(\delta \mathbb{Z} \times \delta \mathbb{Z}) \cap \omega_{\varepsilon}$ we have for $1>R>r>\varepsilon$

$$
\mu_{\varepsilon}\left(\mathscr{R}\left(x_{\mathrm{per}}, R, r\right), 1\right) \leq \mu_{\varepsilon}(\mathscr{R}(x, R, r), 1)+C_{*} .
$$

Proof. If $R \leq 10^{2} r$, then the result is obvious with $C_{*}=2 \pi \ln 10$. Thus we assume that $R>10^{2} r$.

We share the proof in three cases:

Case 1. $r \geq \delta$,

Case 2. $\delta \geq R>r \geq \lambda \delta$,

Case 3. $R \leq \lambda \delta$. 
Assume for the moment that

There exists $\tilde{C}_{*}>0$ s.t. (D.4) yields in the three previous cases with $C_{*}=\tilde{C}_{*}$.

For the general case, we divide $\mathscr{R}(x, R, r)$ into $R_{1}(x) \cup R_{2}(x) \cup R_{3}(x)$ with

$$
\begin{gathered}
R_{1}(x)=\mathscr{R}(x, R, \min \{\max (\delta, r), R\}), \\
R_{2}(x)=\mathscr{R}(x, \min \{\max (\delta, r), R\}, \min \{R, \max (\lambda \delta, r)\}), \\
R_{3}(x)=\mathscr{R}(x, \min \{R, \max (\lambda \delta, r)\}, r) .
\end{gathered}
$$

Remark 51. 1. For $k \in\{1,2,3\}$, we have $\emptyset \subseteq R_{k} \subseteq \mathscr{R}(x, R, r)$.

2. It is easy to check that

- $\left[R_{1}=\mathscr{R}(x, R, r) \Leftrightarrow r \geq \delta\right.$ (Case 1.)] and $\left[R_{1}=\emptyset \Leftrightarrow R \leq \delta\right]$,

- $\left[R_{2}=\mathscr{R}(x, R, r) \Leftrightarrow \lambda \delta \leq r<R \leq \delta\right.$ (Case 2.)] and $\left[R_{2}=\emptyset \Leftrightarrow\{\lambda=1\right.$ or $r \geq \delta$ or $\left.R \leq \lambda \delta\}\right]$,

- $\left[R_{3}=\mathscr{R}(x, R, r) \Leftrightarrow R \leq \lambda \delta\right.$ (Case 3.)] and $\left[R_{3}=\emptyset \Leftrightarrow r \geq \lambda \delta\right]$.

3. If $\lambda \equiv 1$ then Case 2. never occurs and $R_{2}=\emptyset$.

Therefore we have (using Propositions 45, 46, 3 and (D.5))

$$
\begin{aligned}
\mu_{\varepsilon}(\mathscr{R}(x, R, r), 1) & \geq \mu_{\varepsilon}\left(R_{1}(x), 1\right)+\mu_{\varepsilon}\left(R_{2}(x), 1\right)+\mu_{\varepsilon}\left(R_{3}(x), 1\right) \\
(\text { D.5) } & \geq \mu_{\varepsilon}\left(R_{1}\left(x_{\text {per }}\right), 1\right)+\mu_{\varepsilon}\left(R_{2}\left(x_{\text {per }}\right), 1\right)+\mu_{\varepsilon}\left(R_{3}\left(x_{\text {per }}\right), 1\right)-3 \tilde{C}_{*} \\
(\text { Prop. 45) } & \geq \mu_{\varepsilon}^{\text {Dir }}\left(R_{1}\left(x_{\text {per }}\right), 1\right)+\mu_{\varepsilon}^{\text {Dir }}\left(R_{2}\left(x_{\text {per }}\right), 1\right)+\mu_{\varepsilon}^{\text {Dir }}\left(R_{3}\left(x_{\text {per }}\right), 1\right)-3\left(\tilde{C}_{*}+C_{b}\right) \\
& \geq \mu_{\varepsilon}\left(\mathscr{R}\left(x_{\text {per }}, R, r\right), 1\right)-3\left(\tilde{C}_{*}+C_{b}\right) .
\end{aligned}
$$

The last line is obtained by constructing a test function. Therefore, it suffices to take $C_{*}:=3\left(\tilde{C}_{*}+C_{b}\right)$.

We now turn to the proof of (D.5) in Case 1,2 and 3. Recall that we assumed that $R>10^{2} r$.

We treat Case $1 .(R>r \geq \delta)$ :

$$
\begin{aligned}
\mu_{\varepsilon}(\mathscr{R}(x, R, r), 1) \geq(\text { Prop. 45) } & \geq \mu_{\varepsilon}^{\operatorname{Dir}}(\mathscr{R}(x, R, r), 1)-C_{b} \\
& \geq \mu_{\varepsilon}^{\operatorname{Dir}}\left(\mathscr{R}\left(x, 10 R, 10^{-1} r\right), 1\right)-2 \pi \ln 10-C_{b} \\
\left\{\mathscr{R}\left(x_{\text {per }}, R, 10 r\right) \subset \mathscr{R}\left(x, 10 R, 10^{-1} r\right)\right\} & \geq \mu_{\varepsilon}\left(\mathscr{R}\left(x_{\text {per }}, R, 10 r\right), 1\right)-2 \pi \ln 10-C_{b} \\
\geq \text { (Prop. 45) } & \geq \mu_{\varepsilon}\left(\mathscr{R}\left(x_{\text {per }}, R, r\right), 1\right)-3 \pi \ln 10-2 C_{b} .
\end{aligned}
$$

Thus we may take $\tilde{C}_{*}=3 \pi \ln 10+2 C_{b}$.

We treat Case 2. Note that from Remark 51.3, we may assume that $\lambda \rightarrow 0$. On the one hand, it is clear that

$$
\mu_{\varepsilon}\left(\mathscr{R}\left(x_{\text {per }}, R, r\right), 1\right) \leq \pi \ln \frac{R}{r}
$$

On the other hand, letting

$$
\begin{aligned}
& \alpha_{\varepsilon}: \mathbb{R}^{2} \rightarrow \quad\left\{b^{2}, 1\right\} \\
& x \mapsto \begin{cases}b^{2} & \text { if } x \in \cup_{M \in \mathbb{Z}^{2}} \overline{B(\delta M, \lambda \delta)}, \\
1 & \text { otherwise }\end{cases}
\end{aligned}
$$

we have from Proposition 3 that $\alpha_{\varepsilon} \leq U_{\varepsilon}^{2}+V_{\varepsilon}$ with $\left\|V_{\varepsilon}\right\|_{L^{\infty}}=o\left(\varepsilon^{2}\right)$. 
If $\mathscr{R}(x, R, r) \cap\left\{\alpha_{\varepsilon}=b^{2}\right\}=\emptyset$, then we have $\mu_{\varepsilon}(\mathscr{R}(x, R, r), 1) \geq \pi \ln \frac{R}{r}+o\left(\varepsilon^{2} \ln \lambda\right)$. And thus the result holds with $\tilde{C}_{*}=1$ (for sufficiently small $\varepsilon$ ).

Otherwise we have $\mathscr{R}(x, R, r) \cap\left\{\alpha_{\varepsilon}=b^{2}\right\} \neq \emptyset$. In this situation, because $R \leq \delta$, we get that $\mathscr{R}(x, R, r) \cap\left\{\alpha_{\varepsilon}=b^{2}\right\}$ is a union of at most four connected components. Therefore $S=\left\{\rho \in(r, R) \mid \partial B(x, \rho) \cap\left\{\alpha_{\varepsilon}=b^{2}\right\}\right\}$ is a union of at most four segments whose length is lower than $8 \lambda \delta$. Consequently, denoting $\bar{S}=\cup_{i=1}^{k}\left[s_{i}, t_{i}\right]$ (with $s_{i}<s_{i+1}$ ), we have for $w_{*} \in H^{1}\left(\mathscr{R}(x, R, r), \mathbb{S}^{1}\right)$ which minimizes $\mu_{\varepsilon}(\mathscr{R}(x, R, r), 1)$

$$
\begin{aligned}
\frac{1}{2} \int_{\mathscr{R}(x, R, r)} U_{\varepsilon}^{2}\left|\nabla w_{*}\right|^{2}+o_{\varepsilon}(1) & \geq \frac{1}{2} \int_{\mathscr{R}(x, R, r)} \alpha_{\varepsilon}\left|\nabla w_{*}\right|^{2} \\
\left(t_{0}=r \& s_{k+1}=R\right) & \geq \sum_{i=0}^{k} \frac{1}{2} \int_{t_{i}}^{s_{i+1}} \frac{\mathrm{d} \rho}{\rho} \int_{0}^{2 \pi}\left|\partial_{\theta} w_{*}\right|^{2} \\
& \geq \pi \sum_{i=0}^{k} \ln \frac{s_{i+1}}{t_{i}}=\pi \ln \frac{R}{r}-\pi \sum_{i=1}^{k} \ln \frac{t_{i}}{s_{i}}
\end{aligned}
$$

Since $\lambda \delta \leq s_{i} \leq t_{i} \leq s_{i}+8 \lambda \delta$, we have $1 \leq \frac{t_{i}}{s_{i}} \leq 1+\frac{8 \lambda \delta}{s_{i}} \leq 9$. Therefore we may take $\tilde{C}_{*}=4 \pi \ln 9+1$.

We treat the last case. Since $R \leq \lambda \delta$ and $x_{\text {per }} \in(\delta \mathbb{Z} \times \delta \mathbb{Z}) \cap \omega_{\varepsilon}$, there is $\tilde{C}_{*}$ s.t. we have (for sufficiently small $\varepsilon) \mu_{\varepsilon}\left(\mathscr{R}\left(x_{\mathrm{per}}, R, r\right), 1\right) \leq \pi b^{2} \ln \frac{R}{r}+\tilde{C}_{*}$. On the other hand (Proposition 46,2) we have $\mu_{\varepsilon}(\mathscr{R}(x, R, r), 1) \geq \pi b^{2} \ln \frac{R}{r}$. Therefore the estimate in the third case is proved.

\section{Estimates for almost minimizers}

In this subsection we establish a fundamental result: fix an almost minimal configuration $\{\mathbf{x}, \mathbf{1}\}$ for $I_{\rho, \varepsilon}$ (the existence of such configuration is proved Section D.3) and a map which almost minimizes $\frac{1}{2} \int_{\Omega^{\prime} \backslash \cup \overline{B\left(x_{i}, \rho\right)}} U_{\varepsilon}^{2}|\nabla \cdot|^{2}$. Then the map almost minimizes the weighted Dirichlet functional $\frac{1}{2} \int_{\mathscr{R}\left(x_{i}, \rho^{\prime}, \rho\right)} U_{\varepsilon}^{2}|\nabla \cdot|^{2}, 10^{-2} \min _{i \neq j}\left|x_{i}-x_{j}\right|>\rho^{\prime}>\rho$.

Lemma 52. 1. Let $x \in \mathbb{R}^{2}, 0<r<R, \alpha \in L^{\infty}\left(\mathbb{R}^{2},\left[b^{2}, 1\right]\right), C_{0}>0$ and a map $w \in H^{1}\left(\mathscr{R}(x, R, r), \mathbb{S}^{1}\right)$ s.t. $\operatorname{deg}_{\partial B(x, R)}(w)=1$ and

$$
\frac{1}{2} \int_{\mathscr{R}(x, R, r)} \alpha|\nabla w|^{2}-\mu_{\alpha}(\mathscr{R}(x, R, r), 1) \leq C_{0} .
$$

Then for all $r^{\prime}, R^{\prime}$ s.t. $r<r^{\prime}<R^{\prime}<R$ one has

$$
\frac{1}{2} \int_{\mathscr{R}\left(x, R^{\prime}, r^{\prime}\right)} \alpha|\nabla w|^{2}-\mu_{\alpha}\left(\mathscr{R}\left(x, R^{\prime}, r^{\prime}\right), 1\right) \leq 4 C_{b}+C_{0},
$$

where $C_{b}$ depends only on $b$ and is given by Proposition 45 .

2. Let $x_{1}, \ldots, x_{d} \in \Omega\left(x_{i} \neq x_{j}\right.$ for $\left.i \neq j\right), d_{i}=1, \varepsilon<\rho<10^{-2} \eta, \eta:=10^{-2}$. $\min \left\{\left|x_{i}-x_{j}\right|, \operatorname{dist}\left(x_{i}, \partial \Omega\right)\right\}, C_{0}>0$ and $w \in H^{1}\left(\Omega_{\rho}^{\prime}, \mathbb{S}^{1}\right)$ s.t.

$$
\frac{1}{2} \int_{\Omega_{\rho}^{\prime}} U_{\varepsilon}^{2}|\nabla w|^{2} \leq I_{\rho, \varepsilon}+C_{0} .
$$


Then for $\rho \leq r<R<\eta$ one has for all $i$

$$
\frac{1}{2} \int_{\mathscr{R}\left(x_{i}, R, r\right)} U_{\varepsilon}^{2}|\nabla w|^{2}-\mu_{\varepsilon}\left(\mathscr{R}\left(x_{i}, R, r\right), 1\right) \leq C_{0}+C(\eta)
$$

here $C(\eta)$ depends only on $b, g, \Omega, \Omega^{\prime}$ and $\eta$.

3. Under the hypotheses of 2., we also have for $\eta>\rho_{0}>\rho$

$$
\frac{1}{2} \int_{\Omega_{\rho_{0}}^{\prime}} U_{\varepsilon}^{2}|\nabla w|^{2} \leq C\left(\rho_{0}, C_{0}\right)
$$

here $C\left(\rho_{0}, C_{0}\right)$ depends only on $b, g, \Omega, \Omega^{\prime}, C_{0}, \rho_{0}$ and $\eta$.

Proof. Using the third part of Proposition 46, we have

$$
\begin{aligned}
\frac{1}{2} \int_{\mathscr{R}(x, R, r)} \alpha|\nabla w|^{2} \leq \mu_{\alpha}(\mathscr{R}(x, R, & \left.\left.R^{\prime}\right), 1\right)+\mu_{\alpha}\left(\mathscr{R}\left(x, R^{\prime}, r^{\prime}\right), 1\right) \\
& +\mu_{\alpha}\left(\mathscr{R}\left(x, r^{\prime}, r\right), 1\right)+4 C_{b}+C_{0} .
\end{aligned}
$$

We easily obtain

$$
\frac{1}{2} \int_{\mathscr{R}(x, R, r)} \alpha|\nabla w|^{2} \geq \mu_{\alpha}\left(\mathscr{R}\left(x, R, R^{\prime}\right), 1\right)+\frac{1}{2} \int_{\mathscr{R}\left(x, R^{\prime}, r^{\prime}\right)} \alpha|\nabla w|^{2}+\mu_{\alpha}\left(\mathscr{R}\left(x, r^{\prime}, r\right), 1\right)
$$

which proves the first assertion.

The second assertion is obtained by using the same argument combined with Proposition 49

Last assertion is a straightforward consequence of Proposition 49 and both previous assertions.

\section{D.2.2 Lower bound on circles}

In this subsection we prove an estimate for the minimization of weighted 1-dimensional Dirichlet functionals. In the following this estimate will be used to get lower bounds in rings.

Lemma 53. Let $\theta_{0} \in(0,2 \pi)$ and let $\alpha \in L^{\infty}\left([0,2 \pi],\left\{b^{2}, 1\right\}\right)$ be s.t. $\mathscr{H}^{1}\left(\left\{\alpha=b^{2}\right\}\right)=\theta_{0}$. Let $\varphi \in H^{1}([0,2 \pi], \mathbb{R})$ s.t. $\varphi(2 \pi)-\varphi(0)=2 \pi$. The following lower bound holds

$$
\frac{1}{2} \int_{0}^{2 \pi} \alpha(\theta)\left|\partial_{\theta} \varphi(\theta)\right|^{2} \mathrm{~d} \theta \geq \frac{2 \pi^{2}}{\int_{0}^{2 \pi} \frac{1}{\alpha}}=\frac{2 \pi^{2}}{2 \pi+\theta_{0}\left(b^{-2}-1\right)} .
$$

Here $\mathscr{H}^{1}$ is the 1-dimensional Hausdorff measure.

Proof. The proof of this lower bound is based on the computation of the minimal energy.

It is easy to check that a minimal function $\varphi_{\min } \in H^{1}([0,2 \pi], \mathbb{R})$ for $\frac{1}{2} \int_{0}^{2 \pi} \alpha(\theta)\left|\partial_{\theta} \cdot\right|^{2} \mathrm{~d} \theta$ under the constraint $\varphi(2 \pi)-\varphi(0)=2 \pi$ exists and satisfies $\partial_{\theta}\left(\alpha \partial_{\theta} \varphi_{\min }\right)=0$. Thus $\partial_{\theta} \varphi_{\min }=\frac{\text { Cst }}{\alpha}$ with $\mathrm{Cst}=\frac{2 \pi}{\int_{0}^{2 \pi} \alpha^{-1}}$. Therefore

$$
\frac{1}{2} \int_{0}^{2 \pi} \alpha(\theta)\left|\partial_{\theta} \varphi(\theta)\right|^{2} \mathrm{~d} \theta \geq \frac{1}{2} \int_{0}^{2 \pi} \alpha(\theta)\left|\partial_{\theta} \varphi_{\min }(\theta)\right|^{2} \mathrm{~d} \theta=\frac{2 \pi^{2}}{\int_{0}^{2 \pi} \frac{1}{\alpha}}=\frac{2 \pi^{2}}{2 \pi+\theta_{0}\left(b^{-2}-1\right)}
$$




\section{D.3 Proof of the first part of Proposition 12}

Let $x_{1}^{n}, \ldots, x_{N}^{n} \in \Omega$ s.t. $\left|x_{i}^{n}-x_{j}^{n}\right| \geq 8 \rho$ and $d_{1}, \ldots, d_{N}>0, \sum d_{i}=d$ (up to a subsequence the degrees may be considered independent of $n$ ).

Assume that

$$
\text { there is } i_{0} \in\{1, \ldots, N\} \text { s.t. } d_{i_{0}} \neq 1 \text { or there are } i \neq j \text { s.t. }\left|x_{i}^{n}-x_{j}^{n}\right| \rightarrow 0 \text {. }
$$

Up to pass to a subsequence, there are $a_{1}, \ldots, a_{M} \in \bar{\Omega}$ and $\left\{\Lambda_{1}, \ldots, \Lambda_{M}\right\}$ a partition of $\{1, \ldots, N\}$ s.t.

$$
i \in \Lambda_{l} \Longleftrightarrow x_{i}^{n} \rightarrow a_{l} .
$$

For sake of simplicity, we drop the superscript $n$ for the points, i.e., we write $x_{i}$ instead of $x_{i}^{n}$.

We let $\rho_{0}:=10^{-2} \min \left\{\min _{k \neq l}\left|a_{k}-a_{l}\right|, \operatorname{dist}\left(\partial \Omega, \partial \Omega^{\prime}\right)\right\}$ with $\min _{k \neq l}\left|a_{k}-a_{l}\right|=+\infty$ if $M=1$.

Note that since $d_{i}>0$, (D.6) is equivalent to

$$
\text { there exists } l_{0} \in\{1, \ldots, M\} \text { s.t. } \tilde{d}_{l_{0}}=\sum_{i \in \Lambda_{l_{0}}} d_{i}>1 \text {. }
$$

We are going to prove that (D.7) is not possible for almost minimal configurations. In order to do this, for $l \in\{1, \ldots, M\}$, we obtain a lower bound for the weighted Dirichlet functional defined around $a_{l}$. Then using Proposition 49 we will conclude.

For $l \in\{1, \ldots, M\}$, there are two cases:

1. $\operatorname{Card}\left(\Lambda_{l}\right)>1$,

2. $\operatorname{Card}\left(\Lambda_{l}\right)=1$.

In the first case $\left(\operatorname{Card}\left(\Lambda_{l}\right)>1\right)$, we apply the separation process (defined Section C.1) in $\Omega_{l}^{n}=B\left(a_{l}, 2 \rho_{0}\right) \backslash \cup_{i \in \Lambda_{l}} \overline{B\left(x_{i}, \rho\right)}$ with $\eta_{\text {stop }}=10^{-2} \rho_{0}$.

By construction, the process stops after $K$ steps. For $k \in\{1, \ldots, K\}$ we denote:

- $\left\{x_{1}^{k}, \ldots, x_{N_{k}}^{k}\right\}$ the selection of points made in Step $k\left(x_{i}^{0}=x_{i}, i \in \Lambda_{l}\right)$,

- $\eta_{k}^{\prime}$ the radius of the intermediate balls in Step $k\left(\eta_{k}^{\prime}=\frac{1}{4} \min _{i \neq j}\left|x_{i}^{k-1}-x_{j}^{k-1}\right|\right)$,

- $\eta_{k}$ the radius of the final balls in Step $k\left(\eta_{k}=2 \kappa_{k} \eta_{k}^{\prime}, \kappa_{k} \in\left\{9^{0}, \ldots, 9^{d}\right\}\right.$ and $\left.\eta_{0}=\rho\right)$.

Since for $i, j \in \Lambda_{l}$ we have $\left|x_{i}-x_{j}\right| \rightarrow 0$, then, in the end of the process (after $K$ steps), we obtain a unique $x_{1}^{K}=y_{l} \in\left\{x_{i} \mid i \in \Lambda_{l}\right\}$ in the final selection of points and $\eta_{K} \rightarrow 0$.

From (C.3) and (C.4), the following rings are mutually disjoint (denoting $\eta_{0}=\rho$ )

$R_{0}^{l}=\mathscr{R}\left(y_{l}, \rho_{0}, \eta_{K}\right)$ and $R_{j, k}=\mathscr{R}\left(x_{j}^{k}, \eta_{k+1}^{\prime}, \eta_{k}\right)$ for $k \in\{0, \ldots, K-1\}, j \in\left\{1, \ldots, N_{k}\right\}$.

We let

- for $k \in\{0, \ldots, K-1\}$ and $j \in\left\{1, \ldots, N_{k}\right\}, \tilde{d}_{j, k}:=\sum_{x_{i} \in B\left(x_{j}^{k}, \eta_{k+1}^{\prime}\right)} d_{i}$,

- for $n \geq 1$ we let $x_{0}=x_{0}(n) \in(\delta \mathbb{Z} \times \delta \mathbb{Z}) \cap \omega_{\varepsilon_{n}}$ be s.t. $B\left(x_{0}, 2 \rho_{0}\right) \subset \Omega$. Thus combining Lemma 48 with Proposition 50, we get that (for sufficiently large $n$ ) and for $\rho_{0} \geq R>r \geq \rho$

$$
\mu_{\varepsilon_{n}}\left(\mathscr{R}\left(x_{0}, R, r\right), 1\right) \leq \inf _{x \in \Omega} \mu_{\varepsilon_{n}}(\mathscr{R}(x, R, r), 1)+C_{*}+1 .
$$


For $w \in H_{g}^{1}\left(\Omega_{\rho}^{\prime}, \mathbb{S}^{1}\right)$ we have

$$
\begin{aligned}
\frac{1}{2} \int_{\Omega_{l}^{n}} U_{\varepsilon_{n}}^{2}|\nabla w|^{2} & \geq \frac{1}{2} \int_{R_{0}^{l}} U_{\varepsilon_{n}}^{2}|\nabla w|^{2}+\sum_{k=0}^{K-1} \sum_{j=1}^{N_{k}} \frac{1}{2} \int_{R_{j, k}} U_{\varepsilon_{n}}^{2}|\nabla w|^{2} \\
& \geq \frac{1}{2} \int_{R_{0}^{l}} U_{\varepsilon_{n}}^{2}|\nabla w|^{2}+\sum_{k=0}^{K-1} \sum_{j=1}^{N_{k}} \mu_{\varepsilon_{n}}\left(\mathscr{R}\left(x_{j}^{k}, \eta_{k+1}^{\prime}, \eta_{k}\right), \tilde{d}_{j, k}\right) \\
(\underline{\mathrm{D} .8}) & \geq \frac{1}{2} \int_{\mathscr{R}\left(x_{0}, \rho_{0}, \eta_{K}\right)} U_{\varepsilon_{n}}^{2}|\nabla w|^{2}+\sum_{k=0}^{K-1} \sum_{j=1}^{N_{k}} \mu_{\varepsilon_{n}}\left(\mathscr{R}\left(x_{0}, \eta_{k+1}^{\prime}, \eta_{k}\right), \tilde{d}_{j, k}\right)-\mathcal{O}(1) \\
(\underline{\mathrm{C} .6}) & \geq \tilde{d}_{l}^{2} \mu_{\varepsilon_{n}}\left(\mathscr{R}\left(x_{0}, \rho_{0}, \eta_{K}\right), 1\right)+\sum_{k=0}^{K-1} \sum_{j=1}^{N_{k}} \tilde{d}_{j, k} \mu_{\varepsilon_{n}}\left(\mathscr{R}\left(x_{0}, \eta_{k+1}, \eta_{k}\right), 1\right)-\mathcal{O}(1) \\
\text { (Prop. [46 } 3) & \geq \tilde{d}_{l} \mu_{\varepsilon_{n}}\left(\mathscr{R}\left(x_{0}, \rho_{0}, \rho\right), 1\right)+\left(\tilde{d}_{l}^{2}-\tilde{d}_{l}\right) \pi b^{2}\left|\ln \eta_{K}\right|-\mathcal{O}(1) .
\end{aligned}
$$

In the second case $\left(\operatorname{Card}\left(\Lambda_{l}\right)=1\right)$ the computations are direct

$$
\begin{aligned}
\frac{1}{2} \int_{\Omega_{l}^{n}} U_{\varepsilon_{n}}^{2}|\nabla w|^{2} & \geq \frac{1}{2} \int_{\mathscr{R}\left(x_{i}, \rho_{0}, \rho\right)} U_{\varepsilon_{n}}^{2}|\nabla w|^{2} \\
& \geq \tilde{d}_{l} \mu_{\varepsilon_{n}}\left(\mathscr{R}\left(x_{0}, \rho_{0}, \rho\right), 1\right)+\left(\tilde{d}_{l}^{2}-\tilde{d}_{l}\right) \pi b^{2}|\ln \rho|-\mathcal{O}(1) .
\end{aligned}
$$

Summing the lower bounds (D.9) and (D.10) over $l$ and applying Proposition 49, we obtain that if (D.7) occurs, then the configuration $\{\mathbf{x}, \mathbf{d}\}$ cannot be almost minimal because $\eta_{K}, \rho \rightarrow 0$ and $\tilde{d}_{l_{0}}>1$. Therefore (D.7) cannot occur for almost minimal configurations.

\section{D.4 Proof of the second part of Proposition 12}

We now prove the second part of Proposition 12, we establish the repelling effect of $\partial \Omega$ on the centers $x_{i}$ 's.

Let $x_{1}^{n}, \ldots, x_{d}^{n} \in \Omega$ and $\rho=\rho\left(\varepsilon_{n}\right) \downarrow 0$ be s.t. $\left|x_{i}^{n}-x_{j}^{n}\right| \geq 8 \rho(i \neq j)$ and $\operatorname{dist}\left(x_{1}^{n}, \partial \Omega\right) \rightarrow 0$. From the previous subsection we may assume that there is $\eta_{0}>0$ (independent of $n$ ) s.t.

$$
\min \left\{\min _{i \neq j}\left|x_{i}^{n}-x_{j}^{n}\right|, \operatorname{dist}\left(\Omega, \partial \Omega^{\prime}\right)\right\} \geq 10^{2} \eta_{0} .
$$

Up to pass to a subsequence, we may assume that $x_{i}^{n} \rightarrow a_{i} \in \bar{\Omega}$ with $a_{i} \neq a_{j}$ for $i \neq j$ and that $\eta=\max \left\{\sqrt{\operatorname{dist}\left(x_{1}^{n}, \partial \Omega\right)}, \rho\right\} \rightarrow 0$.

For sake simplicity, we assume that for $i=2, \ldots, d$ we have $a_{i} \in \Omega$. If this condition is not satisfied, then a direct adaptation of the following argument may be done. We assume that $\eta_{0}$ is s.t. for $i=2, \ldots, d$ we have $\operatorname{dist}\left(x_{i}^{n}, \partial \Omega\right) \geq 10^{2} \eta_{0}$.

We fix $x_{0}=x_{0}\left(\varepsilon_{n}\right) \in \Omega$ s.t.

$$
x_{0}-x_{1}^{n} \in \delta \mathbb{Z} \times \delta \mathbb{Z}, \operatorname{dist}\left(x_{0}^{n}, \partial \Omega\right) \geq 10^{2} \eta_{0} \text { and } \min _{i=1, \ldots, d}\left|x_{0}-x_{j}^{n}\right| \geq 10^{2} \eta_{0}
$$

We are going to prove that for $w \in H_{g}^{1}\left(\Omega^{\prime} \backslash \cup_{i} \overline{B\left(x_{i}^{n}, \rho\right)}, \mathbb{S}^{1}\right)$ we have

$$
\frac{1}{2} \int_{\mathscr{R}\left(x_{1}^{n}, \sqrt{\eta}, \eta\right)} U_{\varepsilon_{n}}^{2}|\nabla w|^{2}-\mu_{\varepsilon_{n}}\left(\mathscr{R}\left(x_{0}, \sqrt{\eta}, \eta\right), 1\right) \rightarrow \infty .
$$


Remark 54. Estimate (D.11) implies that $\left\{x_{1}^{n}, \ldots, x_{d}^{n}\right\}$ can not be an almost minimal configuration of points.

Indeed, we may construct a suitable test function $\tilde{w}$ as follows:

Construction 55. The test function $\tilde{w} \in H_{g}^{1}\left(\Omega^{\prime} \backslash\left(\overline{B\left(x_{0}, \rho\right)} \cup \cup_{i=2}^{d} \overline{B\left(x_{i}^{n}, \rho\right)}\right), \mathbb{S}^{1}\right)$

- For $i=2, \ldots, d$, we define $\tilde{w}_{\mid \mathscr{R}\left(x_{i}^{n}, \eta_{0}, \rho\right)}$ by taking a minimal map for $\frac{1}{2} \int_{\mathscr{R}\left(x_{i}^{n}, \eta_{0}, \rho\right)} U_{\varepsilon_{n}}^{2}|\nabla \cdot|^{2}$ in $H^{1}\left(\mathscr{R}\left(x_{i}^{n}, \eta_{0}, \rho\right), \mathbb{S}^{1}\right)$ with the boundary conditions $\tilde{w}\left(x_{i}^{n}+\eta_{0} \mathrm{e}^{\imath \theta}\right)=\mathrm{e}^{\imath \theta}$ and $\tilde{w}\left(x_{i}^{n}+\rho \mathrm{e}^{\imath \theta}\right)=$ $\mathrm{Cst}_{i} \mathrm{e}^{\imath \theta}, \mathrm{Cst}_{i} \in \mathbb{S}^{1}$. From Proposition 45 we have

$$
\frac{1}{2} \int_{\mathscr{R}\left(x_{i}^{n}, \eta_{0}, \rho\right)} U_{\varepsilon_{n}}^{2}|\nabla \tilde{w}|^{2} \leq \mu_{\varepsilon_{n}}\left(\mathscr{R}\left(x_{i}^{n}, \eta_{0}, \rho\right), 1\right)+C_{b}
$$

- We divide $\mathscr{R}\left(x_{0}, \eta_{0}, \rho\right)$ into $\mathscr{R}\left(x_{0}, \eta_{0}, \sqrt{\eta}\right), \mathscr{R}\left(x_{0}, \sqrt{\eta}, \eta\right)$ and $\mathscr{R}\left(x_{0}, \eta, \rho\right)$. In each of these rings we consider the minimal maps for $\frac{1}{2} \int_{\text {ring }} U_{\varepsilon_{n}}^{2}|\nabla \cdot|^{2}$ with the boundary conditions $\tilde{w}\left(x_{0}+R \mathrm{e}^{\imath \theta}\right)=\mathrm{e}^{\imath \theta}$ and $\tilde{w}\left(x_{0}+r \mathrm{e}^{\imath \theta}\right)=$ Cst $_{i} \mathrm{e}^{\imath \theta}$, Cst $_{i} \in \mathbb{S}^{1}$ where ring $\epsilon$ $\left\{\mathscr{R}\left(x_{0}, \eta_{0}, \sqrt{\eta}\right), \mathscr{R}\left(x_{0}, \sqrt{\eta}, \eta\right), \mathscr{R}\left(x_{0}, \eta, \rho\right)\right\}, r<R$ and ring $=\mathscr{R}\left(x_{0}, R, r\right)$.

Up to consider suitable rotations, we glue these functions to get a map $\tilde{w}_{\mid \mathscr{R}\left(x_{0}, \eta_{0}, \rho\right)} \in$ $H^{1}\left(\mathscr{R}\left(x_{0}, \eta_{0}, \rho\right), \mathbb{S}^{1}\right)$ which is s.t. $\tilde{w}\left(x_{0}+\eta_{0} \mathrm{e}^{\imath \theta}\right)=\mathrm{e}^{\imath \theta}$ and (from Proposition 45)

$$
\frac{1}{2} \int_{\text {ring }} U_{\varepsilon_{n}}^{2}|\nabla \tilde{w}|^{2} \leq \mu_{\varepsilon_{n}}(\operatorname{ring}, 1)+C_{b}
$$

with ring $\in\left\{\mathscr{R}\left(x_{0}, \eta_{0}, \sqrt{\eta}\right), \mathscr{R}\left(x_{0}, \sqrt{\eta}, \eta\right), \mathscr{R}\left(x_{0}, \eta, \rho\right)\right\}$.

- We extend $\tilde{w}$ in $\Omega \backslash\left(\overline{B\left(x_{0}, \eta_{0}\right)} \cup \cup_{i=2}^{d} \overline{B\left(x_{i}^{n}, \eta_{0}\right)}\right)$ using Lemma 44,1. Then we finally obtain $\tilde{w} \in H_{g}^{1}\left(\Omega^{\prime} \backslash\left(\overline{B\left(x_{0}, \rho\right)} \cup \cup_{i=2}^{d} \overline{B\left(x_{i}^{n}, \rho\right)}\right), \mathbb{S}^{1}\right)$.

From Lemma 48, (D.11) and by construction of $\tilde{w}$, for $w_{n} \in H_{g}^{1}\left(\Omega^{\prime} \backslash \cup_{i} \overline{B\left(x_{i}^{n}, \rho\right)}, \mathbb{S}^{1}\right)$ we have easily that

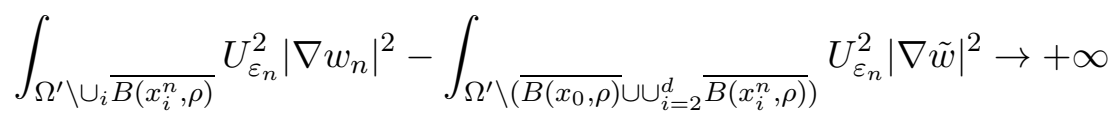

which implies that $\left\{x_{1}^{n}, \ldots, x_{d}^{n}\right\}$ can not be an almost minimal configuration of points.

We now turn to the proof of (D.11). We argue by contradiction and we assume that there is $w_{*}=w_{*}^{\varepsilon_{n}} \in H_{g}^{1}\left(\Omega^{\prime} \backslash \cup_{i} \overline{B\left(x_{i}^{n}, \rho\right)}, \mathbb{S}^{1}\right)$ s.t.

$$
\frac{1}{2} \int_{\mathscr{R}\left(x_{1}^{n}, \sqrt{\eta}, \eta\right)} U_{\varepsilon_{n}}^{2}\left|\nabla w_{*}\right|^{2} \leq \mu_{\varepsilon_{n}}\left(\mathscr{R}\left(x_{0}, \sqrt{\eta}, \eta\right), 1\right)+\mathcal{O}(1)
$$

In particular (using Lemma 48) we have

$$
\frac{1}{2} \int_{\mathscr{R}\left(x_{1}^{n}, \sqrt{\eta}, \eta\right)} U_{\varepsilon_{n}}^{2}\left|\nabla w_{*}\right|^{2}=\mu_{\varepsilon_{n}}\left(\mathscr{R}\left(x_{1}^{n}, \sqrt{\eta}, \eta\right), 1\right)+\mathcal{O}(1)
$$

The key ingredient to get a contradiction is the fact that the map $w_{*}$ is almost constant in the "half" ring $\mathscr{R}\left(x_{1}^{n}, \sqrt{\eta}, \eta\right) \backslash \Omega$.

By smoothness of $\Omega$, we may assume that the cone $K_{\sqrt{\eta}, \eta}:=\left\{x=x_{1}^{n}+\rho \mathrm{e}^{\imath \theta} \mid \theta \in\right.$ $[0, \pi / 2], \eta \leq \rho \leq \sqrt{\eta}\}$ does not intersect $\Omega: K_{\sqrt{\eta}, \eta} \cap \Omega=\emptyset$. 
We consider the map

$$
w_{0}\left(x_{1}^{n}+r \mathrm{e}^{\imath \theta}\right)=\left\{\begin{array}{ll}
\mathrm{e}^{\imath 4 \theta} & \text { if } \theta \in[0, \pi / 2] \\
1 & \text { otherwise }
\end{array}, r>0\right.
$$

which is s.t. $w_{0} \in H^{1}\left(\mathscr{R}\left(x_{1}^{n}, \sqrt{\eta}, \eta\right), \mathbb{S}^{1}\right)$ and $\operatorname{deg}_{\partial B\left(x_{1}^{n}, \sqrt{\eta}\right)}\left(w_{0}\right)=1$.

For $\tilde{d} \in \mathbb{N}^{*}$ (to be fixed later) we define the map $w_{\text {test }}=w_{*}^{\tilde{d}} w_{0} \in H^{1}\left(\mathscr{R}\left(x_{1}^{n}, \sqrt{\eta}, \eta\right), \mathbb{S}^{1}\right)$ and $\operatorname{deg}_{\partial B\left(x_{1}^{n}, \sqrt{\eta}\right)}\left(w_{\text {test }}\right)=\tilde{d}+1$.

Thus, we have

$$
\frac{1}{2} \int_{\mathscr{R}\left(x_{1}^{n}, \sqrt{\eta}, \eta\right)} U_{\varepsilon_{n}}^{2}\left|\nabla w_{\text {test }}\right|^{2} \geq \mu_{\varepsilon_{n}}\left(\mathscr{R}\left(x_{1}^{n}, \sqrt{\eta}, \eta\right), \tilde{d}+1\right)=(\tilde{d}+1)^{2} \mu_{\varepsilon_{n}}\left(\mathscr{R}\left(x_{1}^{n}, \sqrt{\eta}, \eta\right), 1\right) .
$$

On the other hand, letting $\varphi_{*}, \varphi_{0}: \mathscr{R}\left(x_{1}^{n}, \sqrt{\eta}, \eta\right) \rightarrow \mathbb{R}$ s.t. $w_{*}=\mathrm{e}^{\imath \varphi_{*}}$ and $w_{0}=\mathrm{e}^{\imath \varphi_{0}}$, (note that $\varphi_{*}, \varphi_{0}$ are locally defined and those gradients are globally defined and lie in $\left.L^{2}\left(\mathscr{R}\left(x_{1}^{n}, \sqrt{\eta}, \eta\right), \mathbb{R}\right)\right)$, we have (using $(\underline{\mathrm{D} .12})$ ),

$$
\begin{aligned}
\frac{1}{2} \int_{\mathscr{R}\left(x_{1}^{n}, \sqrt{\eta}, \eta\right)} U_{\varepsilon_{n}}^{2}\left|\nabla w_{\text {test }}\right|^{2} & =\frac{1}{2} \int_{\mathscr{R}\left(x_{1}^{n}, \sqrt{\eta}, \eta\right)} U_{\varepsilon_{n}}^{2}\left|\tilde{d} \nabla \varphi_{*}+\nabla \varphi_{0}\right|^{2} \\
= & \frac{\tilde{d}^{2}}{2} \int_{\mathscr{R}\left(x_{1}^{n}, \sqrt{\eta}, \eta\right)} U_{\varepsilon_{n}}^{2}\left|\nabla \varphi_{*}\right|^{2}+\frac{1}{2} \int_{\mathscr{R}\left(x_{1}^{n}, \sqrt{\eta}, \eta\right)} U_{\varepsilon_{n}}^{2}\left|\nabla \varphi_{0}\right|^{2}+ \\
& \quad+\tilde{d} \int_{\mathscr{R}\left(x_{1}^{n}, \sqrt{\eta}, \eta\right)} U_{\varepsilon_{n}}^{2} \nabla \varphi_{*} \cdot \nabla \varphi_{0} \\
\leq & \tilde{d}^{2} \mu_{\varepsilon_{n}}\left(\mathscr{R}\left(x_{1}^{n}, \sqrt{\eta}, \eta\right), 1\right)+2 \pi|\ln \eta|+\int_{\mathscr{R}\left(x_{1}^{n}, \sqrt{\eta}, \eta\right)} U_{\varepsilon_{n}}^{2} \nabla \varphi_{*} \cdot \nabla \varphi_{0}+\mathcal{O}(1) .
\end{aligned}
$$

Since $w_{*}=g$ in $\mathscr{R}\left(x_{1}^{n}, \sqrt{\eta}, \eta\right) \backslash \Omega$ and $\left\|\nabla \varphi_{0}\right\|_{L^{2}\left(\mathscr{R}\left(x_{1}^{n}, \sqrt{\eta}, \eta\right) \cap \Omega\right)}=0$, we have (using CauchySchwarz inequality)

$$
\int_{\mathscr{R}\left(x_{1}^{n}, \sqrt{\eta}, \eta\right)} U_{\varepsilon_{n}}^{2}\left|\nabla \varphi_{*}\right|\left|\nabla \varphi_{0}\right|=\int_{\mathscr{R}\left(x_{1}^{n}, \sqrt{\eta}, \eta\right) \backslash \bar{\Omega}} U_{\varepsilon_{n}}^{2}\left|\nabla \varphi_{*}\right|\left|\nabla \varphi_{0}\right|=\mathcal{O}(\sqrt{|\ln \eta|}) .
$$

Therefore we obtain

$$
\tilde{d}^{2} \mu_{\varepsilon_{n}}\left(\mathscr{R}\left(x_{1}^{n}, \sqrt{\eta}, \eta\right), 1\right)+2 \pi|\ln \eta|+\mathcal{O}(\sqrt{|\ln \eta|}) \geq(\tilde{d}+1)^{2} \mu_{\varepsilon_{n}}\left(\mathscr{R}\left(x_{1}^{n}, \sqrt{\eta}, \eta\right), 1\right)
$$

which implies that $2 \pi|\ln \eta|+\mathcal{O}(\sqrt{|\ln \eta|}) \geq(2 \tilde{d}+1) \mu_{\varepsilon_{n}}\left(\mathscr{R}\left(x_{1}^{n}, \sqrt{\eta}, \eta\right), 1\right) \geq(2 \tilde{d}+1) b^{2} \pi|\ln \eta|$. Clearly we obtain a contradiction taking $\tilde{d}>\left(2-b^{2}\right) /\left(2 b^{2}\right)$.

Thus, by using Remark 54, the second part of Proposition 12 is proved.

\section{D.5 Proof of the third part of Proposition 12}

In this subsection, we prove the third part of Proposition 12, the attractive effect of the inclusions.

Assume that there exist $C_{0}>0$, sequences $\varepsilon_{n}, \rho \downarrow 0, \rho=\rho\left(\varepsilon_{n}\right) \geq \varepsilon_{n}$ s.t. $\rho /(\lambda \delta) \rightarrow 0$ and distinct points $x_{1}^{n}, \ldots, x_{d}^{n}$, satisfying

$$
\inf _{\substack{w \in H_{g}^{1}\left(\Omega_{\rho}^{\prime}, \mathbb{S}^{1}\right) \\ \operatorname{deg}_{\partial B\left(x_{i}, \rho\right)}(w)=1}} \frac{1}{2} \int_{\Omega_{\rho}^{\prime}} U_{\varepsilon_{n}}^{2}|\nabla w|^{2}-I_{\rho, \varepsilon_{n}} \leq C_{0} .
$$


We denote $\mathbf{x}_{n}=\left(x_{1}^{n}, \ldots, x_{d}^{n}\right)$. From the first and the second assertion, there exists $\eta_{0}>0$ (independent of $n$ ) s.t.

$$
\min \left\{\min _{i \neq j}\left|x_{i}^{n}-x_{j}^{n}\right|, \min _{i} \operatorname{dist}\left(x_{i}^{n}, \partial \Omega\right)\right\} \geq 10^{2} \cdot \eta_{0}>0 .
$$

We want to prove that there is some $c>0$ s.t. for $i=1, \ldots, d$ we have (for large $n$ ) $B\left(x_{i}^{n}, c \lambda \delta\right) \subset \omega_{\varepsilon_{n}}$.

To this end, we argue by contradiction and we assume that either $x_{1}^{n} \notin \omega_{\varepsilon_{n}}$ or $x_{1}^{n} \in \omega_{\varepsilon_{n}}$ and $\frac{\operatorname{dist}\left(x_{1}^{n}, \partial \omega_{\varepsilon_{n}}\right)}{\lambda \delta} \rightarrow 0$.

We are going to prove that letting $y_{n} \in \delta \cdot(\mathbb{Z} \times \mathbb{Z})$ s.t. $x_{1}^{n}, y_{n} \in \overline{Y_{k, l}^{\delta}}$, then,

$$
\widehat{\mathcal{I}}_{\rho, \varepsilon_{n}}\left(\mathbf{x}_{n}, \mathbf{1}\right)-\widehat{\mathcal{I}}_{\rho, \varepsilon_{n}}\left(\left(y_{n}, x_{2}^{n}, \ldots, x_{d}^{n}\right), \mathbf{1}\right) \rightarrow \infty .
$$

Up to a subsequence, we may assume that $\lim _{n} \frac{\operatorname{dist}\left(x_{1}^{n}, \omega_{\varepsilon_{n}}\right)}{\lambda \delta}$ exists. We divide the proof into two steps:

Step 1. if $x_{1}^{n} \notin \omega_{\varepsilon_{n}}$ and $\frac{\operatorname{dist}\left(x_{1}^{n}, \omega_{\varepsilon_{n}}\right)}{\lambda \delta} \rightarrow c \in(0, \infty]$, then (D.14) holds;

Step 2. if $\frac{\operatorname{dist}\left(x_{1}^{n}, \partial \omega_{\varepsilon_{n}}\right)}{\lambda \delta} \rightarrow 0$, then (站.14 $)$ holds.

We now prove Step 1. Assume that $x_{1}^{n} \notin \omega_{\varepsilon_{n}}, \frac{\operatorname{dist}\left(x_{1}^{n}, \omega_{\varepsilon_{n}}\right)}{\lambda \delta} \rightarrow c \in(0, \infty]$ and

$$
\inf _{\substack{w \in H_{g}^{1}\left(\Omega_{\rho}^{\prime}, \mathbb{S}^{1}\right) \\ \operatorname{deg}_{\partial B\left(x_{i}, \rho\right)}(w)=1}} \frac{1}{2} \int_{\Omega_{\rho}^{\prime}} U_{\varepsilon_{n}}^{2}|\nabla w|^{2}-I_{\rho, \varepsilon_{n}} \leq C_{0}
$$

Denote $w_{n}$ a minimizer for $\widehat{\mathcal{I}}_{\rho, \varepsilon_{n}}\left(\mathbf{x}_{n}, \mathbf{1}\right)$ (see Proposition 8). Using Lemma 52, 2 , for $\rho \leq$ $r<R<\eta_{0}$, one has

$$
\frac{1}{2} \int_{B\left(x_{1}^{n}, R\right) \backslash \overline{B\left(x_{1}^{n}, r\right)}} U_{\varepsilon_{n}}^{2}\left|\nabla w_{n}\right|^{2}-\mu_{\varepsilon_{n}}\left(B\left(x_{1}^{n}, R\right) \backslash \overline{B\left(x_{1}^{n}, r\right)}, 1\right) \leq C_{0}+C\left(\eta_{0}\right) .
$$

Let $\kappa \in\left(0,10^{-2} \cdot c\right)$ be s.t. $B\left(0,10^{2} \kappa\right) \subset \omega \subset Y$ and $\operatorname{dist}(\omega, \partial Y) \geq 10^{2} \kappa$.

From Lemma 52 (Assertions 2 and 3), we have

$$
\widehat{\mathcal{I}}_{\rho, \varepsilon_{n}}\left(\mathbf{x}_{n}, \mathbf{1}\right)=\sum_{i=1}^{d} \mu_{\varepsilon_{n}}\left(\mathscr{R}\left(x_{i}^{n}, \eta_{0}, \rho\right), 1\right)+\mathcal{O}(1)
$$

and

$$
\widehat{\mathcal{I}}_{\rho, \varepsilon_{n}}\left(\left(y_{n}, x_{2}^{n}, \ldots, x_{d}^{n}\right), \mathbf{1}\right)=\mu_{\varepsilon_{n}}\left(\mathscr{R}\left(y_{n}, \eta_{0}, \rho\right), 1\right)+\sum_{i=2}^{d} \mu_{\varepsilon_{n}}\left(\mathscr{R}\left(x_{i}^{n}, \eta_{0}, \rho\right), 1\right)+\mathcal{O}(1) .
$$

Recall that $y_{n} \in \delta \cdot \mathbb{Z}^{2}$ is s.t. $x_{1}^{n}, y_{n} \in \overline{Y_{k, l}^{\delta}}$. Since $\left|x_{1}^{n}-y_{n}\right| \leq \delta$, using Lemma 44,2 and Propositions 45, 46,3, we have

$$
\mu_{\varepsilon_{n}}\left(\mathscr{R}\left(y_{n}, \eta_{0}, \rho\right), 1\right)=\mu_{\varepsilon_{n}}\left(\mathscr{R}\left(x_{1}^{n}, \eta_{0}, \delta\right), 1\right)+\mu_{\varepsilon_{n}}\left(\mathscr{R}\left(y_{n}, \kappa \delta, \rho\right), 1\right)+\mathcal{O}(1)
$$


Therefore

$$
\begin{aligned}
& \widehat{\mathcal{I}}_{\rho, \varepsilon_{n}}\left(\mathbf{x}_{n}, \mathbf{1}\right)-\widehat{\mathcal{I}}_{\rho, \varepsilon_{n}}\left(\left(y_{n}, x_{2}^{n}, \ldots, x_{d}^{n}\right), \mathbf{1}\right) \\
& \quad=\mu_{\varepsilon_{n}}\left(\mathscr{R}\left(x_{1}^{n}, \kappa \delta, \rho\right), 1\right)-\mu_{\varepsilon_{n}}\left(\mathscr{R}\left(y_{n}, \kappa \delta, \rho\right), 1\right)+\mathcal{O}(1) .
\end{aligned}
$$

Thus it suffices to estimate the energies in the rings with radii $\kappa \delta$ and $\rho$. We have (using (1.5))

$$
\mu_{\varepsilon_{n}}\left(\mathscr{R}\left(y_{n}, \kappa \delta, \rho\right), 1\right)=\pi|\ln \lambda|+b^{2} \pi \ln \frac{\lambda \delta}{\rho}+\mathcal{O}(1) .
$$

In order to estimate $\mu_{\varepsilon_{n}}\left(\mathscr{R}\left(x_{1}^{n}, \kappa \delta, \rho\right), 1\right)$, we divide the argument according to the asymptotic of $\lambda$. If $\lambda \equiv 1$, then $c \in(0, \infty)$ and thus $\operatorname{dist}\left(B\left(x_{i}, c \delta / 3, \omega_{\varepsilon_{n}}\right) \geq c \delta / 3\right.$. Consequently, from Proposition 3, we have

$$
\mu_{\varepsilon_{n}}\left(\mathscr{R}\left(x_{1}^{n}, \kappa \delta, \rho\right), 1\right)=\pi \ln \frac{\delta}{\rho}+\mathcal{O}(1)
$$

Therefore (D.14) holds.

If $\lambda \rightarrow 0$, we let $\chi=\left\{\begin{array}{ll}\frac{c}{2} \lambda \delta & \text { if } c<\infty \\ \kappa \lambda \delta & \text { otherwise }\end{array}\right.$ and $\eta=\operatorname{dist}\left(x_{1}^{n}, \partial \omega_{\varepsilon_{n}}\right)$. Note that $\frac{\eta+2 \lambda \delta}{\eta-\chi}=$ $\mathcal{O}(1)$ and that $U_{\varepsilon_{n}}=1+V_{n}$ in $\mathscr{R}\left(x_{1}^{n}, \kappa \delta, \eta+2 \lambda \delta\right) \cup \mathscr{R}\left(x_{1}^{n}, \eta-\chi, \rho\right),\left\|V_{n}\right\|_{L^{\infty}}=o\left(\varepsilon_{n}^{2}\right)$ (from $(1.5)$.

Thus we obtain

$$
\begin{aligned}
\mu_{\varepsilon_{n}}\left(\mathscr{R}\left(x_{1}^{n}, \kappa \delta, \rho\right), 1\right) & \geq \pi \ln \frac{\delta}{\eta+2 \lambda \delta}+\pi b^{2} \ln \frac{\eta+2 \lambda \delta}{\eta-\chi}+\pi \ln \frac{\eta-\chi}{\rho}+\mathcal{O}(1) \\
& =\pi \ln \frac{\delta}{\rho}+\mathcal{O}(1) .
\end{aligned}
$$

Therefore if $c \in(0, \infty]$, then (D.17) holds. Estimates (D.15), (D.16) and (D.17) contradict (D.13) (because (D.14) holds).

We now turn to Step 2. Arguing as in Step 1., it suffices to prove that

$$
\left.\mu_{\varepsilon_{n}}\left(\mathscr{R}\left(x_{1}^{n}, \kappa \delta, \rho\right), 1\right)-\mu_{\varepsilon_{n}}\left(\mathscr{R}\left(y_{n}, \kappa \delta, \rho\right), 1\right), 1\right) \rightarrow \infty \text { for some fixed } \kappa .
$$

(And $y_{n} \in \delta \cdot \mathbb{Z}^{2}$ s.t. $x_{i}^{n}, y_{n} \in \overline{Y_{k, l}^{\delta}}$ )

We let $\kappa>0$ (depending only on $\omega$ ) be s.t.

$$
\kappa<10^{-2} \cdot \operatorname{dist}(\omega, \partial Y) \text { and } B\left(0,10^{2} \cdot \kappa\right) \subset \omega .
$$

In order to prove (D.18), we divide the annular $\mathscr{R}\left(x_{1}^{n}, \kappa \delta, \rho\right)$ into three regions :

$$
\mathscr{R}\left(y_{n}, \kappa \delta, \rho\right)=\mathscr{R}\left(x_{1}^{n}, \kappa \delta, \kappa \lambda \delta\right) \cup \overline{\mathscr{R}\left(x_{1}^{n}, \kappa \lambda \delta, r_{n}\right)} \cup \mathscr{R}\left(x_{1}^{n}, r_{n}, \rho\right)
$$

with

$$
r_{n}=\max \left\{\varepsilon_{n}^{1 / 4}, \rho, \sqrt{\lambda \delta \cdot \operatorname{dist}\left(x_{n}, \partial \omega_{\varepsilon}\right)}\right\}+\sqrt{\varepsilon_{n}} .
$$

We are going to prove that $\mu_{\varepsilon_{n}}\left(\mathscr{R}\left(x_{1}^{n}, \kappa \lambda \delta, r_{n}\right), 1\right)$ is too large.

We consider $K_{n}$ the cone of vertex $x_{1}^{n}$ and aperture $\pi / 2$ which admits the line $\left(x_{1}^{n}, \Pi_{\partial \omega_{\varepsilon_{n}}} x_{1}^{n}\right)$ for symmetry axis and s.t. $K_{n} \cap \omega_{\varepsilon_{n}} \cap \mathscr{R}\left(x_{1}^{n}, \kappa \lambda \delta, r_{n}\right)=\emptyset$. Here $\Pi_{\partial \omega_{\varepsilon_{n}}}\left(x_{1}^{n}\right)$ is the orthogonal projection of $x_{1}^{n}$ on $\partial \omega_{\varepsilon_{n}}$. 


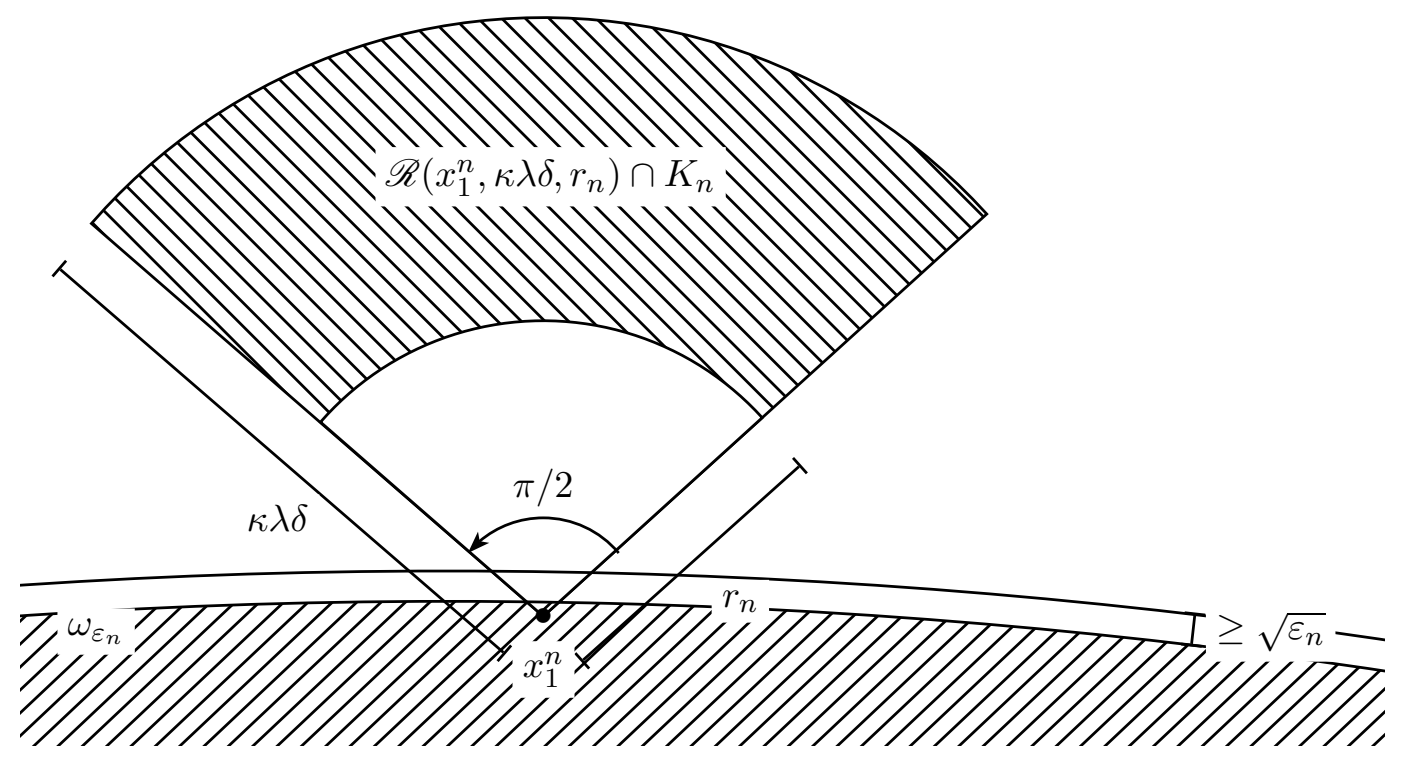

Figure 6: The domain $\mathscr{R}\left(x_{1}^{n}, \kappa \lambda \delta, r_{n}\right) \cap K_{n}$

Note that since $\frac{\operatorname{dist}\left(x_{1}^{n}, \omega_{\varepsilon_{n}}\right)}{\lambda \delta} \rightarrow 0$, for large $n$ and small $\kappa$ (independently of $n$ ), by smoothness of $\omega, K_{n}$ is well defined (see Figure 6 ).

We have $U_{\varepsilon_{n}}=1+V_{n}$ in $\mathscr{R}\left(x_{1}^{n}, \kappa \lambda \delta, r_{n}\right) \cap K_{n}$ where, $\left\|V_{n}\right\|_{L^{\infty}}=o\left(\varepsilon_{n}^{2}\right)$. Thus, if we define $\alpha_{n}=\left\{\begin{array}{ll}1 & \text { in } K_{n} \\ b^{2} & \text { otherwise }\end{array}\right.$, then, from Lemma 53 with $\theta_{0}=3 \pi / 2$, for $w \in$ $H^{1}\left(\mathscr{R}\left(x_{1}^{n}, \kappa \lambda \delta, r_{n}\right), \mathbb{S}^{1}\right)$ s.t. $\operatorname{deg}_{\partial B\left(x_{1}^{n}, r_{n}\right)}(w)=1$, we have

$$
\frac{1}{2} \int_{\mathscr{R}\left(x_{1}^{n}, \kappa \lambda \delta, r_{n}\right)} \alpha_{n}|\nabla w|^{2} \geq b^{2} \frac{4 \pi}{b^{2}+3} \ln \frac{\kappa \lambda \delta}{r_{n}} .
$$

Clearly, from construction, $U_{\varepsilon_{n}}^{2} \geq \alpha_{n}+o\left(\varepsilon_{n}^{2}\right)$, thus if $w_{n}$ is a minimal map for $\widehat{\mathcal{I}}_{\rho, \varepsilon_{n}}\left(\mathbf{x}_{n}, \mathbf{1}\right)$, then we have

$$
\frac{1}{2} \int_{\mathscr{R}\left(x_{1}^{n}, \kappa \lambda \delta, r_{n}\right)} U_{\varepsilon_{n}}^{2}\left|\nabla w_{n}\right|^{2} \geq b^{2} \frac{4 \pi}{b^{2}+3} \ln \frac{\kappa \lambda \delta}{r_{n}}+o_{n}(1) .
$$

Now the computations are direct

$$
\begin{aligned}
\mu_{\varepsilon_{n}}\left(\mathscr{R}\left(x_{1}^{n}, \kappa \delta, \rho\right), 1\right)= & \mu_{\varepsilon_{n}}\left(\mathscr{R}\left(x_{1}^{n}, \kappa \delta, 3 \lambda \delta\right), 1\right)+\mu_{\varepsilon_{n}}\left(\mathscr{R}\left(x_{1}^{n}, \kappa \lambda \delta, r_{n}\right), 1\right) \\
& \quad+\mu_{\varepsilon_{n}}\left(\mathscr{R}\left(x_{1}^{n}, r_{n}, \rho\right), 1\right)+\mathcal{O}(1) \\
\geq & \pi|\ln \lambda|+b^{2} \frac{4 \pi}{b^{2}+3} \ln \frac{\lambda \delta}{r_{n}}+b^{2} \pi \ln \frac{r_{n}}{\rho}+\mathcal{O}(1) .
\end{aligned}
$$

Therefore, (D.18) is a direct consequence of (D.16) and (D.19) since $\frac{\lambda \delta}{r_{n}} \rightarrow+\infty$.

\section{E Proof of Propositions 16 and 17}

We now prove the results specific to th pinning terms with dilution. We begin these proofs by their key ingredient. 


\section{E.1 An important effect of the dilution of inclusions}

We first state a result which establishes that a "sufficiently large" circle has a small intersection with $\omega_{\varepsilon}$ if $\lambda \rightarrow 0$.

Lemma 56. We denote $C_{\rho}$ a circle with radius $\rho$.

1. Assume that the pinning term is periodic. Once $\lambda \leq 1 / 8 \pi$, for $\rho \geq \delta / 3$ we have $\mathscr{H}^{1}\left(C_{\rho} \cap \omega_{\varepsilon}\right) \leq 16 \pi^{2} \lambda \rho$.

2. Assume that the pinning term is not periodic, and recall that the inclusions with size $\lambda \delta^{j}$ is $\omega_{\varepsilon}^{j}=\cup_{i \in \mathcal{M}_{j}^{\varepsilon}}\left\{y_{i, j}^{\varepsilon}+\lambda \delta^{j} \cdot \omega\right\}, j \in\{1, \ldots, P\}$.

Once $\lambda \leq 1 / 8 \pi$, for $\rho \geq \delta^{j} / 3$ we have $\mathscr{H}^{1}\left(C_{\rho} \cap \omega_{\varepsilon}^{j}\right) \leq 16 \pi^{2} \lambda \rho$.

Here $\mathscr{H}^{1}\left(C_{\rho} \cap \omega_{\varepsilon}\right)$ is the 1-dimensional Hausdorff measure of $C_{\rho} \cap \omega_{\varepsilon}$.

Proof. In order to unify the notations, we fix $j=1$ if we are in the periodic case (and $j \in\left\{1, \ldots, P \mid M_{j}^{\varepsilon} \in \mathbb{N}^{*}\right\}$ if we are in the non-periodic case).

Assume that $C_{\rho} \cap \omega_{\varepsilon} \neq \emptyset$ and let

$$
S_{j}:=\left\{\begin{array}{l|l}
\left\{\tilde{Y}_{\varepsilon}=(\delta k, \delta l)+\delta \cdot Y\right. & \begin{array}{c}
k, l \in \mathbb{Z}^{2}, \tilde{Y}_{\varepsilon} \subset \Omega \\
\text { and } \tilde{Y}_{\varepsilon} \cap C_{\rho} \neq \emptyset
\end{array}
\end{array}\right\} \quad \text { in the periodic case }
$$

For $\tilde{Y}_{\varepsilon} \in S_{j}$, we denote

- $\tilde{\omega}_{\varepsilon}$ the connected component of $\omega_{\varepsilon}$ which is included in $\tilde{Y}_{\varepsilon}$

- $z_{j}$ the center of $\tilde{Y}_{\varepsilon}=\left\{\begin{array}{ll}z_{1}+\delta \cdot Y, z_{1} \in \delta \cdot \mathbb{Z}^{2} & \text { in the periodic case } \\ B\left(z_{j}, \delta^{j}\right), z_{j} \in \mathcal{M}_{j}^{\varepsilon} & \text { in the non-periodic case }\end{array}\right.$.

We first treat the case where $C_{\rho} \subset \tilde{Y}_{\varepsilon} \in S_{j}$ : since $\rho \geq \delta^{j} / 3$ and $\tilde{\omega}_{\varepsilon} \subset B\left(z_{j}, \lambda \delta^{j}\right)$ (because $\omega \subset B(0,1)$ ), we have

$$
\mathscr{H}^{1}\left(C_{\rho} \cap \tilde{\omega}_{\varepsilon}\right)=\mathscr{H}^{1}\left(C_{\rho} \cap \omega_{\varepsilon}^{j}\right) \leq \mathscr{H}^{1}\left(\partial B\left(z_{j}, \lambda \delta^{j}\right)\right)=2 \pi \lambda \delta^{j} \leq 6 \pi \lambda \rho .
$$

Otherwise, for $\tilde{Y}_{\varepsilon} \in S_{j}, C_{\rho} \nsubseteq \tilde{Y}_{\varepsilon}$ and thus

$$
\left.\mathscr{H}^{1}\left(C_{\rho} \cap \tilde{\omega}_{\varepsilon}\right) \leq \mathscr{H}^{1}\left(C_{\rho} \cap \overline{B\left(y_{i, j}^{\varepsilon}, \lambda \delta^{j}\right)}\right) \leq 2 \pi \lambda \delta^{j} \text { (because } \omega \subset B(0,1)\right)
$$

and

$$
\mathscr{H}^{1}\left(C_{\rho} \cap \tilde{Y}_{\varepsilon} \backslash \tilde{\omega}_{\varepsilon}\right) \geq \delta^{j} \cdot\left(\frac{1}{2}-2 \pi \lambda\right) .
$$

Last estimate comes from the fact that $C_{\rho} \nsubseteq \tilde{Y}_{\varepsilon}$. Thus $\mathscr{H}^{1}\left(C_{\rho} \cap \tilde{Y}_{\varepsilon} \backslash \tilde{\omega}_{\varepsilon}\right)$ is at least a radius of $\tilde{Y}_{\varepsilon}$ (if we are in the non periodic or half of side length of $\tilde{Y}_{\varepsilon}$ otherwise) minus the previous upper bound. Thus we obtain (for $\lambda \leq 1 / 8 \pi$ )

$$
\mathscr{H}^{1}\left(C_{\rho} \cap \tilde{\omega}_{\varepsilon}\right) \leq 2 \pi \lambda \frac{\mathscr{H}^{1}\left(C_{\rho} \cap \tilde{Y}_{\varepsilon} \backslash \tilde{\omega}_{\varepsilon}\right)}{\frac{1}{2}-2 \pi \lambda} \leq 8 \pi \lambda \mathscr{H}^{1}\left(C_{\rho} \cap \tilde{Y}_{\varepsilon} \backslash \tilde{\omega}_{\varepsilon}\right) .
$$


Consequently,

$$
\begin{aligned}
\mathscr{H}^{1}\left(C_{\rho} \cap \omega_{\varepsilon}\right) & =\sum_{\tilde{Y}_{\varepsilon} \in S_{j}} \mathscr{H}^{1}\left(C_{\rho} \cap \tilde{\omega}_{\varepsilon}\right) \\
& \leq 8 \pi \lambda \sum_{\tilde{Y}_{\varepsilon} \in S_{j}} \mathscr{H}^{1}\left(C_{\rho} \cap \tilde{Y}_{\varepsilon} \backslash \tilde{\omega}_{\varepsilon}\right) \leq 8 \pi \lambda \mathscr{H}^{1}\left(C_{\rho}\right)=16 \pi^{2} \lambda \rho .
\end{aligned}
$$

\section{E.2 Proof of Proposition 16}

We are now in position to prove Proposition 16. The proof is done in 3 steps.

Let $\varepsilon_{n} \downarrow 0, \rho=\rho\left(\varepsilon_{n}\right) \downarrow 0, \rho \geq \varepsilon_{n}$ and let $\mathbf{x}_{n}$ be a quasi-minimizer for $J_{\rho, \varepsilon_{n}}$ (defined Notation [15).

From Corollaries 13 \& 14, up to pass to a subsequence, there are $\eta_{0}>0$ and $\mathbf{a}=$ $\left(a_{1}, \ldots, a_{d}\right) \in \Omega^{d}$ s.t. $x_{i}^{n} \rightarrow a_{i},\left|a_{i}-a_{j}\right|$, $\operatorname{dist}\left(a_{i}, \partial \Omega\right)>10^{2} \eta_{0}$.

We prove that $W_{g}\left(a_{1}, \ldots, a_{d}\right)=\min _{b_{1}, \ldots, b_{n} \in \Omega} W_{g}\left(b_{1}, \ldots, b_{n}\right)$. We argue by contradiction and we assume that, up to consider a smaller value for $\eta_{0}$ if necessary, we have the existence of $\mathbf{b}=\left(b_{1}, \ldots, b_{d}\right) \in \Omega^{d}$ s.t. $\left|b_{i}-b_{j}\right| \geq 10^{2} \eta_{0}, \operatorname{dist}\left(b_{i}, \partial \Omega\right)>10^{2} \eta_{0}$ and

$$
W_{g}(\mathbf{b})<W_{g}(\mathbf{a})-10^{2} \eta_{0} .
$$

Step 1. We estimate the energies in perforated domains with a fixed perforation size

The goal of this step is to prove the existence of small $\rho_{0}$ (independent of $n$ ) s.t. we have for $\mathbf{c} \in\{\mathbf{a}, \mathbf{b}\}$ and $\mathbf{x} \in \Omega^{d}$ satisfying $\max _{i}\left|x_{i}-c_{i}\right| \leq \rho_{0}$

$$
\hat{\mathcal{J}}_{\rho_{0}, \mathbb{I}}(\mathbf{x})-\hat{\mathcal{J}}_{\rho_{0}, \varepsilon_{n}}(\mathbf{x}) \leq 2 \eta_{0} .
$$

From [10] ((15) and Lemma 2), we may fix $\eta_{0}>\rho_{0}>0$ independent of $n$ s.t. for $\mathbf{c} \in\{\mathbf{a}, \mathbf{b}\}$, we have

$$
\begin{gathered}
\hat{\mathcal{J}}_{\rho_{0}, \mathbb{I}}(\mathbf{x})-\hat{\mathcal{I}}_{\rho_{0}, \mathbb{I}}(\mathbf{x}) \leq \eta_{0} \text { for all } \mathbf{x} \in \Omega^{d} \text { s.t. } \max _{i}\left|x_{i}-c_{i}\right| \leq \rho_{0}, \\
\left|\hat{\mathcal{I}}_{\rho_{0}, \mathbb{I}}(\mathbf{x})-\pi d\right| \ln \rho_{0}\left|-W_{g}(\mathbf{x})\right| \leq \eta_{0} \text { for all } \mathbf{x} \in \Omega^{d} \text { s.t. } \max _{i}\left|x_{i}-c_{i}\right| \leq \rho_{0}
\end{gathered}
$$

and

$$
\left|W_{g}(\mathbf{c})-W_{g}(\mathbf{x})\right| \leq \eta_{0} \text { for all } \mathbf{x} \in \Omega^{d} \text { s.t. } \max _{i}\left|x_{i}-c_{i}\right| \leq \rho_{0} .
$$

For $\mathbf{c} \in\{\mathbf{a}, \mathbf{b}\}$ and $\mathbf{x} \in \Omega^{d}$ s.t. $\max _{i}\left|x_{i}-c_{i}\right| \leq \rho_{0}$ :

- We let $\theta_{\mathbf{x}}=\sum_{i=1}^{d} \theta_{x_{i}}$ where $\theta_{x_{i}} \in(-\pi, \pi], \frac{x-x_{i}}{\left|x-x_{i}\right|}=\mathrm{e}^{\imath \theta_{x_{i}}}\left(x \neq x_{i}\right)$ is main determination of the argument of $x-x_{i}$.

- We fix $\phi_{0}^{\mathbf{x}} \in C^{\infty}(\partial \Omega, \mathbb{R})$ s.t. $\mathrm{e}^{\imath \phi_{0}^{\mathbf{x}}}=g \mathrm{e}^{-\imath \theta_{\mathbf{x}}}$. Clearly, since $\operatorname{deg}_{\partial \Omega}\left(g \mathrm{e}^{-\imath \theta_{\mathbf{x}}}\right)=0$, and since $g \mathrm{e}^{-\imath \theta_{\mathbf{x}}} \in C^{\infty}\left(\partial \Omega, \mathbb{S}^{1}\right), \phi_{0}^{\mathbf{x}} \in C^{\infty}(\partial \Omega, \mathbb{R})$ is well defined [3].

- We let $\phi_{*}=\phi_{*}^{\mathbf{x}}, \phi=\phi^{\mathbf{x}} \in H^{1}$ be the solutions of

$$
\begin{cases}-\Delta \phi_{*}=0 & \text { in } \Omega \backslash \cup \overline{\left(x_{i}, \rho_{0}\right)} \\ \phi_{*}=\phi_{0} & \text { on } \partial \Omega \\ \partial_{\nu} \phi_{*}=-\sum_{j \neq i} \partial_{\nu} \theta_{x_{j}} & \text { on } \partial B\left(x_{i}, \rho_{0}\right), i=1, \ldots, d\end{cases}
$$


and

$$
\left\{\begin{array}{ll}
-\operatorname{div}\left(U_{\varepsilon}^{2} \nabla \phi\right)=\operatorname{div}\left(U_{\varepsilon}^{2} \nabla \theta_{\mathbf{x}}\right) & \text { in } \Omega \backslash \cup \overline{B\left(x_{i}, \rho_{0}\right)} \\
\phi=\phi_{0} & \text { on } \partial \Omega \\
\partial_{\nu} \phi=-\sum_{j \neq i} \partial_{\nu} \theta_{x_{j}} & \text { on } \partial B\left(x_{i}, \rho_{0}\right), i=1, \ldots, d
\end{array} .\right.
$$

- We let $\psi=\phi-\phi_{*}$ be the solution of

$$
\left\{\begin{array}{ll}
-\operatorname{div}\left(U_{\varepsilon}^{2} \nabla \psi\right)=\operatorname{div}\left[\left(U_{\varepsilon}^{2}-1\right)\left(\nabla \theta_{\mathbf{x}}-\nabla \phi_{*}\right)\right] & \text { in } \Omega \backslash \cup \overline{B\left(x_{i}, \rho_{0}\right)} \\
\psi=0 & \text { on } \partial \Omega \\
\partial_{\nu} \psi=0 & \text { on } \partial B\left(x_{i}, \rho_{0}\right), i=1, \ldots, d
\end{array} .\right.
$$

Remark 57. 1. From Proposition 8, the functions $\phi_{*}, \phi$ are s.t. $w_{*}=\mathrm{e}^{\imath\left(\theta_{\mathbf{x}}+\phi_{*}\right)}, w=$ $\mathrm{e}^{\imath\left(\theta_{\mathbf{x}}+\phi_{*}\right)} \in \mathcal{I}_{\rho_{0}}(\mathbf{x})$ satisfy

$$
\hat{\mathcal{I}}_{\rho_{0}, \mathbb{I}}(\mathbf{x})=\frac{1}{2} \int_{\Omega \backslash \cup \overline{B\left(x_{i}, \rho_{0}\right)}}\left|\nabla w_{*}\right|^{2}=\frac{1}{2} \int_{\Omega \backslash \cup \overline{B\left(x_{i}, \rho_{0}\right)}}\left|\nabla\left(\theta_{\mathbf{x}}+\phi_{*}\right)\right|^{2}
$$

and

$$
\hat{\mathcal{I}}_{\rho_{0}, \varepsilon}(\mathbf{x})=\frac{1}{2} \int_{\Omega \backslash \cup \overline{B\left(x_{i}, \rho_{0}\right)}} U_{\varepsilon}^{2}|\nabla w|^{2}=\frac{1}{2} \int_{\Omega \backslash \cup \overline{B\left(x_{i}, \rho_{0}\right)}} U_{\varepsilon}^{2}\left|\nabla\left(\theta_{\mathbf{x}}+\phi\right)\right|^{2} .
$$

2. $\nabla \phi$ and $\nabla \phi_{*}$ are bounded independently of $\mathbf{x}$ and $\varepsilon_{n}$ in $L^{2}\left(\Omega \backslash \cup \overline{B\left(x_{i}, \rho_{0}\right)}\right)$.

3. From a Poincaré inequality we have the existence of $C_{0}$ independent of $\mathbf{x}$ s.t.

$$
\|\psi\|_{L^{2}\left(\Omega \backslash \cup \overline{B\left(x_{i}, \rho_{0}\right)}\right)} \leq C_{0}\|\nabla \psi\|_{L^{2}\left(\Omega \backslash \cup \overline{B\left(x_{i}, \rho_{0}\right)}\right)} .
$$

Therefore, using a trace inequality in $\mathscr{R}\left(x_{i}, 2 \rho_{0}, \rho_{0}\right)$ we obtain $\|\psi\|_{L^{2}\left(\partial B\left(x_{i}, \rho_{0}\right)\right)} \leq C_{0}^{\prime}$, $C_{0}^{\prime}$ is independent of $\mathbf{x}, n$.

4. We have $\left|\nabla \phi_{*}\right|$ which is bounded in $L^{\infty}\left(\Omega \backslash \cup \overline{B\left(x_{i}, \rho_{0}\right)}\right)$ :

$$
\left|\nabla \phi_{*}\right| \leq C_{0} \text { with } C_{0} \text { independent of } \mathbf{x} \text {. }
$$

Indeed, with standard result of elliptic interior regularity, we have

$$
\left\|\phi_{*}\right\|_{C^{2}\left(\partial B\left(x_{i}, 8 \rho_{0}\right)\right)},\left\|\phi_{*}\right\|_{C^{2}\left(\partial B\left(c_{i}, 4 \rho_{0}\right)\right)} \leq C_{0}^{\prime} .
$$

Thus, from global regularity for the Laplacian, we have

$$
\left\|\nabla \phi_{*}\right\|_{L^{\infty}\left(\Omega \backslash \cup \overline{B\left(c_{i}, 4 \rho_{0}\right)}\right)},\left\|\nabla \phi_{*}\right\|_{L^{\infty}\left(\mathscr{R}\left(x_{i}, 8 \rho_{0}, \rho_{0}\right)\right)} \leq C_{0}^{\prime \prime} .
$$

We let $\Omega_{\rho_{0}}=\Omega_{\rho_{0}}(\mathbf{x}):=\Omega \backslash \cup \overline{B\left(x_{i}, \rho_{0}\right)}$. We are now in position to prove that $\int_{\Omega_{\rho_{0}}}|\nabla \psi|^{2} \rightarrow$ 0 when $n \rightarrow \infty$ uniformly on $\mathbf{x}$. This estimate will easily imply (E.1). Indeed

$$
\begin{aligned}
& \hat{\mathcal{I}}_{\rho_{0}, \mathbb{I}}(\mathbf{x})-\hat{\mathcal{I}}_{\rho_{0}, \varepsilon_{n}}(\mathbf{x})= \frac{1}{2} \int_{\Omega_{\rho_{0}}} U_{\varepsilon_{n}}^{2}\left[\left|\nabla\left(\theta_{\mathbf{x}}+\phi_{*}\right)\right|^{2}-\left|\nabla\left(\theta_{\mathbf{x}}+\phi\right)\right|^{2}\right] \\
&+\frac{1}{2} \int_{\Omega_{\rho_{0}}}\left(1-U_{\varepsilon_{n}}^{2}\right)\left|\nabla\left(\theta_{\mathbf{x}}+\phi_{*}\right)\right|^{2} \\
&\left(\begin{array}{c}
\text { Cauchy-Schwarz } \\
\text { inequality }
\end{array}\right) \leq \tilde{C}_{0}\left(\|\nabla \psi\|_{L^{2}\left(\Omega_{\rho_{0}}\right)}+\left\|1-U_{\varepsilon_{n}}^{2}\right\|_{L^{2}\left(\Omega_{\rho_{0}}\right)}\right) \rightarrow 0 .
\end{aligned}
$$


Consequently we obtain

$$
\hat{\mathcal{J}}_{\rho_{0}, \mathbb{I}}(\mathbf{x})-\hat{\mathcal{J}}_{\rho_{0}, \varepsilon_{n}}(\mathbf{x}) \leq \hat{\mathcal{I}}_{\rho_{0}, \mathbb{I}}(\mathbf{x})-\hat{\mathcal{I}}_{\rho_{0}, \varepsilon_{n}}(\mathbf{x})+\eta_{0} \leq \eta_{0}+o_{n}(1) \leq 2 \eta_{0}
$$

which is exactly (E.1).

Thus it remains to establish that $\int_{\Omega_{\rho_{0}}}|\nabla \psi|^{2} \rightarrow 0$ when $n \rightarrow \infty$ uniformly on $\mathbf{x}$ :

$$
\begin{aligned}
\int_{\Omega_{\rho_{0}}} U_{\varepsilon_{n}}^{2}|\nabla \psi|^{2} & =\int_{\Omega_{\rho_{0}}} \operatorname{div}\left[\left(U_{\varepsilon_{n}}^{2}-1\right)\left(\nabla \theta_{\mathbf{x}}-\nabla \phi_{*}\right)\right] \psi \\
& =\int_{\Omega_{\rho_{0}}}\left(1-U_{\varepsilon_{n}}^{2}\right)\left(\nabla \theta_{\mathbf{x}}-\nabla \phi_{*}\right) \cdot \nabla \psi+\int_{\partial \Omega_{\rho_{0}}}\left(U_{\varepsilon_{n}}^{2}-1\right) \partial_{\nu}\left(\theta_{\mathbf{x}}-\phi_{*}\right) \psi
\end{aligned}
$$

From the $L^{2}$ bound on $\nabla \psi$ and the $L^{\infty}$ bounds on $\nabla \phi_{*}, \nabla \theta_{\mathbf{x}}$ we have (with $C_{0}$ independent of $\mathbf{x}$ )

$$
\begin{aligned}
\int_{\Omega_{\rho_{0}}} U_{\varepsilon_{n}}^{2}|\nabla \psi|^{2} & \leq\left(\int_{\Omega_{\rho_{0}}}\left|1-U_{\varepsilon_{n}}^{2}\right|^{2}\left|\nabla \theta_{\mathbf{x}}-\nabla \phi_{*}\right|^{2}\right)^{1 / 2}\left(\int_{\Omega_{\rho_{0}}}|\nabla \psi|^{2}\right)^{1 / 2}+ \\
& +\left(\int_{\partial \Omega_{\rho_{0}}}\left(U_{\varepsilon_{n}}^{2}-1\right)^{2}\left|\partial_{\nu}\left(\theta_{\mathbf{x}}-\phi_{*}\right)\right|^{2}\right)^{1 / 2}\left(\int_{\partial \Omega_{\rho_{0}}}|\psi|^{2}\right)^{1 / 2} \\
& \leq C_{0}\left(\left\|1-U_{\varepsilon_{n}}^{2}\right\|_{L^{2}\left(\Omega_{\rho_{0}}\right)}+\left\|1-U_{\varepsilon_{n}}^{2}\right\|_{L^{2}\left(\partial \Omega_{\rho_{0}}\right)}\right) .
\end{aligned}
$$

From Proposition 3 and Lemma 56 we have $\left\|1-U_{\varepsilon_{n}}^{2}\right\|_{L^{2}\left(\partial \Omega_{\rho_{0}}\right)}=\mathcal{O}(\lambda)$ uniformly in $\mathbf{x}$.

Therefore $\int_{\Omega \backslash \cup \overline{B\left(x_{i}, \rho_{0}\right)}}|\nabla \psi|^{2} \rightarrow 0$ when $n \rightarrow \infty$ uniformly on $\mathbf{x}$ and (E.1) holds.

Step 2. We study the energies in $\mathscr{R}\left(x_{i}, \rho_{0}, \max \left(\delta, \lambda^{2}\right)\right)$

Let

- $\kappa=\max (\lambda, \sqrt{\delta})$

- $\mathbf{x}_{n}$ be a quasi minimizer for $J_{\rho, \varepsilon}$

- $w_{n}=\mathrm{e}^{\imath \varphi_{n}}$ be a minimizer of $\hat{\mathcal{J}}_{\rho, \varepsilon_{n}}\left(\mathbf{x}_{n}\right)\left(\varphi_{n}\right.$ is locally defined and its gradient is globally defined in $\left.\Omega \backslash \cup \overline{B\left(x_{i}, \rho\right)}\right)$.

We prove that there is $r \in\left(\kappa^{2}, \kappa\right)$ s.t.

$$
\frac{1}{2} \int_{0}^{2 \pi}\left|\partial_{\theta} \varphi_{n}\left(x_{i}^{n}+r \mathrm{e}^{\imath \theta}\right)\right|^{2} \mathrm{~d} \theta \leq \pi+\frac{1}{\sqrt{|\ln \kappa|}} \text { for } i=1, \ldots, d
$$

This estimate is obtained via a mean value argument. We first prove that

$$
\mu_{\varepsilon_{n}}\left(\mathscr{R}\left(x_{i}^{n}, \kappa, \kappa^{2}\right), 1\right)=\mu_{\mathbb{I}}\left(\mathscr{R}\left(x_{i}^{n}, \kappa, \kappa^{2}\right), 1\right)+o_{\varepsilon_{n}}(1) .
$$

Indeed we let $\omega^{\prime}$ be a smooth open set s.t. $\bar{\omega} \subset \omega^{\prime}$ and $\overline{\omega^{\prime}} \subset B(0,1)$. We define $\alpha_{\varepsilon}^{\prime}=$ $\left\{\begin{array}{ll}b^{2} & \text { in } \delta \mathbb{Z} \times \delta \mathbb{Z}+\lambda \delta \cdot \omega^{\prime} \\ 1 & \text { otherwise }\end{array}\right.$. From Proposition 3, we have $\alpha_{\varepsilon}^{\prime} \leq U_{\varepsilon}^{2}+V_{\varepsilon}$ with $\left\|V_{\varepsilon}\right\|_{L^{\infty}}=$ $\mathcal{O}\left(\varepsilon^{2}\right)$ 
For $\rho \geq \delta$ and $x \in \mathbb{R}^{2}$, from Lemma [56, we have $\mathscr{H}^{1}\left[\left\{\alpha_{\varepsilon}^{\prime}=b^{2}\right\} \cap \partial B(x, \rho)\right] \leq 16 \pi^{2} \lambda \rho$. Therefore, using Lemma 53 we obtain

$$
\begin{aligned}
\mu_{\mathbb{I}}\left(\mathscr{R}\left(x_{i}^{n}, \kappa, \kappa^{2}\right), 1\right)+\mathcal{O}(\lambda|\ln \kappa|) \leq(\text { Lemma } 53) \leq & \mu_{\alpha_{\varepsilon_{n}}^{\prime}}\left(\mathscr{R}\left(x_{i}^{n}, \kappa, \kappa^{2}\right), 1\right) \\
\leq\left(\alpha_{\varepsilon}^{\prime} \leq U_{\varepsilon}^{2}+V_{\varepsilon}\right) \leq & \mu_{\varepsilon_{n}}\left(\mathscr{R}\left(x_{i}^{n}, \kappa, \kappa^{2}\right), 1\right)+o_{\varepsilon_{n}}(1) \\
\leq\left(U_{\varepsilon}^{2} \leq 1\right) \leq & \mu_{\mathbb{I}}\left(\mathscr{R}\left(x_{i}^{n}, \kappa, \kappa^{2}\right), 1\right)+o_{\varepsilon_{n}}(1) .
\end{aligned}
$$

Since for $s \in\left(\kappa^{2}, \kappa\right)$ we have $s \geq \delta$, we obtain (because $\kappa \geq \lambda$ )

$$
\mu_{\varepsilon_{n}}\left(\mathscr{R}\left(x_{i}^{n}, \kappa, \kappa^{2}\right), 1\right)=\mu_{\mathbb{I}}\left(\mathscr{R}\left(x_{i}^{n}, \kappa, \kappa^{2}\right), 1\right)+\mathcal{O}(\lambda|\ln \kappa|)=\pi|\ln \kappa|+o_{\varepsilon_{n}}(1) .
$$

Therefore from Corollary 14 and Lemma [52]2, $\frac{1}{2} \int_{\mathscr{R}\left(x_{i}^{n}, \kappa, \kappa^{2}\right)} U_{\varepsilon_{n}}^{2}\left|\nabla w_{n}\right|^{2}=\pi|\ln \kappa|+\mathcal{O}(1)$.

On the other hand, from a standard estimate, we have

$$
\frac{1}{2} \int_{0}^{2 \pi}\left|\partial_{\theta} \varphi_{n}\left(x_{i}^{n}+s \mathrm{e}^{\imath \theta}\right)\right|^{2} \mathrm{~d} \theta \geq \pi, \forall s \in\left(\kappa^{2}, \kappa\right) .
$$

We deduce that

$$
\pi d|\ln \kappa|+\mathcal{O}(1) \geq \frac{1}{2} \int_{\cup \mathscr{R}\left(x_{i}^{n}, \kappa, \kappa^{2}\right)}\left|\nabla w_{n}\right|^{2} \geq \frac{1}{2} \int_{\kappa^{2}}^{\kappa} \frac{\mathrm{d} s}{s} \sum_{i} \int_{0}^{2 \pi}\left|\partial_{\theta} \varphi_{n}\left(x_{i}^{n}+s \mathrm{e}^{\imath \theta}\right)\right|^{2} \mathrm{~d} \theta .
$$

Assume that $r \in\left(\kappa^{2}, \kappa\right)$ s.t. (E.2) holds does not exist. Then we obtain that for $s \in\left(\kappa^{2}, \kappa\right)$

$$
\sum_{i} \frac{1}{2} \int_{0}^{2 \pi}\left|\partial_{\theta} \varphi_{n}\left(x_{i}^{n}+s \mathrm{e}^{\imath \theta}\right)\right|^{2} \mathrm{~d} \theta>\pi d+\frac{1}{\sqrt{|\ln \kappa|}}
$$

and consequently

$$
\frac{1}{2} \int_{\cup \mathscr{R}\left(x_{i}^{n}, \kappa, \kappa^{2}\right)}\left|\nabla w_{n}\right|^{2} \geq|\ln \kappa|\left(\pi d+\frac{1}{\sqrt{|\ln \kappa|}}\right)=\pi d|\ln \kappa|+\sqrt{|\ln \kappa|} .
$$

Clearly this lower bound contradicts $\frac{1}{2} \int_{\mathscr{R}\left(x_{i}^{n}, \kappa, \kappa^{2}\right)} U_{\varepsilon_{n}}^{2}\left|\nabla w_{n}\right|^{2}=\pi|\ln \kappa|+\mathcal{O}(1)$. We are now in position to estimate the energy in $\mathscr{R}\left(x_{i}^{n}, \rho_{0}, r\right)$. Let $h_{i}^{n}: \mathbb{S}^{1} \rightarrow \mathbb{S}^{1}, h_{i}^{n}\left(\mathrm{e}^{\imath \theta}\right)=$ $w_{n}\left(x_{i}^{n}+r \mathrm{e}^{\imath \theta}\right)$. We have $h_{i}^{n} \times \partial_{\tau}\left[h_{i}^{n}\left(\mathrm{e}^{\imath \theta}\right)\right]=\partial_{\tau}\left[\varphi_{n}\left(x_{i}^{n}+r \mathrm{e}^{\imath \theta}\right)\right]$.

Thus from (E.2): $\left\|h_{i}^{n} \times \partial_{\tau} h_{i}^{n}\right\|_{L^{2}\left(\mathbb{S}^{1}\right)}^{2} \leq 2 \pi+2 / \sqrt{|\ln \kappa|}$. Consequently

$$
\int_{\mathbb{S}^{1}}\left|h_{i}^{n} \times \partial_{\tau} h_{i}^{n}-1\right|^{2}=\int_{\mathbb{S}^{1}}\left\{\left|h_{i}^{n} \times \partial_{\tau} h_{i}^{n}\right|^{2}+1-2 h_{i}^{n} \times \partial_{\tau} h_{i}^{n}\right\} \leq 2 / \sqrt{|\ln \kappa|} \rightarrow 0 .
$$

Therefore $h_{i}^{n} \times \partial_{\tau} h_{i}^{n} \rightarrow 1$ in $L^{2}\left(\mathbb{S}^{1}\right)$. Consequently, up to pass to a subsequence, we have the existence of $\alpha_{i} \in \mathbb{S}^{1}$ s.t. $\alpha_{i}^{-1} h_{i}^{n} \mathrm{e}^{-\imath \theta} \rightarrow 1$ in $H^{1}\left(\mathbb{S}^{1}\right)$.

From Propositions 12 and 13 in [13] we have

$$
\begin{aligned}
& \inf _{\substack{w \in H^{1}\left(\mathscr{R}\left(x_{i}^{n}, \rho_{0}, r\right), \mathbb{S}^{1}\right) \\
w\left(x_{i}^{n}+\rho_{0} \mathrm{e}^{\theta \theta}\right)=\alpha_{i} \mathrm{e}^{\theta \theta} \\
w\left(x_{i}^{n}+r \mathrm{e}^{\theta \theta}\right)=h_{i}^{n}\left(\mathrm{e}^{2 \theta}\right)}} \frac{1}{2} \int_{\mathscr{R}\left(x_{i}^{n}, \rho_{0}, r\right)}|\nabla w|^{2}=\inf _{\begin{array}{c}
w \in H^{1}\left(\mathscr{R}\left(x_{i}^{n}, \rho_{0}, r\right), \mathbb{S}^{1}\right) \\
w\left(x_{i}^{n}+\rho_{0} \mathrm{e}^{2 \theta}\right)=\alpha_{i} \mathrm{e}^{\mathrm{e}^{\theta}} \\
w\left(x_{i}^{n}+r \mathrm{e}^{\theta \theta}\right)=\alpha_{i} \mathrm{e}^{2 \theta}
\end{array}} \frac{1}{2} \int_{\mathscr{R}\left(x_{i}^{n}, \rho_{0}, r\right)}|\nabla w|^{2}+o_{n}(1) \\
& =\pi \ln \frac{\rho_{0}}{r}+o_{n}(1) \text {. }
\end{aligned}
$$


Step 3. We conclude

We are going to construct a map $\tilde{w}_{n} \in \mathcal{J}_{\rho}\left(\mathbf{y}_{n}\right), \max \left|y_{i}-b_{i}\right| \leq \delta$ and s.t.

$$
\int_{\Omega \backslash \cup \overline{B\left(y_{i}, \rho\right)}} U_{\varepsilon_{n}}^{2}\left|\nabla \tilde{w}_{n}\right|^{2}+\eta_{0} \leq \hat{\mathcal{J}}_{\rho, \varepsilon_{n}}\left(\mathbf{x}_{n}\right) .
$$

Clearly (E.3) is in contradiction with the assumption: $J_{\rho, \varepsilon_{n}}-\hat{\mathcal{J}}_{\rho, \varepsilon_{n}}\left(\mathbf{x}_{n}\right) \rightarrow 0$. Then this contradiction will imply that $\mathbf{a}=\lim \mathbf{x}_{\mathbf{n}}$ minimizes $W_{g}$.

We let $\mathbf{y}_{n}$ be s.t. $\max \left|y_{i}^{n}-b_{i}\right| \leq \delta$ and $x_{i}^{n}-y_{i}^{n} \in \delta \mathbb{Z} \times \delta \mathbb{Z}$ and we define

$$
\tilde{w}_{n}(x)= \begin{cases}w_{\rho_{0}}^{\mathbf{y}_{n}}(x) & \text { if } x \in \Omega \backslash \cup \overline{B\left(y_{i}^{n}, \rho_{0}\right)} \\ \operatorname{Cst}_{i, n} w^{i}\left(x-y_{i}^{n}+x_{i}^{n}\right) & \text { if } x \in \mathscr{R}\left(y_{i}^{n}, \rho_{0}, r\right) \\ \mathrm{Cst}_{i, n} w_{n}\left[x-y_{i}^{n}+x_{i}^{n}\right] & \text { if } x \in \mathscr{R}\left(y_{i}^{n}, r, \rho\right)\end{cases}
$$

Here:

- $w_{\rho_{0}}^{\mathbf{y}_{n}}$ is a minimizer of $\hat{\mathcal{J}}_{\rho_{0}, \mathbb{I}}\left(\mathbf{y}_{n}\right)$,

- $w^{i}$ is a minimizer of $\inf _{\begin{array}{c}w \in H^{1}\left(\mathscr{R}\left(x_{i}^{n}, \rho_{0}, r\right), \mathbb{S}^{1}\right) \\ w\left(x_{i}^{n}+\rho_{0} \mathrm{e}^{\imath \theta}\right)=\alpha_{i} \mathrm{e}^{\imath \theta} \\ w\left(x_{i}^{n}+r \mathrm{e}^{\imath \theta}\right)=h_{i}^{n}\left(\mathrm{e}^{\imath \theta}\right)\end{array}} \frac{1}{2} \int_{\mathscr{R}\left(x_{i}^{n}, \rho_{0}, r\right)}|\nabla w|^{2}$

- Cst $_{i, n} \in \mathbb{S}^{1}$ is a constant s.t. $\tilde{w}_{n} \in H^{1}\left(\Omega \backslash \cup \overline{B\left(y_{i}^{n}, \rho\right)}, \mathbb{S}^{1}\right)$

- $w_{n}$ is the minimizer of $\hat{\mathcal{J}}_{\rho, \varepsilon_{n}}\left(\mathbf{x}_{n}\right)$ used in Step 2 ..

We now compare the energies of $\tilde{w}_{n}$ and $w_{n}$.

$$
\begin{array}{r}
\int_{\Omega \backslash \cup \overline{B\left(y_{i}^{n}, \rho\right)}} U_{\varepsilon_{n}}^{2}\left|\nabla \tilde{w}_{n}\right|^{2}=\int_{\Omega \backslash \cup \overline{B\left(y_{i}^{n}, \rho_{0}\right)}} U_{\varepsilon_{n}}^{2}\left|\nabla \tilde{w}_{n}\right|^{2}+\int_{\cup_{i} \mathscr{R}\left(y_{i}^{n}, \rho_{0}, r\right)} U_{\varepsilon_{n}}^{2}\left|\nabla \tilde{w}_{n}\right|^{2}+ \\
+\int_{\cup_{i} \mathscr{R}\left(y_{i}^{n}, r, \rho\right)} U_{\varepsilon_{n}}^{2}\left|\nabla \tilde{w}_{n}\right|^{2} .
\end{array}
$$

From Step 1. (the definition of $\rho_{0}$ and Estimate (E.1) ), we have

$$
\begin{aligned}
\frac{1}{2} \int_{\Omega \backslash \cup \overline{B\left(y_{i}^{n}, \rho_{0}\right)}} U_{\varepsilon_{n}}^{2}\left|\nabla \tilde{w}_{n}\right|^{2} & \leq \pi d\left|\ln \rho_{0}\right|+W_{g}\left(\mathbf{y}_{n}\right)+\eta_{0}+o_{n}(1) \\
& \leq \pi d\left|\ln \rho_{0}\right|+W_{g}\left(\mathbf{x}_{n}\right)-10 \eta_{0} \\
& \leq \frac{1}{2} \int_{\Omega \backslash \cup \overline{B\left(x_{i}^{n}, \rho_{0}\right)}} U_{\varepsilon_{n}}^{2}\left|\nabla w_{n}\right|^{2}-2 \eta_{0}
\end{aligned}
$$

From Step 2., letting $\alpha_{\varepsilon}^{\prime}=\left\{\begin{array}{ll}b^{2} & \text { in } \delta \mathbb{Z} \times \delta \mathbb{Z}+\lambda \delta \cdot \omega^{\prime} \\ 1 & \text { otherwise }\end{array}\right.$, we have

$$
\begin{aligned}
& \frac{1}{2} \int_{\cup_{i} \mathscr{R}\left(y_{i}^{n}, \rho_{0}, r\right)} U_{\varepsilon_{n}}^{2}\left|\nabla \tilde{w}_{n}\right|^{2}=(\text { Step 2. })=\quad \pi d \ln \frac{\rho_{0}}{r}+o_{n}(1) \\
& \leq\left(\text { Lem. } \left[53 \&[56) \leq \frac{1}{2} \int_{\cup_{i} \mathscr{R}\left(x_{i}^{n}, \rho_{0}, r\right)} \alpha^{\prime}\left|\nabla w_{n}\right|^{2}+o_{n}(1)\right.\right. \\
& \leq\left(\alpha_{\varepsilon}^{\prime} \leq U_{\varepsilon}^{2}+V_{\varepsilon}\right) \leq \frac{1}{2} \int_{\cup_{i} \mathscr{R}\left(x_{i}^{n}, \rho_{0}, r\right)} U_{\varepsilon_{n}}^{2}\left|\nabla w_{n}\right|^{2}+o_{n}(1) .
\end{aligned}
$$


From Lemma 47

$$
\int_{\cup_{i} \mathscr{R}\left(y_{i}^{n}, r, \rho\right)} U_{\varepsilon_{n}}^{2}\left|\nabla \tilde{w}_{n}\right|^{2}=\int_{\cup_{i} \mathscr{R}\left(x_{i}^{n}, r, \rho\right)} U_{\varepsilon_{n}}^{2}\left|\nabla w_{n}\right|^{2}+o_{n}(1) .
$$

Therefore we obtain (E.3) and consequently Proposition 16 holds.

\section{E.3 Proof of Proposition 17}

The strategy to prove Proposition 17 is the following:

Step 1. We let $\kappa=\max (\lambda, \delta)$. We first characterize almost minimal configurations for $I_{\kappa, \varepsilon}$ (i.e the domain $\Omega$ is perforated by discs with radius $\kappa$ ).

Step 2. We make the description of almost minimal points $\left(x_{\varepsilon}\right)_{\varepsilon}$ for $\mu_{\varepsilon}\left(\mathscr{R}\left(\cdot, \kappa, \lambda \delta^{3 / 2}\right), 1\right)$.

Step 3. We estimate $\inf _{x_{0} \in \mathbb{R}^{2}} \mu_{\varepsilon}\left(\mathscr{R}\left(x_{0}, \lambda \delta^{3 / 2}, \rho\right), 1\right)$ and we conclude.

Step 1. We study almost minimal configurations for $I_{\kappa, \varepsilon}, \kappa=\max (\lambda, \delta)$

We prove that $\{\mathbf{x}, \mathbf{d}\}=\left\{\left(x_{1}^{\varepsilon}, d_{1}\right), \ldots,\left(x_{N}^{\varepsilon}, d_{N}\right)\right\}$ is an almost minimal configuration for $I_{\kappa, \varepsilon}$ if and only if $N=d, d_{i}=1$ and there is $\eta_{0}>0$ s.t. $\operatorname{dist}\left(x_{i}^{\varepsilon}, \partial \Omega\right),\left|x_{i}^{\varepsilon}-x_{j}^{\varepsilon}\right| \geq \eta_{0}$. easily

First note that for $\eta_{0}>0$ and $x_{1}^{\varepsilon}, \ldots, x_{d}^{\varepsilon} \in \Omega$ s.t. $\operatorname{dist}\left(x_{i}, \partial \Omega\right),\left|x_{i}^{\varepsilon}-x_{j}^{\varepsilon}\right| \geq \eta_{0}$ we have

$$
I_{\kappa, \varepsilon} \leq \hat{\mathcal{I}}_{\kappa, \varepsilon}(\mathbf{x}, \mathbf{d}) \leq \pi d|\ln \kappa|+C\left(\eta_{0}\right)
$$

with $C\left(\eta_{0}\right)$ which is independent of $\varepsilon$.

We consider $\{\mathbf{x}, \mathbf{d}\}$ which is almost minimal for $I_{\kappa, \varepsilon}$. We argue as in the proof of Proposition 12 (Assertions 1 and 2, see Subsections D.3 \& D.4). We use the separation process defined Subsection C.1 and the associated natural partition of $\Omega_{\kappa}:=\Omega \backslash \cup \overline{B\left(x_{i}^{\varepsilon}, \kappa\right)}$ (see Subsection C.2).

Here the key ingredients are Lemmas 53 \& 56 (which replace the periodic structure of the pinning term). Combining both lemmas we get that if $R>r \geq \kappa$, then

$$
\mu_{\varepsilon}\left(\mathscr{R}\left(x_{0}, R, r\right), 1\right)=\pi \ln \frac{R}{r}+\mathcal{O}\left(\lambda \ln \frac{R}{r}\right) .
$$

The rings $\mathscr{R}\left(x_{0}, R, r\right)$ which occur in the partition of $\Omega_{\kappa}$ are all s.t. $C(\Omega) \geq R>$ $r \geq \kappa$ and thus $\frac{R}{r}=\mathcal{O}\left(\kappa^{-1}\right)$. Which infer that $\mathcal{O}\left(\lambda \ln \frac{R}{r}\right)=o_{\varepsilon}(1)$ and consequently $\mu_{\varepsilon}\left(\mathscr{R}\left(x_{0}, R, r\right), 1\right)=\pi \ln \frac{R}{r}+o_{\varepsilon}(1)$

Therefore we get: If $\{\mathbf{x}, \mathbf{d}\}$ is an almost minimal configuration for $I_{\kappa, \varepsilon}$ then $N=d$, $d_{i}=1$ and there is $\eta_{0}>0$ s.t. $\operatorname{dist}\left(x_{i}^{\varepsilon}, \partial \Omega\right),\left|x_{i}^{\varepsilon}-x_{j}^{\varepsilon}\right| \geq \eta_{0}$. This is proved by contradiction exactly as in Subsections D.3 \& D.4 and using (E.4).

Moreover, if $\{\mathbf{x}, \mathbf{d}\}$ is an almost minimal configuration for $I_{\kappa, \varepsilon}$, then the arguments of Subsections D.3 \& D.4 in conjunction with (E.4), yield $\left|\hat{\mathcal{I}}_{\kappa, \varepsilon}(\mathbf{x}, \mathbf{d})-\pi d\right| \ln \kappa \| \leq C\left(\eta_{0}\right)$. Here $\eta_{0}$ is obtained in the previous paragraph. Therefore we get $I_{\kappa, \varepsilon}=\pi d|\ln \kappa|+\mathcal{O}(1)$.

Conversely, from (E.4), for $\eta_{0}>0$ and $x_{1}^{\varepsilon}, \ldots, x_{d}^{\varepsilon} \in \Omega$ s.t. $\operatorname{dist}\left(x_{i}^{\varepsilon}, \partial \Omega\right),\left|x_{i}^{\varepsilon}-x_{j}^{\varepsilon}\right| \geq \eta_{0}$, we have $\left\{x_{1}^{\varepsilon}, \ldots, x_{d}^{\varepsilon}\right\}$ which is almost minimal for $I_{\kappa, \varepsilon}$.

Step 2. We study almost minimal configurations for $\mu_{\varepsilon}\left(\mathscr{R}\left(\cdot, \kappa, \lambda \delta^{3 / 2}\right), 1\right)$

For $j \in\{1, \ldots, P\}$, we denote: $\omega_{\varepsilon}^{j}:=\cup_{i \in \mathcal{M}_{j}^{\varepsilon}}\left\{y_{i, j}^{\varepsilon}+\lambda \delta^{j} \omega\right\}$. And recall that the set of centers of connected components of $\omega_{\varepsilon}^{j}$ is $\widehat{\mathcal{M}}_{j}^{\varepsilon}:=\left\{y_{i, j}^{\varepsilon} \mid i \in \mathcal{M}_{j}^{\varepsilon}\right\}$. 
Letting $x_{\varepsilon}^{0} \in \omega_{\varepsilon}$ et $c>0$ (independent of $\varepsilon$ ) s.t. $B\left(x_{\varepsilon}^{0}, c \lambda \delta\right) \subset \omega_{\varepsilon}^{1}$, on the one hand we may easily prove that

$$
\mu_{\varepsilon}\left(\mathscr{R}\left(x_{\varepsilon}^{0}, \delta, \lambda \delta^{3 / 2}\right), 1\right)=\pi b^{2}\left|\ln \delta^{1 / 2}\right|+\pi|\ln \lambda|+\mathcal{O}(1),
$$

and on the other hand, applying Lemmas 53 \& 56, we have

$$
\mu_{\varepsilon}\left(\mathscr{R}\left(x_{\varepsilon}^{0}, \kappa, \delta\right), 1\right)=\pi[1+\mathcal{O}(\lambda)] \ln \frac{\kappa}{\delta} .
$$

Therefore, from (E.5) and (E.6), we get

$$
\mu_{\varepsilon}\left(\mathscr{R}\left(x_{\varepsilon}^{0}, \kappa, \lambda \delta^{3 / 2}\right), 1\right)=\pi\left[\frac{b^{2}}{2}+1+\mathcal{O}(\lambda)\right]|\ln \delta|+\pi \ln \frac{\kappa}{\lambda}+\mathcal{O}(1) .
$$

We are going to prove that this situation $\left(B\left(x_{\varepsilon}^{0}, c \lambda \delta\right) \subset \omega_{\varepsilon}^{1}\right)$ is the only way to get the minimal energy. More precisely we prove that for a fixed constant $C_{0}>0$, if we have $\left(x_{\varepsilon}\right)_{\varepsilon} \subset \Omega$ which is s.t.

$$
\mu_{\varepsilon}\left(\mathscr{R}\left(x_{\varepsilon}, \kappa, \lambda \delta^{3 / 2}\right), 1\right) \leq \inf _{x_{0} \in \Omega} \mu_{\varepsilon}\left(\mathscr{R}\left(x_{0}, \kappa, \lambda \delta^{3 / 2}\right), 1\right)+C_{0},
$$

then there is $c>0$ independent of $\varepsilon$ s.t. for sufficiently small $\varepsilon$ we have $B\left(x_{\varepsilon}, c \lambda \delta\right) \subset \omega_{\varepsilon}$, i.e. $B\left(x_{\varepsilon}, c \lambda \delta\right) \subset y_{i_{\varepsilon}, 1}^{\varepsilon}+\lambda \delta \omega$ with $y_{i_{\varepsilon}, 1}^{\varepsilon} \in \widehat{\mathcal{M}}_{1}^{\varepsilon}$.

We let $C_{0}>0$ and $\left(x_{\varepsilon}\right)_{\varepsilon} \subset \Omega$ s.t. (E.8) holds.

Up to pass to a sequence $\varepsilon_{n} \downarrow 0$, dropping the subscript $n$ (we write $\varepsilon$ instead of $\varepsilon_{n}$ ), we may assume that one of these cases occurs

Case 0. $\exists c>0$ s.t. $B\left(x_{\varepsilon}, c \lambda \delta\right) \subset \omega_{\varepsilon}$,

Case 1. $x_{\varepsilon} \notin \cup_{j=1}^{P} \cup_{i \in \mathcal{M}_{j}^{\varepsilon}} B\left(y_{i, j}^{\varepsilon}, \delta^{j}\right)$,

Case 2. $x_{\varepsilon} \in \cup_{j=2}^{P} \cup_{i \in \mathcal{M}_{j}^{\varepsilon}} B\left(y_{i, j}^{\varepsilon}, \delta^{j}\right)$,

Case 3. $\left\{x_{\varepsilon} \in \cup_{i \in \mathcal{M}_{1}^{\varepsilon}} B\left(y_{i, 1}^{\varepsilon}, \delta\right) \backslash \overline{\omega_{\varepsilon}^{1}}\right\}$ or $\left\{x_{\varepsilon} \in \omega_{\varepsilon}^{1} \& \operatorname{dist}\left(x_{\varepsilon}, \partial \omega_{\varepsilon}^{1}\right) / \lambda \delta \rightarrow 0\right\}$.

We want to prove that only Case 0. occurs if (E.8) holds.

Case 1. From Lemmas $53 \&$ 56, it is direct to prove that

$$
\mu_{\varepsilon}\left(\mathscr{R}\left(x_{\varepsilon}, \kappa, \lambda \delta^{3 / 2}\right), 1\right) \geq \pi[1+\mathcal{O}(\lambda)] \ln \frac{\kappa}{\lambda \delta^{3 / 2}}=\pi\left[\frac{3}{2}+\mathcal{O}(\lambda)\right]|\ln \delta|+\pi \ln \frac{\kappa}{\lambda} .
$$

Using (E.7) we get

$$
\mu_{\varepsilon}\left(\mathscr{R}\left(x_{\varepsilon}, \kappa, \lambda \delta^{3 / 2}\right), 1\right)-\inf _{x_{0} \in \Omega} \mu_{\varepsilon}\left(\mathscr{R}\left(x_{0}, \kappa, \lambda \delta^{3 / 2}\right), 1\right) \rightarrow+\infty .
$$

Therefore, if $\left(x_{\varepsilon}\right)_{\varepsilon}$ satisfies (E.8), then Case 1. does not occur.

Case 2. We let $j_{0} \in\{2, \ldots, P\}$ be s.t. $x_{\varepsilon} \in \cup_{i \in \mathcal{M}_{j_{0}}^{\varepsilon}} B\left(y_{i, j_{0}}, \delta^{j_{0}}\right)$. We define $\kappa^{\prime}:=$ $\max \left\{\delta^{j_{0}}, \lambda \delta^{3 / 2}\right\}$ and we denote $y_{0}=y_{i, j_{0}}^{\varepsilon} \in \widehat{\mathcal{M}}_{j_{0}}$ be s.t. $x_{\varepsilon} \in B\left(y_{0}, \delta^{j_{0}}\right)$.

We first assume that $x_{\varepsilon} \notin \overline{\omega_{\varepsilon}}$ and we let $æ=\max \left\{\lambda \delta^{3 / 2}\right.$, dist $\left.\left(x_{\varepsilon}, \partial \omega_{\varepsilon}^{j_{0}}\right)-\lambda \delta^{j_{0}}\right\}$. In order to estimate $\mu_{\varepsilon}\left(\mathscr{R}\left(x_{\varepsilon}, \kappa, \lambda \delta^{3 / 2}\right), 1\right)$, we divide $\mathscr{R}\left(x_{\varepsilon}, \kappa, \lambda \delta^{3 / 2}\right)$ into

$$
\mathscr{R}\left(x_{\varepsilon}, \kappa, \kappa^{\prime}+2 \lambda \delta^{j_{0}}\right) \cup \mathscr{R}\left(x_{\varepsilon}, \kappa^{\prime}+2 \lambda \delta^{j_{0}}, æ+2 \lambda \delta^{j_{0}}\right) \cup \mathscr{R}\left(x_{\varepsilon}, æ+2 \lambda \delta^{j_{0}}, æ\right) \cup \mathscr{R}\left(x_{\varepsilon}, æ, \lambda \delta^{3 / 2}\right) .
$$


From Lemmas 53 \& 56 we have

$$
\mu_{\varepsilon}\left(\mathscr{R}\left(x_{\varepsilon}, \kappa, \kappa^{\prime}+2 \lambda \delta^{j_{0}}\right), 1\right) \geq \pi[1+\mathcal{O}(\lambda)] \ln \frac{\kappa}{\kappa^{\prime}+2 \lambda \delta^{j_{0}}} .
$$

Note that $\operatorname{dist}\left(\mathscr{R}\left(x_{\varepsilon}, \kappa^{\prime}+2 \lambda \delta^{j_{0}}, æ+2 \lambda \delta^{j_{0}}\right), \omega_{\varepsilon}^{j_{0}}\right) \geq \lambda \delta^{j_{0}}$ and if for some $j$ we have $\mathscr{R}\left(x_{\varepsilon}, \kappa^{\prime}+\right.$ $\left.2 \lambda \delta^{j_{0}}, æ+2 \lambda \delta^{j_{0}}\right) \cap \omega_{\varepsilon}^{j} \neq \emptyset$, then $\operatorname{dist}\left(x_{\varepsilon}, \omega_{\varepsilon}^{j}\right) \geq \delta^{j}$ (because $x_{\varepsilon} \in B\left(y_{0}, \delta^{j_{0}}\right)$ ). Therefore, using Proposition 3 and Lemmas 53 \& 56 we get

$$
\mu_{\varepsilon}\left(\mathscr{R}\left(x_{\varepsilon}, \kappa^{\prime}+2 \lambda \delta^{j_{0}}, æ+2 \lambda \delta^{j_{0}}\right), 1\right) \geq \pi[1+\mathcal{O}(\lambda)] \ln \frac{\kappa^{\prime}+2 \lambda \delta^{j_{0}}}{æ+2 \lambda \delta^{j_{0}}} .
$$

It is obvious that

$$
\mu_{\varepsilon}\left(\mathscr{R}\left(x_{\varepsilon}, æ+2 \lambda \delta^{j_{0}}, æ\right), 1\right) \geq b^{2} \pi \ln \frac{æ+2 \lambda \delta^{j_{0}}}{æ} \geq b^{2} \pi \ln \left(1+2 \delta^{j_{0}-3 / 2}\right)=o_{\varepsilon}(1) .
$$

By definition of $æ$, from Proposition 3, we have

$$
\mu_{\varepsilon}\left(\mathscr{R}\left(x_{\varepsilon}, æ, \lambda \delta^{3 / 2}\right), 1\right) \geq \pi \ln \frac{æ}{\lambda \delta^{3 / 2}}-o_{\varepsilon}(1) .
$$

Summing these lower bounds we have

$$
\begin{aligned}
\mu_{\varepsilon}\left(\mathscr{R}\left(x_{\varepsilon}, \kappa, \lambda \delta^{3 / 2}\right), 1\right) & \geq \pi[1+\mathcal{O}(\lambda)]\left[\ln \frac{\kappa}{\kappa^{\prime}+2 \lambda \delta^{j_{0}}}+\ln \frac{\kappa^{\prime}+2 \lambda \delta^{j_{0}}}{æ+2 \lambda \delta^{j_{0}}}+\ln \frac{æ}{\lambda \delta^{3 / 2}}\right]+o_{\varepsilon}(1) \\
& \geq \pi[1+\mathcal{O}(\lambda)] \ln \frac{\kappa}{\lambda \delta^{3 / 2}}+o_{\varepsilon}(1) \\
& =\pi[1+\mathcal{O}(\lambda)]\left(\frac{3}{2}|\ln \delta|+\ln \frac{\kappa}{\lambda}\right)+o_{\varepsilon}(1)
\end{aligned}
$$

and therefore $\mu_{\varepsilon}\left(\mathscr{R}\left(x_{\varepsilon}, \kappa, \lambda \delta^{3 / 2}\right), 1\right)-\inf _{x_{0} \in \Omega} \mu_{\varepsilon}\left(\mathscr{R}\left(x_{0}, \kappa, \lambda \delta^{3 / 2}\right), 1\right) \rightarrow+\infty$ (because $0 \leq$ $\ln (\kappa / \lambda) \leq|\ln \delta|$ and from (E.7) $)$.

We now assume that $x_{\varepsilon} \in \omega_{\varepsilon}$. Because $j_{0} \geq 2$ and $x_{\varepsilon} \in B\left(y_{0}, \lambda \delta^{j_{0}}\right)$, we have $B\left(y_{0}, 2 \lambda \delta^{j_{0}}\right) \cap \mathscr{R}\left(x_{\varepsilon}, \kappa, \lambda \delta^{3 / 2}\right)=\emptyset$. Therefore, from the dilution of the inclusion, if there is $\tilde{\omega}_{\varepsilon}$, a connected component of $\omega_{\varepsilon}^{j}$ s.t. $\mathscr{R}\left(x_{\varepsilon}, \kappa, \lambda \delta^{3 / 2}\right) \cap \tilde{\omega}_{\varepsilon}$, then $\operatorname{dist}\left(x_{\varepsilon}, \tilde{\omega}_{\varepsilon}\right) \geq \delta^{j} / 3$. Consequently, from Lemmas 53 \& 56, we have

$$
\mu_{\varepsilon}\left(\mathscr{R}\left(x_{\varepsilon}, \kappa, \lambda \delta^{3 / 2}\right), 1\right) \geq \pi[1+\mathcal{O}(\lambda)] \ln \frac{\kappa}{\lambda \delta^{3 / 2}}=\pi[1+\mathcal{O}(\lambda)]\left(\frac{3}{2}|\ln \delta|+\ln \frac{\kappa}{\lambda}\right) .
$$

From (E.7), we obtain that $\mu_{\varepsilon}\left(\mathscr{R}\left(x_{\varepsilon}, \kappa, \lambda \delta^{3 / 2}\right), 1\right)-\inf _{x_{0} \in \Omega} \mu_{\varepsilon}\left(\mathscr{R}\left(x_{0}, \kappa, \lambda \delta^{3 / 2}\right), 1\right) \rightarrow+\infty$.

We deduce that if $\left(x_{\varepsilon}\right)_{\varepsilon}$ satisfies (E.8), then Case 2. does not occur.

Case 3. We denote $y_{0}:=y_{i, 1}^{\varepsilon} \in \widehat{\mathcal{M}}_{1}^{\varepsilon}$ be s.t. $x_{\varepsilon} \in B\left(y_{0}, \delta\right)$.

On the one hand, if $\kappa \leq 10^{-2} \delta$, then we have $\mu_{\varepsilon}\left(\mathscr{R}\left(x_{\varepsilon}, \kappa, \delta\right), 1\right), \mu_{\varepsilon}\left(\mathscr{R}\left(y_{0}, \kappa, \delta\right), 1\right) \leq$ $2 \pi \ln 10$.

On the other hand, if $\kappa>10^{-2} \delta$, then we have $\mathscr{R}\left(y_{0}, \kappa, 10 \delta\right) \subset \mathscr{R}\left(x_{\varepsilon}, 10 \kappa, 10^{-1} \delta\right)$ and thus (using Proposition 45) we get

$$
\begin{aligned}
\mu_{\varepsilon}\left(\mathscr{R}\left(x_{\varepsilon}, \kappa, \delta\right), 1\right) & \geq \mu_{\varepsilon}\left(\mathscr{R}\left(x_{\varepsilon}, 10 \kappa, 10^{-1} \delta\right), 1\right)-\left(2 \pi \ln 10+C_{b}\right) \\
& \geq \mu_{\varepsilon}\left(\mathscr{R}\left(y_{0}, \kappa, 10 \delta\right), 1\right)-\left(2 \pi \ln 10+C_{b}\right) \\
& \geq \mu_{\varepsilon}\left(\mathscr{R}\left(y_{0}, \kappa, 10 \delta\right), 1\right)-\left(3 \pi \ln 10+2 C_{b}\right) .
\end{aligned}
$$


Moreover, following the argument of Subsection D.5, we have (because $\frac{\lambda \delta^{3 / 2}}{\lambda \delta} \rightarrow 0$ )

$$
\mu_{\varepsilon}\left(\mathscr{R}\left(x_{\varepsilon}, \delta, \lambda \delta^{3 / 2}\right), 1\right)-\mu_{\varepsilon}\left(\mathscr{R}\left(y_{0}, \delta, \lambda \delta^{3 / 2}\right), 1\right) \rightarrow+\infty .
$$

Therefore we have the existence of $H_{\varepsilon} \rightarrow+\infty$ as $\varepsilon \rightarrow 0$ s.t.

$$
\begin{aligned}
\mu_{\varepsilon}\left(\mathscr{R}\left(x_{\varepsilon}, \kappa, \lambda \delta^{3 / 2}\right), 1\right) & \geq \mu_{\varepsilon}\left(\mathscr{R}\left(x_{\varepsilon}, \kappa, \delta\right), 1\right)+\mu_{\varepsilon}\left(\mathscr{R}\left(x_{\varepsilon}, \delta, \lambda \delta^{3 / 2}\right), 1\right) \\
& \geq \mu_{\varepsilon}\left(\mathscr{R}\left(y_{0}, \kappa, \delta\right), 1\right)+\mu_{\varepsilon}\left(\mathscr{R}\left(y_{0}, \delta, \lambda \delta^{3 / 2}\right), 1\right)+H_{\varepsilon} \\
\text { (Prop. 46.3) } & \geq \mu_{\varepsilon}\left(\mathscr{R}\left(y_{0}, \kappa, \lambda \delta^{3 / 2}\right), 1\right)+H_{\varepsilon}-2 C_{b} .
\end{aligned}
$$

Consequently $\mu_{\varepsilon}\left(\mathscr{R}\left(x_{\varepsilon}, \kappa, \lambda \delta^{3 / 2}\right), 1\right)-\inf _{x_{0} \in \Omega} \mu_{\varepsilon}\left(\mathscr{R}\left(x_{0}, \kappa, \lambda \delta^{3 / 2}\right), 1\right) \rightarrow+\infty$ and since $\left(x_{\varepsilon}\right)_{\varepsilon}$ satisfies (E.8), Case 3 does not occur.

Step 3. We study $\inf _{x_{0} \in \mathbb{R}^{2}} \mu_{\varepsilon}\left(\mathscr{R}\left(x_{0}, \lambda \delta^{3 / 2}, \rho\right), 1\right)$ and we conclude

It is obvious that $\inf _{x_{0} \in \mathbb{R}^{2}} \mu_{\varepsilon}\left(\mathscr{R}\left(x_{0}, \lambda \delta^{3 / 2}, \rho\right), 1\right)=\pi b^{2} \ln \frac{\lambda \delta^{3 / 2}}{\rho}+o_{\varepsilon}(1)$. Now we are in position to conclude. On the one hand, from the previous steps, for $\eta_{0}, c>0$ and a configuration of points/degrees $\left\{\mathbf{x}_{\varepsilon}, \mathbf{1}\right\}=\left\{\left(x_{1}^{\varepsilon}, 1\right), \ldots,\left(x_{d}^{\varepsilon}, 1\right)\right\}$ s.t. $\left|x_{i}^{\varepsilon}-x_{j}^{\varepsilon}\right|$, $\operatorname{dist}\left(x_{i}^{\varepsilon}, \partial \Omega\right) \geq$ $\eta_{0}$ and $B\left(x_{i}^{\rho, \varepsilon}, c \lambda \delta\right) \subset \omega_{\varepsilon}^{1}$ for all $i \neq j, i, j \in\{1, \ldots, N\}$, we have $\hat{\mathcal{I}}_{\rho, \varepsilon}\left(\mathbf{x}_{\varepsilon}\right)=I_{\rho, \varepsilon}+\mathcal{O}(1)$.

On the other hand, for $\varepsilon_{n} \downarrow 0$, if either there is $i \in\{1, \ldots, N\}$ s.t. $d_{i}>1$ or $\operatorname{dist}\left(x_{i}^{\rho, \varepsilon}, \partial \Omega\right) \rightarrow 0$ or there are $i \neq j$ s.t. $\left|x_{i}-x_{j}\right| \rightarrow 0$, then the configuration of points/degrees cannot be almost minimal for $I_{\delta, \varepsilon_{n}}$ and thus it cannot be almost minimal for $I_{\rho, \varepsilon_{n}}$.

Moreover, if there is $i$ s.t. $x_{i}^{\varepsilon_{n}} \notin \omega_{\varepsilon_{n}}^{1}$ or $\operatorname{dist}\left(x_{i}^{\varepsilon_{n}}, \partial \omega_{\varepsilon_{n}}^{1}\right) /(\lambda \delta) \rightarrow 0$, then $\left(x_{i}^{\varepsilon_{n}}\right)_{n}$ cannot be an almost minimal configuration for $\mu_{\varepsilon}\left(\mathscr{R}\left(\cdot, \kappa, \lambda \delta^{3 / 2}\right), 1\right)$. And thus $\{\mathbf{x}, \mathbf{d}\}$ cannot be an almost minimal configuration for $I_{\rho, \varepsilon_{n}}$.

Therefore Assertions 1. and 2. of Proposition 17 holds.

The rest of the proposition is obtained exactly as Corollary 14

\section{F Proof of Proposition 32}

We use the unfolding operator (see [8], definition 2.1). We define, for $\Omega_{0} \subset \mathbb{R}^{2}$ an open set, $p \in(1, \infty)$ and $\delta>0$ :

$$
\begin{aligned}
\mathcal{T}_{\delta}: L^{p}\left(\Omega_{0}\right) & \rightarrow \\
\phi & \mapsto \mathcal{T}_{\delta}(\phi)(x, y)=\left\{\begin{array}{cl}
L^{p}\left(\Omega_{0} \times \tilde{Y}\right) \\
\phi\left(\delta\left[\frac{x}{\delta}\right]+\delta y\right) & \text { for }(x, y) \in \tilde{\Omega}_{\delta}^{\text {incl }} \times \tilde{Y} \\
0 & \text { for }(x, y) \in \Lambda_{\delta} \times \tilde{Y}
\end{array}\right.
\end{aligned}
$$

and

$$
\begin{aligned}
& \tilde{Y}=(0,1) \times(0,1), \quad \tilde{\Omega}_{\delta}^{\text {incl }}:=\bigcup_{\tilde{Y}_{\delta}^{K} \subset \Omega_{0}} \overline{\tilde{Y}_{\delta}^{K}}, \\
& \tilde{Y}_{\delta}^{K}=\delta(K+\tilde{Y}), K \in \mathbb{Z}^{2} \\
& \Lambda_{\delta}:=\Omega_{0} \backslash \tilde{\Omega}_{\delta}^{\text {incl }} \quad \text { and } \quad\left[\frac{x}{\delta}\right]:=\left(\left[\frac{x_{1}}{\delta}\right],\left[\frac{x_{2}}{\delta}\right]\right) \in \mathbb{Z}^{2} .
\end{aligned}
$$

Here, for $s \in \mathbb{R},[s]$ is the integer part of $s$.

We will use the following results:

$\mathcal{T}_{\delta}$ is linear and continuous, of norm at most 1 ([8], Proposition 2.5), 


$$
\begin{gathered}
\mathcal{T}_{\delta}(\phi \psi)=\mathcal{T}_{\delta}(\phi) \mathcal{T}_{\delta}(\psi)([8] \text {, equation }(2.2)) \\
\left.\delta \mathcal{T}_{\delta}(\nabla \phi)(x, y)=\nabla_{y} \mathcal{T}_{\delta}(\phi)(x, y) \text { for } \phi \in W^{1, p}\left(\Omega_{0}\right) \text { ([8], equation }(3.1)\right),
\end{gathered}
$$

for $\phi \in L^{1}\left(\Omega_{0}\right)$, we have $\int_{\tilde{\Omega}_{\delta}^{\text {incl }}} \phi=\int_{\Omega_{0} \times \tilde{Y}} \mathcal{T}_{\delta}\left(\phi_{\delta}\right)$ ([8], Proposition. 2.5 (i)).

If $\phi_{\delta} \in H^{1}\left(\Omega_{0}\right)$ is such that $\phi_{\delta} \rightarrow \phi_{0}$ in $H^{1}$, then, up to subsequence, there exists $\hat{\phi} \in$ $L^{2}\left(\Omega_{0}, H_{\mathrm{per}}^{1}(\tilde{Y})\right)$ s.t.:

$$
\mathcal{T}_{\delta}\left(\phi_{\delta}\right) \rightarrow \phi_{0} \text { and } \mathcal{T}_{\delta}\left(\nabla \phi_{\delta}\right) \rightarrow \nabla \phi_{0}+\nabla_{y} \hat{\phi} \text { in } L^{2}\left(\Omega_{0} \times \tilde{Y}\right)([8] \text {, Theorem 3.5). }
$$

Here $H_{\text {per }}^{1}(\tilde{Y})$ stands for the set of functions $\phi \in H^{1}(\tilde{Y})$ s.t. the extending of $\phi$ by $\tilde{Y}$ periodicity is in $H_{\mathrm{loc}}^{1}\left(\mathbb{R}^{2}\right)$ (see [9], section 3.4).

In order to define properly the homogenized matrix $\mathcal{A}$ we recall a classical result (see Theorem 4.27 in [9]).

Proposition 58. Let $H_{0} \in L^{\infty}\left(\tilde{Y},\left[b^{2}, 1\right]\right)$. For all $f \in\left(H_{\mathrm{per}}^{1}(\tilde{Y})\right)^{\prime}$ s.t. $f$ annihilates the constants there exists a unique solution $h \in H_{\mathrm{per}}^{1}(\tilde{Y})$ of

$$
\operatorname{div}\left(H_{0} \nabla_{y} h\right)=f \text { and } \mathcal{M}_{\tilde{Y}}(h)=\int_{\tilde{Y}} h=0
$$

Using the previous theorem we denote $\chi_{j} \in H_{\text {per }}^{1}(\tilde{Y})$ the unique solution of

$$
\operatorname{div}\left(H_{0} \nabla_{y} \chi_{j}\right)=\partial_{y_{j}}\left(H_{0}\right) \text { and } \mathcal{M}_{\tilde{Y}}\left(\chi_{j}\right)=0 .
$$

With these auxiliary functions, we can give an explicit expression of $\mathcal{A}$ the homogenized matrix of $H_{0}(\dot{\bar{\delta}}) \operatorname{Id}_{\mathbb{R}^{2}}$ (see Theorem 6.1 in [9]):

$$
\mathcal{A}=\int_{\tilde{Y}} H_{0}\left(\begin{array}{cc}
1-\partial_{y_{1}} \chi_{1} & -\partial_{y_{1}} \chi_{2} \\
-\partial_{y_{2}} \chi_{1} & 1-\partial_{y_{2}} \chi_{2}
\end{array}\right)=\int_{\tilde{Y}} H_{0}\left(\operatorname{Id}_{\mathbb{R}^{2}}-\nabla_{y} \chi\right), \chi=\left(\chi_{1}, \chi_{2}\right) .
$$

For the convenience of the reader we restate, in larger detail, Proposition 32 ,

Proposition. Let $\Omega_{0} \subset \mathbb{R}^{2}$ be a smooth bounded open set and let $v_{n} \in H^{2}\left(\Omega_{0}, \mathbb{C}\right)$ be s.t.

1. $\left|v_{n}\right| \leq 1$ and $\int_{\Omega_{0}}\left(1-\left|v_{n}\right|^{2}\right)^{2} \rightarrow 0$,

2. $v_{n} \rightarrow v_{*}$ in $H^{1}\left(\Omega_{0}\right)$ and $v_{*} \in H^{1}\left(\Omega_{0}, \mathbb{S}^{1}\right)$,

3. there is $H_{n} \in W^{1, \infty}\left(\Omega_{0},\left[b^{2}, 1\right]\right)$ and $\delta_{n} \downarrow 0$ s.t. $\mathcal{T}_{\delta_{n}}\left(H_{n}\right) \rightarrow H_{0}$ in $L^{2}\left(\Omega_{0} \times \tilde{Y}\right)$ with $H_{0}$ independent of $x \in \Omega_{0}$,

$$
\text { 4. }-\operatorname{div}\left(H_{n} \nabla v_{n}\right)=v_{n} f_{n}(x), f_{n} \in L^{\infty}\left(\Omega_{0}, \mathbb{R}\right) .
$$

Then $v_{*}$ is a solution of

$$
-\operatorname{div}\left(\mathcal{A} \nabla v_{*}\right)=\left(\mathcal{A} \nabla v_{*} \cdot \nabla v_{*}\right) v_{*} .
$$

Here $\mathcal{A}$ is the homogenized matrix of $H_{0}(\dot{\bar{\delta}}) \operatorname{Id}_{\mathbb{R}^{2}}$ given by

$$
\mathcal{A}=\int_{\tilde{Y}} H_{0}\left(\begin{array}{cc}
1-\partial_{y_{1}} \chi_{1} & -\partial_{y_{1}} \chi_{2} \\
-\partial_{y_{2}} \chi_{1} & 1-\partial_{y_{2}} \chi_{2}
\end{array}\right) \text {. }
$$


Proof. In order to keep notations simple, we write, in what follows, $\delta$ rather than $\delta_{n}$.

Since $f_{n}$ is real valued, we have that $\operatorname{div}\left(H_{n} \nabla v_{n}\right) \times v_{n}=0$. From (F.1) and (F.2), we obtain

$$
\operatorname{div}_{y}\left[\mathcal{T}_{\delta}\left(H_{n}\right)(x, y) \mathcal{T}_{\delta}\left(\nabla v_{n}\right)(x, y)\right] \times \mathcal{T}_{\delta}\left(v_{n}\right)(x, y)=0 \text { in } \Omega_{0} \times \tilde{Y}
$$

Note that from the assumptions and (F.1), (F.5), passing to a subsequence, there is $\hat{w} \in$ $L^{2}\left(\Omega_{0}, H_{\text {per }}^{1}(\tilde{Y})\right)$ s.t.

$$
\mathcal{T}_{\delta}\left(v_{n}\right)(x, y) \rightarrow v_{*}(x), \mathcal{T}_{\delta}\left(\nabla v_{n}\right)(x, y) \rightarrow \nabla v_{*}(x)+\nabla_{y} \hat{v}(x, y) \text { in } L^{2}\left(\Omega_{0} \times \tilde{Y}\right)
$$

and

$$
\mathcal{T}_{\delta}\left(H_{n}\right)(x, y) \rightarrow H_{0}(y) \text { in } L^{2}\left(\Omega_{0} \times \tilde{Y}\right) .
$$

Thus we obtain the convergence:

$\operatorname{div}_{y}\left[\mathcal{T}_{\delta}\left(H_{n}\right)(x, y) \mathcal{T}_{\delta}\left(\nabla v_{n}\right)(x, y)\right] \times \mathcal{T}_{\delta}\left(v_{n}\right)(x, y) \rightarrow \operatorname{div}_{y}\left[H_{0}\left(\nabla v_{*}+\nabla_{y} \hat{v}\right)\right] \times v_{*}$ in $L^{2}\left(\Omega_{0} \times H^{-1}(\tilde{Y})\right)$.

Consequently,

$$
\operatorname{div}_{y}\left[H_{0}\left(\nabla v_{*}+\nabla_{y} \hat{v}\right)\right] \times v_{*}=0 .
$$

Since $v_{*}$ is independent of $y \in \tilde{Y}$, the previous assertion is equivalent to

$$
-\operatorname{div}_{y}\left[H_{0} \nabla_{y}\left(\hat{v} \times v_{*}\right)\right]=\left(\nabla_{y} H_{0} \cdot \nabla v_{*}\right) \times v_{*},
$$

which in turn is equivalent to

$$
-\operatorname{div}_{y}\left[H_{0} \nabla_{y}\left(\hat{v} \times v_{*}\right)\right]=\sum_{i} \partial_{y_{i}} H_{0}\left(\partial_{i} v_{*} \times v_{*}\right) .
$$

Hence, from Proposition 58 and (F.6), we obtain

$$
\hat{v} \times v_{*}=-\sum_{i} \chi_{i}\left(\partial_{i} v_{*} \times v_{*}\right)=-\chi \cdot\left(\nabla v_{*} \times v_{*}\right), \chi=\left(\chi_{1}, \chi_{2}\right) .
$$

Let $\psi \in \mathcal{D}\left(\Omega_{0}\right)$ and $n$ sufficiently large s.t. $\operatorname{Supp}(\psi) \subset \tilde{\Omega}_{\delta}^{\text {incl }}$. Since $-\operatorname{div}\left[H_{n} \nabla v_{n} \times v_{n}\right]=0$, we have

$$
\int_{\tilde{\Omega}_{\delta}^{\text {incl }}} H_{n} \nabla v_{n} \times v_{n} \cdot \nabla \psi=0 .
$$

This identity combined with (F.4) implies that

$$
\int_{\Omega_{0} \times \tilde{Y}} \mathcal{T}_{\delta}\left[H_{n}\left(\nabla v_{n} \times v_{n}\right) \cdot \nabla \psi\right]=0 .
$$

Therefore, using ( $(\underline{\text { F.3 }})$ and $(\underline{\text { F.5 }})$, we obtain:

$$
\begin{aligned}
0=\int_{\Omega_{0} \times \tilde{Y}} \mathcal{T}_{\delta}\left[H_{n}\left(\nabla v_{n} \times v_{n}\right) \cdot \nabla \psi\right] & =\int_{\Omega_{0} \times \tilde{Y}} \mathcal{T}_{\delta}\left(H_{n}\right) \mathcal{T}_{\delta}\left(\nabla v_{n}\right) \times \mathcal{T}_{\delta}\left(v_{n}\right) \cdot \mathcal{T}_{\delta}(\nabla \psi) \\
& \rightarrow{ }_{n \rightarrow \infty} \int_{\Omega_{0} \times \tilde{Y}} H_{0}\left[\nabla v_{*} \times v_{*}+\nabla_{y}\left(\hat{v} \times v_{*}\right)\right] \cdot \nabla \psi
\end{aligned}
$$

Finally, for all $\psi \in \mathcal{D}\left(\Omega_{0}\right)$, using $(\overline{\mathrm{F} .8})$, we have

$$
\begin{aligned}
0=\int_{\Omega_{0} \times \tilde{Y}} H_{0} \nabla v_{*} \times v_{*}\left[\operatorname{Id}_{\mathbb{R}^{2}}-\nabla_{y} \chi\right] \cdot \nabla \psi & =\int_{\Omega_{0}}\left(\left\{\int_{\tilde{Y}} H_{0}\left[\operatorname{Id}_{\mathbb{R}^{2}}-\nabla_{y} \chi\right]\right\} \nabla v_{*} \times v_{*}\right) \nabla \psi \\
& =-\int_{\Omega_{0}}-\operatorname{div}\left(\mathcal{A} \nabla v_{*} \times v_{*}\right) \psi .
\end{aligned}
$$


Here $\mathcal{A}=\int_{\tilde{Y}} H_{0}\left(\operatorname{Id}_{\mathbb{R}^{2}}-\nabla_{y} \chi\right)$.

Thus $-\operatorname{div}\left(\mathcal{A} \nabla v_{*} \times v_{*}\right)=0$. Note that, since $H_{0}$ and $\chi$ are independent of $x, \mathcal{A}$ is a constant matrix. This fact combined with the equation $-\operatorname{div}\left(\mathcal{A} \nabla v_{*} \times v_{*}\right)=0$ implies that $v_{*}$ satisfies

$$
-\operatorname{div}\left(\mathcal{A} \nabla v_{*}\right)=\left(\mathcal{A} \nabla v_{*} \cdot \nabla v_{*}\right) v_{*} .
$$

Indeed, we can always consider $\varphi_{*}$ which is locally defined in $\Omega_{0}$ and whose gradient is globally defined and in $L^{2}\left(\Omega_{0}, \mathbb{R}^{2}\right)$ s.t. $v_{*}=\mathrm{e}^{\imath \varphi_{*}}$.

Since $v_{*} \times \nabla v_{*}=\nabla \varphi_{*}$ we obtain that $\operatorname{div}\left(\mathcal{A} \nabla \varphi_{*}\right)=0$. Identity (F.9) follows from the equation of $\varphi_{*}$ and the fact that $\left|\nabla \varphi_{*}\right|^{2}=\left|\nabla v_{*}\right|^{2}$.

\section{References}

[1] A. Aftalion, E. Sandier, and S. Serfaty. Pinning Phenomena in the Ginzburg-Landau model of Superconductivity. J. Math. Pures Appl., 80(3):339-372, 2001.

[2] P. Bauman, N. Carlson, and D. Phillips. On the zeros of solutions to Ginzburg-Landau type systems. SIAM J. Math. Anal., 24(5):1283-1293, 1993.

[3] F. Bethuel, H. Brezis, and F. Hélein. Asymptotics for the minimization of a GinzburgLandau functional. Calc. Var. Partial Differential Equations, 1(2):123-148, 1993.

[4] F. Bethuel, H. Brezis, and F. Hélein. Ginzburg-Landau Vortices. Progress in Nonlinear Differential Equations and their Applications, 13. Birkhäuser Boston Inc., Boston, MA, 1994.

[5] F. Bethuel, G. Orlandi, and D. Smets. Improved estimates for the Ginzburg-Landau equation: the elliptic case. Ann. Sc. Norm. Super. Pisa Cl. Sci. (5), 4(2):319-355, 2005.

[6] H. Brezis. Équations de Ginzburg-Landau et singularités. Notes de cours rédigées par Vicenţiu Rădulescu. http://inf.ucv.ro/radulescu/articles/coursHB.pdf, 2001.

[7] H. Brezis, F. Merle, and T. Rivière. Quantization effects for $-\Delta u=u\left(1-|u|^{2}\right)$ in $\mathbb{R}^{2}$. Arch. Rational Mech. Anal., 126(1):35-58, 1994.

[8] D. Cioranescu, A. Damlamian, and G. Griso. The periodic unfolding method in homogenization. SIAM J. Math. Anal., 40(4):1585-1620, 2008.

[9] D. Cioranescu and P. Donato. An Introduction to Homogenization, volume 17 of Oxford Lecture Series in Mathematics and its Applications. The Clarendon Press Oxford University Press, 1999.

[10] M. Comte and P. Mironescu. The behavior of a ginzburg- landau minimizer near its zeroes. Calc. Var. Partial Differential Equations, 4(4):323-340, 1996.

[11] M. Dos Santos. Défauts de vorticité dans un supraconducteur en présence d'impuretés. PhD thesis, Univ. Lyon 1, 2010. 
[12] M. Dos Santos, P. Mironescu, and O. Misiats. The Ginzburg-Landau functional with a discontinuous and rapidly oscillating pinning term. Part I: the zero degree case. Commun. Contemp. Math., 13(5):1-30, 2011.

[13] M. Dos Santos and O. Misiats. Ginzburg-Landau model with small pinning domains. Netw. Heterog. Media, 6(4), 2011.

[14] Z. C. Han and I. Shafrir. Lower bounds for the energy of $S^{1}$-valued maps in perforated domains. J. Anal. Math., 66:295-305, 1995.

[15] A. Kachmar. Magnetic vortices for a Ginzburg-Landau type energy with discontinuous constraint. ESAIM: Control, Optimisation and Calculus of Variations, 16(3):545-580, 2009 .

[16] L. Lassoued. Sur quelques équations aux dérivées partielles non linéaires issues de la géométrie et de la physique. PhD thesis, Université de Paris 06 Paris, 1996.

[17] L. Lassoued and P. Mironescu. Ginzburg-landau type energy with discontinuous constraint. J. Anal. Math., 77:1-26, 1999.

[18] P. Mironescu. Explicit bounds for solutions to a Ginzburg-Landau type equation. Rev. Roumaine Math. Pures Appl., 41(3-4):263-271, 1996.

[19] P. Mironescu. Les minimiseurs locaux pour l'équation de Ginzburg-Landau sont à symétrie radiale. C. R. Acad. Sci. Paris Sér. I Math., 323:593-598, 1996.

[20] E. Sandier and S. Serfaty. Vortices in the Magnetic Ginzburg-Landau Model. Birkhäuser Boston Inc., Boston, MA, 2007.

[21] M. Sauvageot. Periodic Unfolding Method and Homogenization for the GinzburgLandau Equation. arXiv:0904.1828v1, 2009.

[22] I. Shafrir. Remarks on solutions of $-\Delta u=\left(1-|u|^{2}\right) u$ in $\mathbb{R}^{2}$. C. R. Acad. Sci. Paris Sér. I Math., 318(4):327-331, 1994.

[23] M. Tinkham. Introduction to Superconductivity. McGraw-Hill, New York, 1996. 